Supporting Information for

\title{
Novel and efficient Synthesis of Iminocoumarins via Copper-Catalyzed Multicoponent Reaction
}

\author{
Sun-Liang Cui, Xu-Feng Lin, Yan-Guang Wang* \\ Department of Chemistry, Zhejiang University, Hangzhou 310027, P.R. China
}

\section{Table of Contents}

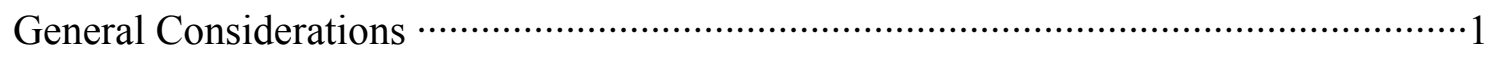

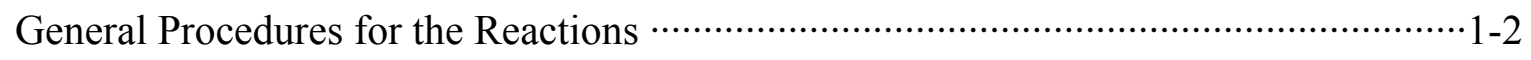

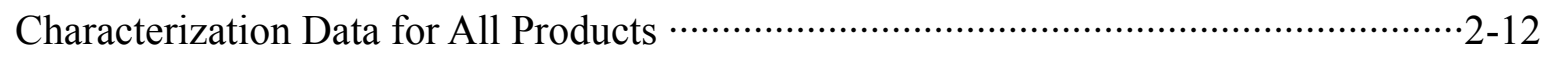

References and Notes

Copies of ${ }^{1} \mathrm{H}$ and ${ }^{13} \mathrm{C}$ NMR Spectra for All Products ….......................................14-58 


\section{General Considerations}

Column chromatography was carried out on silica gel (300-400 mesh) with mixed solvents (hexane-ethyl acetate). THF and triethyl amine (TEA) were distilled from Sodium and $\mathrm{CaH}_{2}$ respectively. Infrared spectra were obtained on a FTIR spectrometer. NMR spectra were recorded for ${ }^{1} \mathrm{H}$ NMR at $400 \mathrm{MHz}$ otherwise noted, for ${ }^{13} \mathrm{C} \mathrm{NMR}$ at $100 \mathrm{MHz}$ at $293 \mathrm{~K}$ otherwise noted. Chemical shifts are reported relative to residue peaks of $\mathrm{CDCl}_{3}$ (7.27 ppm for ${ }^{1} \mathrm{H}$ and $77.27 \mathrm{ppm}$ for ${ }^{13} \mathrm{C}$ ) and DMSO-d6 (2.50 ppm for ${ }^{1} \mathrm{H}$ and $40.0 \mathrm{ppm}$ for $\left.{ }^{13} \mathrm{C}\right)$. The following abbreviations are used to describe peak patterns where appropriate: $b=$ broad, $s=$ singlet, $\mathrm{d}=$ doublet, $\mathrm{t}=$ triplet, $\mathrm{q}=$ quartet, $\mathrm{m}=$ multiplet. Coupling constants are reported in Hertz (Hz). Low-resolution MS and HRMS were obtained using ESI ionization. Melting points were measured with micro melting point apparatus. Sulfonyl azides were prepared according the published methods. ${ }^{1,2}$

\section{General Procedures for the Reaction}

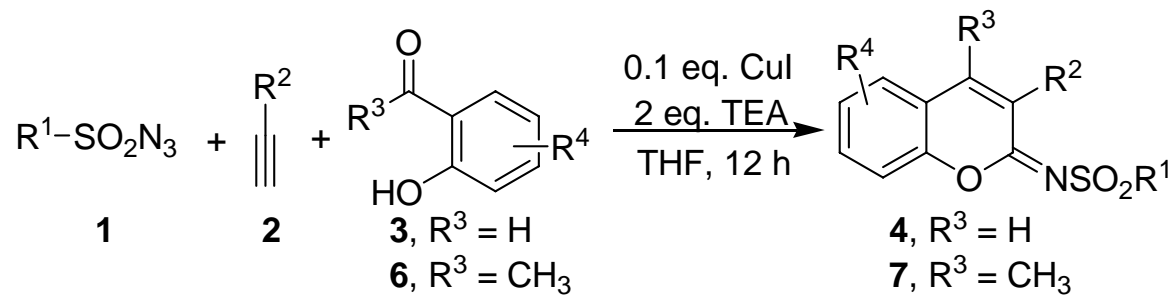

To a solution of sulfonyl azide ( $1 \mathrm{mmol})$, alkynes $(1 \mathrm{mmol}), \mathrm{CuI}(0.1 \mathrm{mmo})$ and salicylaldehde or o-hydroxyacetophenone $(1.1 \mathrm{mmol})$ in THF $(3 \mathrm{~mL})$ in Schlenk tube was added TEA (2 mmol) slowly via syringe. The reaction solution was stirred at room temperature under $\mathrm{N}_{2}$ for $12 \mathrm{~h}$. The solution was concentrated, and the residue was diluted with $\mathrm{CH}_{2} \mathrm{Cl}_{2}(30 \mathrm{~mL})$, washed with water $(10 \mathrm{~mL})$, brine $(10 \mathrm{~mL})$ and dried over sodium sulfate. The solvent was removed in vacuum, and the residue was purified by column 
chromatography on silica gel with hexane-ethyl acetate $(2: 1)$ to give corresponding iminocoumarin. All the compounds were further recrystallized from EA.

\section{Characterization Data for All Products}

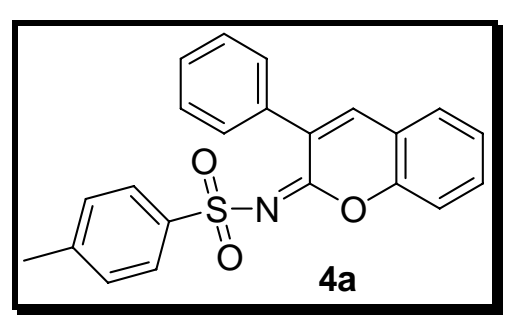

Pale yellow solid; mp. 194-195 ${ }^{\circ} \mathrm{C} ;{ }^{1} \mathrm{H}$ NMR $\left(\mathrm{CDCl}_{3}\right) \delta 7.94$ (d, $\left.J=8.4 \mathrm{~Hz}, 2 \mathrm{H}\right), 7.68(\mathrm{~s}, 1 \mathrm{H})$, $7.54(\mathrm{~m}, 4 \mathrm{H}), 7.42(\mathrm{~d}, J=8.0 \mathrm{~Hz}, 1 \mathrm{H}), 7.34(\mathrm{~m}, 6 \mathrm{H}), 2.38(\mathrm{~s}, 3 \mathrm{H}) \mathrm{ppm} ;{ }^{13} \mathrm{C} \mathrm{NMR}\left(\mathrm{CDCl}_{3}\right) \delta$ $157.6,152.4,143.2,139.9,139.4,134.5,132.1,130.1,129.7,129.4,129.2,129.1,128.43$, 128.40, 127.4, 126.6, 125.9, 119.9, 116.4, 21.7 ppm; IR (KBr) v 3065, 1635, 1628, 1558, 1305, 1182, 1088, $863 \mathrm{~cm}^{-1}$; MS (ESI) m/z $397.8\left([\mathrm{M}+\mathrm{Na}]^{+}\right)$; HRMS (ESI) calcd for $\mathrm{C}_{22} \mathrm{H}_{17} \mathrm{NO}_{3} \mathrm{~S}\left([\mathrm{M}+\mathrm{Na}]^{+}\right), 398.0821$; found, 398.0803.

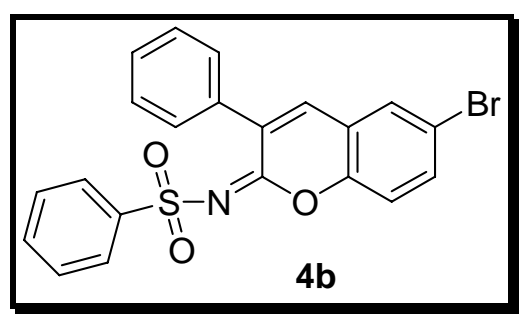

Pale yellow solid; mp. 202-204 ${ }^{\circ} \mathrm{C} ;{ }^{1} \mathrm{H}$ NMR $\left(\mathrm{CDCl}_{3}\right) \delta 8.03(\mathrm{~m}, 2 \mathrm{H}), 7.67-7.44(\mathrm{~m}, 8 \mathrm{H}), 7.37$ $(\mathrm{m}, 4 \mathrm{H}) \mathrm{ppm} ;{ }^{13} \mathrm{C} \mathrm{NMR}\left(\mathrm{CDCl}_{3}\right) \delta 157.1,151.3,142.2,138.3,134.8,134.1,132.7,131.5$ $130.6,129.6,129.3,128.9,128.5,127.2,121.6,118.7,118.4$ ppm; IR (KBr) v 3066, 1617, 15560, 1545, 1320, 1149, $818 \mathrm{~cm}^{-1}$; MS (ESI) m/z $461.8\left([\mathrm{M}+\mathrm{Na}]^{+}\right)$; HRMS (ESI) calcd for $\mathrm{C}_{21} \mathrm{H}_{14} \mathrm{BrNO}_{3} \mathrm{~S}\left([\mathrm{M}+\mathrm{Na}]^{+}\right), 461.9770$; found, 461.9750 . 


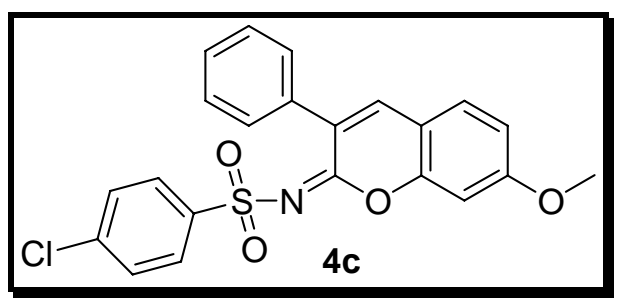

Pale yellow solid; mp. $261-265{ }^{\circ} \mathrm{C} ;{ }^{1} \mathrm{H}$ NMR (DMSO-d6) $\delta 8.20(\mathrm{~s}, 1 \mathrm{H}), 8.09$ (d, $J=8.4 \mathrm{~Hz}$, 2H), $7.76(\mathrm{~d}, J=8.4 \mathrm{~Hz}, 1 \mathrm{H}), 7.68(\mathrm{~d}, J=8.8 \mathrm{~Hz}, 2 \mathrm{H}), 7.61(\mathrm{~d}, J=6.8 \mathrm{~Hz}, 2 \mathrm{H}), 7.43(\mathrm{~m}, 3 \mathrm{H})$, $7.10\left(\mathrm{dd}, J_{1}=2.0 \mathrm{~Hz}, J_{2}=8.4 \mathrm{~Hz}, 1 \mathrm{H}\right), 7.03(\mathrm{~d}, J=2.0 \mathrm{~Hz}, 1 \mathrm{H}), 3.94(\mathrm{~s}, 3 \mathrm{H}) \mathrm{ppm} ;{ }^{13} \mathrm{C} \mathrm{NMR}$ (DMSO-d6) $\delta 163.5,158.3,153.8,142.6,141.1,138.0,135.0,130.8,129.8,129.60,129.57$, 128.9, 128.6, 125.2, 115.1, 113.9, 100.1, 56.8 ppm; IR (KBr) v 3088, 1635, 1578, 1558, 1288, 1087, 895, $767 \mathrm{~cm}^{-1}$; MS (ESI) m/z $447.8\left([\mathrm{M}+\mathrm{Na}]^{+}\right)$; HRMS (ESI) calcd for $\mathrm{C}_{22} \mathrm{H}_{16} \mathrm{ClNO}_{4} \mathrm{~S}$ $\left([\mathrm{M}+\mathrm{Na}]^{+}\right), 448.0381 ;$ found, 448.0381 .

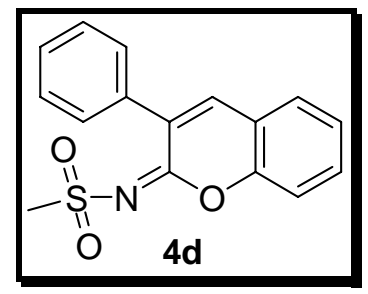

Pale yellow crystal; mp. 152-154 ${ }^{\circ} \mathrm{C} ;{ }^{1} \mathrm{H}$ NMR $\left(\mathrm{CDCl}_{3}\right) \delta 7.71(\mathrm{~s}, 1 \mathrm{H}), 7.59(\mathrm{~m}, 2 \mathrm{H}), 7.54(\mathrm{~m}$, 3H), $7.42(\mathrm{~m}, 3 \mathrm{H}), 7.34(\mathrm{~m}, 1 \mathrm{H}), 3.08(\mathrm{~s}, 3 \mathrm{H}) \mathrm{ppm} ;{ }^{13} \mathrm{C} \mathrm{NMR}\left(\mathrm{CDCl}_{3}\right) \delta 157.9,152.4,139.9$, 134.7, 132.1, 129.9, 129.2, 129.1, 128.4, 128.3, 125.9, 119.8, 116.9, $43.2 \mathrm{ppm}$; IR (KBr) $v$ $3032,2930,1624,1558,1550,1305,1139,954,767 \mathrm{~cm}^{-1} ; \mathrm{MS}(\mathrm{ESI}) \mathrm{m} / \mathrm{z} 321.8\left([\mathrm{M}+\mathrm{Na}]^{+}\right)$; HRMS (ESI) calcd for $\mathrm{C}_{16} \mathrm{H}_{13} \mathrm{NO}_{3} \mathrm{~S}\left([\mathrm{M}+\mathrm{Na}]^{+}\right)$, 322.0508; found, 322.0500.

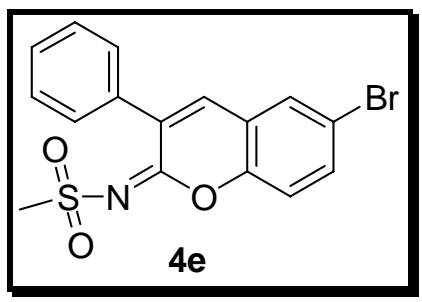


White crystal; mp. $214-216{ }^{\circ} \mathrm{C} ;{ }^{1} \mathrm{H}$ NMR $\left(\mathrm{CDCl}_{3}\right) \delta 7.66(\mathrm{~m}, 2 \mathrm{H}), 7.62(\mathrm{~s}, 1 \mathrm{H}), 7.59(\mathrm{~m}, 2 \mathrm{H})$, $7.45(\mathrm{~m}, 4 \mathrm{H}), 3.09(\mathrm{~s}, 3 \mathrm{H}) \mathrm{ppm} ;{ }^{13} \mathrm{C} \mathrm{NMR}\left(\mathrm{CDCl}_{3}\right) \delta 157.2,151.3,138.2,134.8,134.4,131.4$ $130.5,129.5,129.2,128.6,121.5,118.8,118.6,43.3$ ppm; IR (KBr) v 3050, 2930, 1621, 1576, 1560, 1304, 1130, $817 \mathrm{~cm}^{-1}$; MS (ESI) m/z $400.1\left([\mathrm{M}+\mathrm{Na}]^{+}\right)$; HRMS (ESI) calcd for $\mathrm{C}_{16} \mathrm{H}_{12} \mathrm{BrNO}_{3} \mathrm{~S}\left([\mathrm{M}+\mathrm{Na}]^{+}\right), 399.9613$; found, 399.9595.

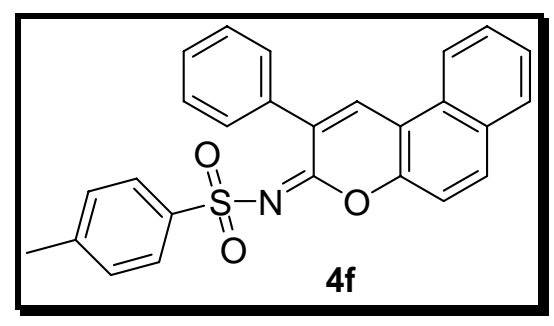

Green solid; mp. $213-217{ }^{\circ} \mathrm{C} ;{ }^{1} \mathrm{H}$ NMR $\left(\mathrm{CDCl}_{3}\right) \delta 8.40(\mathrm{~d}, J=4.0 \mathrm{~Hz}, 1 \mathrm{H}), 8.22(\mathrm{~m}, 1 \mathrm{H})$, $8.00(\mathrm{~d}, J=8.0 \mathrm{~Hz}, 2 \mathrm{H}), 7.97(\mathrm{~d}, J=3.6 \mathrm{~Hz}, 1 \mathrm{H}), 7.90(\mathrm{~d}, J=8.0 \mathrm{~Hz}, 1 \mathrm{H}), 7.70(\mathrm{~m}, 3 \mathrm{H}), 7.54$ $(\mathrm{m}, 2 \mathrm{H}), 7.42(\mathrm{~m}, 3 \mathrm{H}), 7.30(\mathrm{~d}, J=8.0 \mathrm{~Hz}, 2 \mathrm{H}), 2.40(\mathrm{~s}, 3 \mathrm{H}) \mathrm{ppm} ;{ }^{13} \mathrm{C} \mathrm{NMR}\left(\mathrm{CDCl}_{3}\right) \delta 157.6$, $152.1,143.2,139.7,135.8,134.9,133.5,130.9,129.42,129.38,129.3,128.8,128.7,128.5$ 127.4, 126.9, 121.7, 116.2, 114.6, 21.7 ppm; IR (KBr) v 3060, 1631, 1609, 1545, 1513, 1291, 1087, $870 \mathrm{~cm}^{-1}$; MS (ESI) $\mathrm{m} / \mathrm{z} 448.2\left([\mathrm{M}+\mathrm{Na}]^{+}\right)$; HRMS (ESI) calcd for $\mathrm{C}_{26} \mathrm{H}_{19} \mathrm{NO}_{3} \mathrm{~S}$ $\left([\mathrm{M}+\mathrm{Na}]^{+}\right), 448.0978 ;$ found, 448.0957.

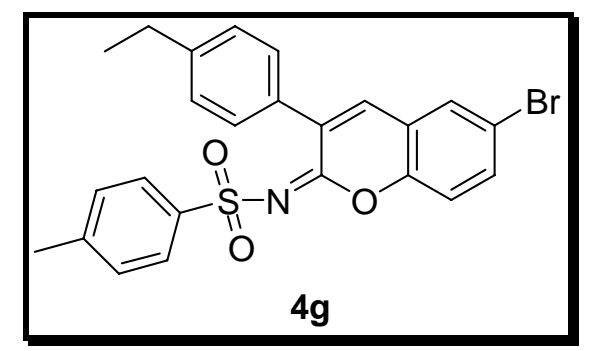

Pale yellow solid; mp. 175-178 ${ }^{\circ} \mathrm{C} ;{ }^{1} \mathrm{H}$ NMR $\left(\mathrm{CDCl}_{3}\right) \delta 7.94(\mathrm{~d}, J=8.4 \mathrm{~Hz}, 2 \mathrm{H}), 7.63(\mathrm{~m}$, 2H), $7.58(\mathrm{~s}, 1 \mathrm{H}), 7.52(\mathrm{~d}, J=8.0 \mathrm{~Hz}, 2 \mathrm{H}), 7.35(\mathrm{~d}, J=8.4 \mathrm{~Hz}, 1 \mathrm{H}), 7.29(\mathrm{~m}, 2 \mathrm{H}), 7.23(\mathrm{~d}, J$ $=7.6 \mathrm{~Hz}, 2 \mathrm{H}), 2.68(\mathrm{q}, J=7.6 \mathrm{~Hz}, 2 \mathrm{H}), 2.41(\mathrm{~s}, 3 \mathrm{H}), 1.26(\mathrm{t}, J=7.6 \mathrm{~Hz}, 3 \mathrm{H}) \mathrm{ppm} ;{ }^{13} \mathrm{C} \mathrm{NMR}$ 
$\left(\mathrm{CDCl}_{3}\right) \delta 157.0,151.2,146.0,143.4,139.5,137.5,134.6,131.6,131.5,130.5,129.5,129.3$ $128.1,127.5,121.7,118.6,118.3,28.9,21.8,15.6$ ppm; IR (KBr) v 2970, 1621, 1581, 1570, 1302, 1089, 937, 813, $683 \mathrm{~cm}^{-1}$; MS (ESI) m/z $504.2\left([\mathrm{M}+\mathrm{Na}]^{+}\right)$; HRMS (ESI) calcd for $\mathrm{C}_{24} \mathrm{H}_{20} \mathrm{BrNO}_{3} \mathrm{~S}\left([\mathrm{M}+\mathrm{Na}]^{+}\right), 504.0239$; found, 504.0216.

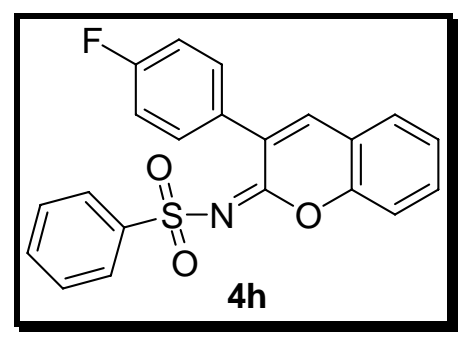

Green solid; mp. 201-204 ${ }^{\circ} \mathrm{C} ;{ }^{1} \mathrm{H}$ NMR $\left(\mathrm{CDCl}_{3}\right) \delta 8.06(\mathrm{~d}, J=7.2 \mathrm{~Hz}, 2 \mathrm{H}), 7.71(\mathrm{~s}, 1 \mathrm{H}), 7.54$ $(\mathrm{m}, 7 \mathrm{H}), 7.44(\mathrm{~d}, J=8.4 \mathrm{~Hz}, 1 \mathrm{H}), 7.35(\mathrm{t}, J=7.6 \mathrm{~Hz}, 1 \mathrm{H}), 7.07(\mathrm{t}, J=8.8 \mathrm{~Hz}, 2 \mathrm{H}) \mathrm{ppm} ;{ }^{13} \mathrm{C}$ $\operatorname{NMR}\left(\mathrm{CDCl}_{3}\right) \delta 163.3\left(\mathrm{~d}, J_{\mathrm{C}-\mathrm{F}}=247.2 \mathrm{~Hz}\right), 157.7,152.5,142.3,139.9,132.7,132.3,131.2(\mathrm{~d}$, $\left.J_{\mathrm{C}-\mathrm{F}}=8.3 \mathrm{~Hz}\right), 130.5\left(\mathrm{~d}, J_{\mathrm{C}-\mathrm{F}}=3.0 \mathrm{~Hz}\right), 129.2,128.9,128.4,127.3,126.1,119.9,116.6,115.5$ $\left(\mathrm{d}, J_{\mathrm{C}-\mathrm{F}}=21.5 \mathrm{~Hz}\right) \mathrm{ppm}$; IR $(\mathrm{KBr}) v 3100,1624,1585,1546,1323,1161,1090,758 \mathrm{~cm}^{-1}$; MS (ESI) $\mathrm{m} / \mathrm{z} 401.8\left([\mathrm{M}+\mathrm{Na}]^{+}\right)$; HRMS (ESI) calcd for $\mathrm{C}_{21} \mathrm{H}_{14} \mathrm{FNO}_{3} \mathrm{~S}\left([\mathrm{M}+\mathrm{Na}]^{+}\right), 402.0571$; found, 402.0560 .

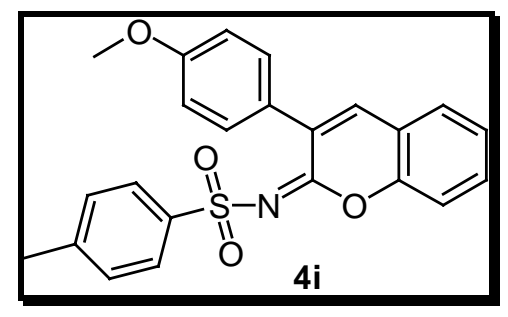

Yellow solid; mp. 173-174 ${ }^{\circ} \mathrm{C} ;{ }^{1} \mathrm{H}$ NMR $\left(\mathrm{CDCl}_{3}\right) \delta 7.80(\mathrm{~d}, J=8.4 \mathrm{~Hz}, 2 \mathrm{H}), 7.63(\mathrm{~s}, 1 \mathrm{H})$, $7.55(\mathrm{~d}, J=8.8 \mathrm{~Hz}, 2 \mathrm{H}), 7.49(\mathrm{~d}, J=8.0 \mathrm{~Hz}, 2 \mathrm{H}), 7.40(\mathrm{~d}, J=8.0 \mathrm{~Hz}, 1 \mathrm{H}), 7.29(\mathrm{~m}, 3 \mathrm{H}), 6.88$ $(\mathrm{d}, J=8.4 \mathrm{~Hz}, 2 \mathrm{H}), 3.80(\mathrm{~s}, 3 \mathrm{H}), 2.38(\mathrm{~s}, 3 \mathrm{H}) \mathrm{ppm} ;{ }^{13} \mathrm{C} \mathrm{NMR}\left(\mathrm{CDCl}_{3}\right) \delta 160.4,157.8,152.2$ $143.2,139.5,138.7,131.7,130.6,129.7,129.4,128.2,127.5,126.8,125.9,120.1,116.4$ 
113.9, 55.5, 21.7 ppm; IR $(\mathrm{KBr}) \vee 3034,2926,1624,1608,1540,1301,1152,1086,832 \mathrm{~cm}^{-1}$; MS (ESI) m/z $427.8\left([\mathrm{M}+\mathrm{Na}]^{+}\right)$; HRMS (ESI) calcd for $\mathrm{C}_{23} \mathrm{H}_{19} \mathrm{NO}_{4} \mathrm{~S}\left([\mathrm{M}+\mathrm{Na}]^{+}\right), 428.0927$; found, 428.0906 .

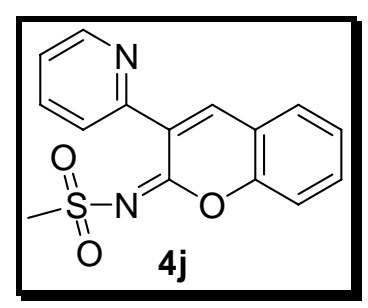

Brown solid; mp. $115-119{ }^{\circ} \mathrm{C} ;{ }^{1} \mathrm{H}$ NMR $\left(\mathrm{CDCl}_{3}\right) \delta 8.70(\mathrm{~s}, 1 \mathrm{H}), 8.60(\mathrm{~s}, 1 \mathrm{H}), 8.24(\mathrm{~d}, J=8.0$ $\mathrm{Hz}, 1 \mathrm{H}), 7.76(\mathrm{~m}, 1 \mathrm{H}), 7.60(\mathrm{~m}, 3 \mathrm{H}), 7.37(\mathrm{~m}, 1 \mathrm{H}), 7.31(\mathrm{~m}, 1 \mathrm{H}), 3.20(\mathrm{~s}, 3 \mathrm{H}) \mathrm{ppm} ;{ }^{13} \mathrm{C} \mathrm{NMR}$ $\left(\mathrm{CDCl}_{3}\right) \delta 157.4,152.7,151.3,149.7,142.7,136.4,132.9,129.2,127.3,126.1,124.9,123.9$ 119.8, 116.9, 43.2 ppm; IR (KBr) v 3067, 1628, 1570, 1286, 1122, 871, $772 \mathrm{~cm}^{-1}$; MS (ESI) $\mathrm{m} / \mathrm{z} 322.8\left([\mathrm{M}+\mathrm{Na}]^{+}\right)$; HRMS (ESI) calcd for $\mathrm{C}_{15} \mathrm{H}_{12} \mathrm{~N}_{2} \mathrm{O}_{3} \mathrm{~S}\left([\mathrm{M}+\mathrm{Na}]^{+}\right), 323.0461$; found, 323.0450 .

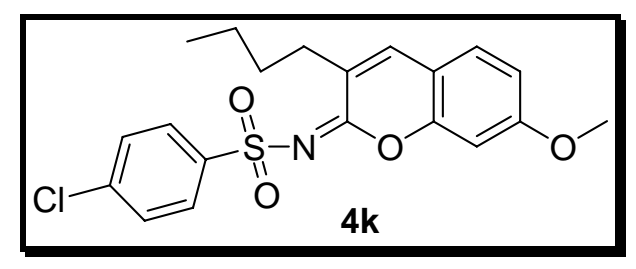

Pale yellow crystal; mp. $187-190{ }^{\circ} \mathrm{C} ;{ }^{1} \mathrm{H}$ NMR $\left(\mathrm{CDCl}_{3}\right) \delta 8.02\left(\mathrm{dd}, J_{1}=2.0 \mathrm{~Hz}, J_{2}=8.0 \mathrm{~Hz}\right.$, 2H), $7.47(\mathrm{~m}, 3 \mathrm{H}), 7.35(\mathrm{~m}, 1 \mathrm{H}), 6.89(\mathrm{~m}, 2 \mathrm{H}), 3.87(\mathrm{~s}, 3 \mathrm{H}), 2.47$ (t, J= $7.8 \mathrm{~Hz}, 2 \mathrm{H}), 1.56(\mathrm{~m}$ 2H), $1.34(\mathrm{~m}, 2 \mathrm{H}), 0.90(\mathrm{~m}, 3 \mathrm{H}) \mathrm{ppm} ;{ }^{13} \mathrm{C} \mathrm{NMR}\left(\mathrm{CDCl}_{3}\right) \delta 162.6,159.5,153.6,141.3,138.72$, $138.68,129.0,128.8,128.6,127.4,114.6,113.5,100.3,56.2,30.3,30.0,22.5,14.0$ ppm; IR $(\mathrm{KBr})$ v 3092, 2958, 1635, 1539, 1312, 1158, 1086, $837 \mathrm{~cm}^{-1}$; MS (ESI) m/z $427.8\left([\mathrm{M}+\mathrm{Na}]^{+}\right)$; HRMS (ESI) calcd for $\mathrm{C}_{20} \mathrm{H}_{20} \mathrm{ClNO}_{4} \mathrm{~S}\left([\mathrm{M}+\mathrm{Na}]^{+}\right)$, 428.0694; found, 428.0676. 


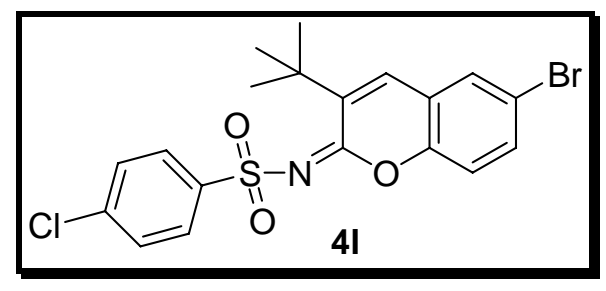

White crystal; mp. $195-198{ }^{\circ} \mathrm{C} ;{ }^{1} \mathrm{H}$ NMR $\left(\mathrm{CDCl}_{3}\right) \delta 8.00(\mathrm{~d}, J=8.8 \mathrm{~Hz}, 2 \mathrm{H}), 7.61(\mathrm{~m}, 2 \mathrm{H})$, $7.51(\mathrm{~m}, 3 \mathrm{H}), 7.32(\mathrm{~d}, J=8.4 \mathrm{~Hz}, 1 \mathrm{H}), 1.36(\mathrm{~s}, 9 \mathrm{H}) \mathrm{ppm} ;{ }^{13} \mathrm{C} \mathrm{NMR}\left(\mathrm{CDCl}_{3}\right) \delta 156.6,150.7$ $141.2,138.99,138.95,135.8,134.4,130.5,129.3,128.6,121.3,118.4,118.0,35.7,28.9$ ppm; IR $(\mathrm{KBr}) \vee$ 3080, 2959, 1619, 1542, 1314, 1157, 1081, $833 \mathrm{~cm}^{-1} ; \mathrm{MS}(\mathrm{ESI}) \mathrm{m} / \mathrm{z} 476.1$ $\left([\mathrm{M}+\mathrm{Na}]^{+}\right)$; HRMS (ESI) calcd for $\mathrm{C}_{19} \mathrm{H}_{17} \mathrm{BrClNO}_{3} \mathrm{~S}\left([\mathrm{M}+\mathrm{Na}]^{+}\right), 475.9693$; found, 475.9693 .

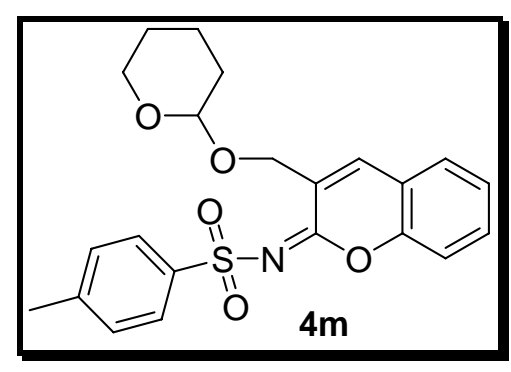

White crystal; mp. $158-161{ }^{\circ} \mathrm{C} ;{ }^{1} \mathrm{H} \operatorname{NMR}\left(\mathrm{CDCl}_{3}\right) \delta 7.99(\mathrm{~d}, J=8.4 \mathrm{~Hz}, 2 \mathrm{H}), 7.84(\mathrm{~s}, 1 \mathrm{H})$, $7.52(\mathrm{~m}, 2 \mathrm{H}), 7.42(\mathrm{~d}, J=8.4 \mathrm{~Hz}, 1 \mathrm{H}), 7.31(\mathrm{~m}, 3 \mathrm{H}), 4.69(\mathrm{~m}, 2 \mathrm{H}), 4.40\left(\mathrm{dd}, J_{1}=1.2 \mathrm{~Hz}, J_{2}=\right.$ $16.0 \mathrm{~Hz}, 1 \mathrm{H}), 3.85(\mathrm{~m}, 1 \mathrm{H}), 3.53(\mathrm{~m}, 1 \mathrm{H}), 2.41(\mathrm{~s}, 3 \mathrm{H}), 1.89-1.56(\mathrm{~m}, 6 \mathrm{H}) \mathrm{ppm} ;{ }^{13} \mathrm{C} \mathrm{NMR}$ $\left(\mathrm{CDCl}_{3}\right) \delta 156.5,152.2,143.5,139.2,137.0,131.7,129.5,128.3,127.9,127.7,125.9,119.6$ 116.6, 99.3, 63.9, 62.9, 30.7, 25.5, 21.8, 19.8 ppm; IR (KBr) v 3062, 2945, 1635, 1558, 1325, 1163, 1029, $831 \mathrm{~cm}^{-1}$; MS (ESI) m/z $436.2\left([\mathrm{M}+\mathrm{Na}]^{+}\right)$; HRMS (ESI) calcd for $\mathrm{C}_{22} \mathrm{H}_{23} \mathrm{NO}_{5} \mathrm{~S}$ $\left([\mathrm{M}+\mathrm{Na}]^{+}\right), 436.1189 ;$ found, 436.1171. 


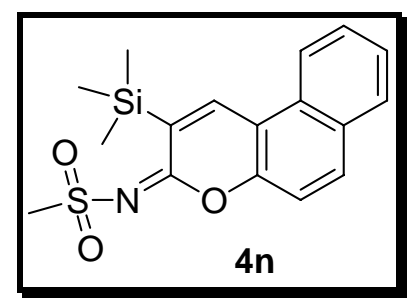

Yellow solid. Mp. $139-143{ }^{\circ} \mathrm{C} ;{ }^{1} \mathrm{H}$ NMR $\left(\mathrm{CDCl}_{3}\right) \delta 8.55(\mathrm{~s}, 1 \mathrm{H}), 8.28(\mathrm{~d}, J=8.4 \mathrm{~Hz}, 1 \mathrm{H})$, $7.99(\mathrm{~d}, J=9.2 \mathrm{~Hz}, 1 \mathrm{H}), 7.91(\mathrm{~d}, J=8.0 \mathrm{~Hz}, 1 \mathrm{H}), 7.71(\mathrm{~m}, 1 \mathrm{H}), 7.59(\mathrm{~m}, 2 \mathrm{H}), 3.17(\mathrm{~s}, 3 \mathrm{H})$, $0.42(\mathrm{~s}, 9 \mathrm{H}) \mathrm{ppm} ;{ }^{13} \mathrm{C} \mathrm{NMR}\left(\mathrm{CDCl}_{3}\right) \delta 160.7,153.2,144.2,133.8,130.9,129.6,129.3,128.7$ 128.6, 126.7, 121.4, 117.0, 114.0, 43.2, -1.57 ppm; IR (KBr) v 3049, 2933, 1675, 1635, 1512, 1315, 1241, 1144, 966, $816 \mathrm{~cm}^{-1}$; MS (ESI) m/z $367.8\left([\mathrm{M}+\mathrm{Na}]^{+}\right)$; HRMS (ESI) calcd for $\mathrm{C}_{17} \mathrm{H}_{19} \mathrm{NO}_{3} \mathrm{SSi}\left([\mathrm{M}+\mathrm{Na}]^{+}\right), 368.0747$; found, 368.0744.

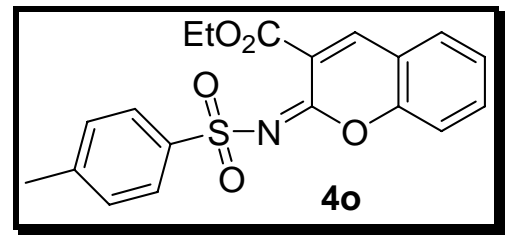

Yellow solid; mp. $151-153{ }^{\circ} \mathrm{C} ;{ }^{1} \mathrm{H}$ NMR $\left(\mathrm{CDCl}_{3}\right) \delta 8.29(\mathrm{~s}, 1 \mathrm{H}), 7.99(\mathrm{~d}, J=8.8 \mathrm{~Hz}, 2 \mathrm{H})$, $7.65(\mathrm{~m}, 1 \mathrm{H}), 7.58(\mathrm{~d}, J=7.6 \mathrm{~Hz}, 1 \mathrm{H}), 7.46(\mathrm{~m}, 2 \mathrm{H}), 7.30(\mathrm{~m}, 2 \mathrm{H}), 4.33(\mathrm{q}, J=7.2 \mathrm{~Hz}, 2 \mathrm{H})$, $2.41(\mathrm{~s}, 3 \mathrm{H}), 1.30(\mathrm{t}, J=7.4 \mathrm{~Hz}, 3 \mathrm{H}) \mathrm{ppm} ;{ }^{13} \mathrm{C} \mathrm{NMR}\left(\mathrm{CDCl}_{3}\right) \delta 162.8,154.1,153.5,145.7$ $143.3,139.5,134.6,129.7,129.4,127.3,126.2,121.2,118.2,117.0,62.4,21.7,14.2$ ppm; IR $(\mathrm{KBr})$ v 3077, 1700, 1630, 1584, 1309, 1239, 1090, $833 \mathrm{~cm}^{-1}$; MS (ESI) m/z $394.2\left([\mathrm{M}+\mathrm{Na}]^{+}\right)$; HRMS (ESI) calcd for $\mathrm{C}_{19} \mathrm{H}_{17} \mathrm{NO}_{5} \mathrm{~S}\left([\mathrm{M}+\mathrm{Na}]^{+}\right), 394.0720$; found, 394.0704.

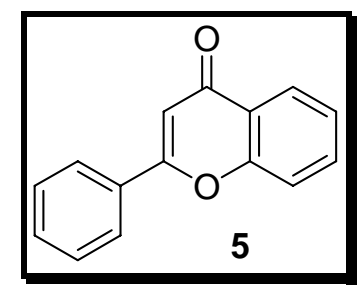

White solid; mp. 139-141 ${ }^{\circ} \mathrm{C} ;{ }^{1} \mathrm{H}$ NMR $\left(\mathrm{CDCl}_{3}\right) \delta 7.77(\mathrm{~s}, 1 \mathrm{H}), 7.68\left(\mathrm{dd}, J_{1}=1.6 \mathrm{~Hz}, J_{2}=8.4\right.$ 
$\mathrm{Hz}, 2 \mathrm{H}), 7.50(\mathrm{~m}, 2 \mathrm{H}), 7.41(\mathrm{~m}, 3 \mathrm{H}), 7.32$ (d, $J=8.4 \mathrm{~Hz}, 1 \mathrm{H}), 7.27(\mathrm{~m}, 1 \mathrm{H}) \mathrm{ppm} ;{ }^{13} \mathrm{C}$ NMR $\left(\mathrm{CDCl}_{3}\right) \delta 160.7,163.6,140.0,134.8,131.5,129.0,128.7,128.6,128.4,128.1,124.7,119.8$ 116.5 ppm; IR $(\mathrm{KBr}) \vee 3056,1719,1612,1453,1123,1118,961,751 \mathrm{~cm}^{-1} ; \mathrm{MS}(\mathrm{ESI}) \mathrm{m} / \mathrm{z}$ $244.8\left([\mathrm{M}+\mathrm{Na}]^{+}\right) ;$HRMS (ESI) calcd for $\mathrm{C}_{15} \mathrm{H}_{10} \mathrm{O}_{2}\left([\mathrm{M}+\mathrm{Na}]^{+}\right), 245.0573$; found, 245.0567.

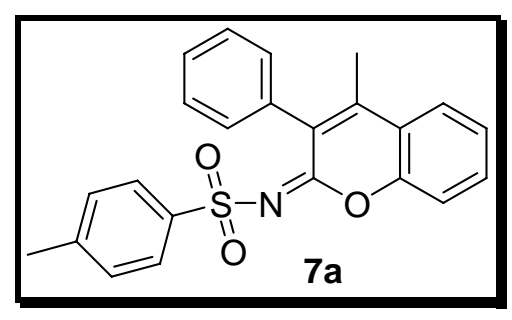

Yellow crystal; mp. $236-240{ }^{\circ} \mathrm{C} ;{ }^{1} \mathrm{H}$ NMR $\left(\mathrm{CDCl}_{3}\right) \delta 7.82(\mathrm{~d}, J=8.4 \mathrm{~Hz}, 2 \mathrm{H}), 7.70\left(\mathrm{dd}, J_{1}=\right.$ 1.6 Hz, $\left.J_{2}=8.0 \mathrm{~Hz}, 1 \mathrm{H}\right), 7.57(\mathrm{~m}, 1 \mathrm{H}), 7.48(\mathrm{~m}, 1 \mathrm{H}), 7.40(\mathrm{~m}, 4 \mathrm{H}), 7.23(\mathrm{~m}, 4 \mathrm{H}), 2.37(\mathrm{~s}$, 3H), $2.28(\mathrm{~s}, 3 \mathrm{H}) \mathrm{ppm} ;{ }^{13} \mathrm{C} \mathrm{NMR}\left(\mathrm{CDCl}_{3}\right) \delta 158.4,151.8,147.6,142.8,140.0,134.2,132.0$, $130.2,129.2,128.8,128.5,128.4,127.2,125.7,125.4,120.9,117.1,21.7,17.0$ ppm; IR (KBr) $v \quad 3067,3044,1620,1539,1299,1155,1081,782 \mathrm{~cm}^{-1} ; \mathrm{MS}(\mathrm{ESI}) \mathrm{m} / \mathrm{z} 412.2\left([\mathrm{M}+\mathrm{Na}]^{+}\right)$ HRMS (ESI) calcd for $\mathrm{C}_{23} \mathrm{H}_{19} \mathrm{NO}_{3} \mathrm{~S}\left([\mathrm{M}+\mathrm{Na}]^{+}\right), 412.0978$; found, 412.0964 .

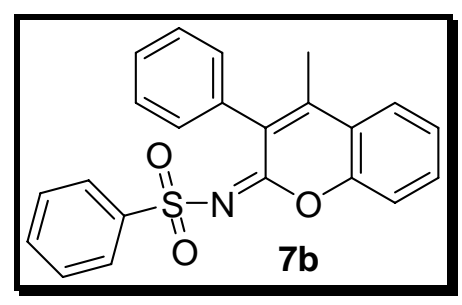

Brown solid; mp. 215-219 ${ }^{\circ} \mathrm{C} ;{ }^{1} \mathrm{H}$ NMR $\left(\mathrm{CDCl}_{3}\right) \delta 7.93(\mathrm{~d}, J=7.2 \mathrm{~Hz}, 2 \mathrm{H}), 7.69$ (d, $J=8.4$ $\mathrm{Hz}, 1 \mathrm{H}), 7.57(\mathrm{~m}, 1 \mathrm{H}), 7.41(\mathrm{~m}, 8 \mathrm{H}), 7.24(\mathrm{~d}, J=6.8 \mathrm{~Hz}, 2 \mathrm{H}), 2.27(\mathrm{~s}, 3 \mathrm{H}) \mathrm{ppm} ;{ }^{13} \mathrm{C} \mathrm{NMR}$ $\left(\mathrm{CDCl}_{3}\right) \delta 158.6,151.6,148.0,142.8,134.1,132.2,132.0,130.2,128.61,128.58,128.5$ 128.4, 127.0, 125.8, 120.8, 116.9, 17.0 ppm; IR (KBr) v 3060, 1618, 1539, 1317, 1303, 1151, 1080, 838, 783, $756 \mathrm{~cm}^{-1}$; MS (ESI) m/z $398.2\left([\mathrm{M}+\mathrm{Na}]^{+}\right)$; HRMS (ESI) calcd for 
$\mathrm{C}_{22} \mathrm{H}_{17} \mathrm{NO}_{3} \mathrm{~S}\left([\mathrm{M}+\mathrm{Na}]^{+}\right), 398.0821$; found, 398.0803.

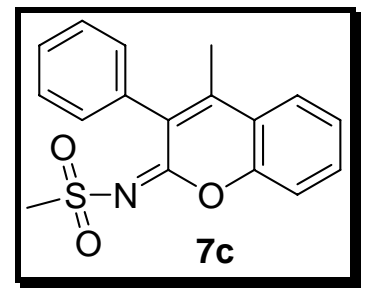

White crystal; mp. $233-236{ }^{\circ} \mathrm{C} ;{ }^{1} \mathrm{H} \operatorname{NMR}\left(\mathrm{CDCl}_{3}\right) \delta 7.71(\mathrm{~d}, J=7.6 \mathrm{~Hz}, 1 \mathrm{H}), 7.58(\mathrm{~m}$, 2H), $7.41(\mathrm{~m}, 4 \mathrm{H}), 7.25(\mathrm{~d}, J=7.6 \mathrm{~Hz}, 2 \mathrm{H}), 2.95(\mathrm{~s}, 3 \mathrm{H}), 2.28(\mathrm{~s}, 3 \mathrm{H}) \mathrm{ppm} ;{ }^{13} \mathrm{C} \mathrm{NMR}\left(\mathrm{CDCl}_{3}\right)$ $\delta 158.6,151.7,147.6,134.3,131.9,130.2,128.5,128.4,128.3,125.7,125.3,120.8,117.4$, 43.1, 16.9 ppm; IR (KBr) v 3021, 1623, 1558, 1301, 1137, 974, 850, $795 \mathrm{~cm}^{-1}$; MS (ESI) m/z $335.8\left([\mathrm{M}+\mathrm{Na}]^{+}\right)$; HRMS (ESI) calcd for $\mathrm{C}_{17} \mathrm{H}_{15} \mathrm{NO}_{3} \mathrm{~S}\left([\mathrm{M}+\mathrm{Na}]^{+}\right), 336.0665$; found, 336.0650.

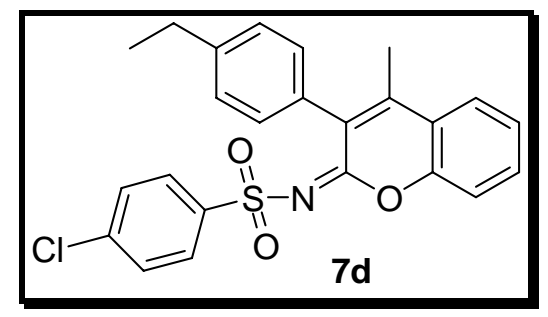

White solid; mp. $240-243{ }^{\circ} \mathrm{C} ;{ }^{1} \mathrm{H}$ NMR $\left(\mathrm{CDCl}_{3}\right) \delta 7.86(\mathrm{~d}, J=8.8 \mathrm{~Hz}, 2 \mathrm{H}), 7.72\left(\mathrm{dd}, J_{1}=0.8\right.$ $\left.\mathrm{Hz}, J_{2}=8.0 \mathrm{~Hz}, 1 \mathrm{H}\right), 7.59(\mathrm{~m}, 1 \mathrm{H}), 7.48(\mathrm{~m}, 1 \mathrm{H}), 7.38(\mathrm{~m}, 3 \mathrm{H}), 7.26(\mathrm{~d}, J=8.0 \mathrm{~Hz}, 2 \mathrm{H}), 7.15$ $(\mathrm{d}, J=8.0 \mathrm{~Hz}, 2 \mathrm{H}), 2.71(\mathrm{q}, J=7.6 \mathrm{~Hz}, 2 \mathrm{H}), 2.31(\mathrm{~s}, 3 \mathrm{H}), 1.29(\mathrm{t}, J=7.6 \mathrm{~Hz}, 3 \mathrm{H}) \mathrm{ppm} ;{ }^{13} \mathrm{C}$ $\operatorname{NMR}\left(\mathrm{CDCl}_{3}\right) \delta 159.1,151.6,148.3,144.6,141.5,138.4,132.1,131.2,130.1,128.9,128.7$, 128.6, 128.0, 125.9, 125.5, 121.0, 117.0, 28.9, 17.1, 15.6 ppm; IR (KBr) v 3088, 2965, 1620, 1539, 1325, 1156, 1080, $830 \mathrm{~cm}^{-1}$; MS (ESI) m/z $460.2\left([\mathrm{M}+\mathrm{Na}]^{+}\right)$; HRMS (ESI) calcd for $\mathrm{C}_{24} \mathrm{H}_{20} \mathrm{ClNO}_{3} \mathrm{~S}\left([\mathrm{M}+\mathrm{Na}]^{+}\right), 460.0745$; found, 460.0725 . 


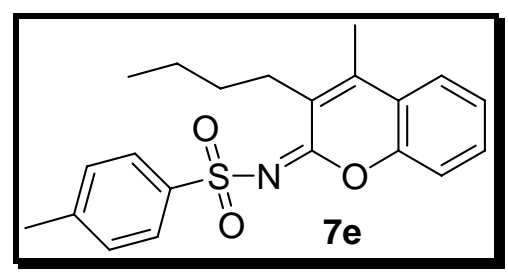

Brown crystal; mp. $197-199{ }^{\circ} \mathrm{C} ;{ }^{1} \mathrm{H}$ NMR $\left(\mathrm{CDCl}_{3}\right) \delta 7.97(\mathrm{~d}, J=8.8 \mathrm{~Hz}, 2 \mathrm{H}), 7.60\left(\mathrm{dd}, J_{1}=\right.$ 1.2 Hz, $\left.J_{2}=7.6 \mathrm{~Hz}, 1 \mathrm{H}\right), 7.45(\mathrm{~m}, 1 \mathrm{H}), 7.30(\mathrm{~m}, 4 \mathrm{H}), 2.65(\mathrm{t}, J=7.6 \mathrm{~Hz}, 2 \mathrm{H}), 2.40(\mathrm{~s}, 3 \mathrm{H})$, $2.37(\mathrm{~s}, 3 \mathrm{H}), 1.47(\mathrm{~m}, 2 \mathrm{H}), 1.36(\mathrm{~m}, 2 \mathrm{H}), 0.89(\mathrm{t}, J=7.6 \mathrm{~Hz}, 3 \mathrm{H}) \mathrm{ppm} ;{ }^{13} \mathrm{C} \mathrm{NMR}\left(\mathrm{CDCl}_{3}\right) \delta$ $158.6,151.1,146.1,143.0,139.9,131.1,129.3,128.0,127.4,125.5,124.7,120.9,116.7,30.5$, 27.5, 22.9, 21.7, 15.2, 14.1 ppm; IR (KBr) v 2950, 2870, 1620, 1558, 1312, 1154, 1082, 769 $\mathrm{cm}^{-1}$; MS (ESI) $\mathrm{m} / \mathrm{z} 391.8\left([\mathrm{M}+\mathrm{Na}]^{+}\right)$; HRMS (ESI) calcd for $\mathrm{C}_{21} \mathrm{H}_{23} \mathrm{NO}_{3} \mathrm{~S}\left([\mathrm{M}+\mathrm{Na}]^{+}\right)$, 392.1291; found, 392.1307.

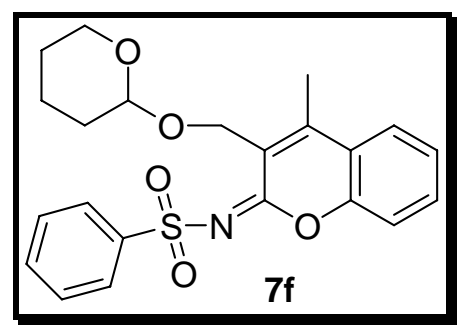

Yellow crystal; mp. $172-174{ }^{\circ} \mathrm{C} ;{ }^{1} \mathrm{H} \operatorname{NMR}\left(\mathrm{CDCl}_{3}\right) \delta 8.10\left(\mathrm{dd}, J_{1}=1.2 \mathrm{~Hz}, J_{2}=7.6 \mathrm{~Hz}, 2 \mathrm{H}\right)$, $7.69\left(\mathrm{dd}, J_{1}=1.6 \mathrm{~Hz}, J_{2}=8.0 \mathrm{~Hz}, 1 \mathrm{H}\right), 7.52(\mathrm{~m}, 4 \mathrm{H}), 7.37(\mathrm{~m}, 2 \mathrm{H}), 4.82(\mathrm{~d}, J=11.6 \mathrm{~Hz}, 1 \mathrm{H})$, $4.71(\mathrm{~m}, 1 \mathrm{H}), 4.56(\mathrm{~d}, J=10.8 \mathrm{~Hz}, 1 \mathrm{H}), 3.86(\mathrm{~m}, 1 \mathrm{H}), 3.43(\mathrm{~m}, 1 \mathrm{H}), 2.56(\mathrm{~s}, 3 \mathrm{H}), 1.60(\mathrm{~m}$, 2H), $1.49(\mathrm{~m}, 4 \mathrm{H}) \mathrm{ppm} ;{ }^{13} \mathrm{C} \mathrm{NMR}\left(\mathrm{CDCl}_{3}\right) \delta 158.7,151.8,151.0,142.7,132.4,132.3,128.8$ $127.4,125.8,125.3,123.6,120.8,117.0,99.7,62.7,61.7,30.7,25.5,19.7,15.5$ ppm; IR (KBr) $v$ 2935, 2884, 1621, 1539, 1449, 1317, 1163, 1086, 862, $762 \mathrm{~cm}^{-1} ; \mathrm{MS}(\mathrm{ESI}) \mathrm{m} / \mathrm{z} 436.2$ $\left([\mathrm{M}+\mathrm{Na}]^{+}\right)$; HRMS (ESI) calcd for $\mathrm{C}_{22} \mathrm{H}_{23} \mathrm{NO}_{5} \mathrm{~S}\left([\mathrm{M}+\mathrm{Na}]^{+}\right), 436.1189$; found, 436.1169 .

\section{References and Notes}

1. (a) Danheiser, R. L.; Miller, R. F.; Brisbois, R. G.; Park, S. Z. J. Org. Chem.; 1990; 55, 1959-1964. (b) 
Hazen, G. G.; Bollinger, F. W.; Roberts, F. E.; Russ, W. K.; Seman, J. J.; Staskiewicz, S. Org. Synth. 1998,Coll. Vol. 9, 400; 1996, Vol. 73, 144.

2. Caution: Although the mixture of sulfonyl azides is the safest of a group of diazo compounds, one should keep in mind the inherent instability, shock sensitivity, and explosive power of azides. All users should exercise appropriate caution. 
Copies of ${ }^{1} \mathrm{H}$ and ${ }^{13} \mathrm{C}$ NMR Spectra for All Products
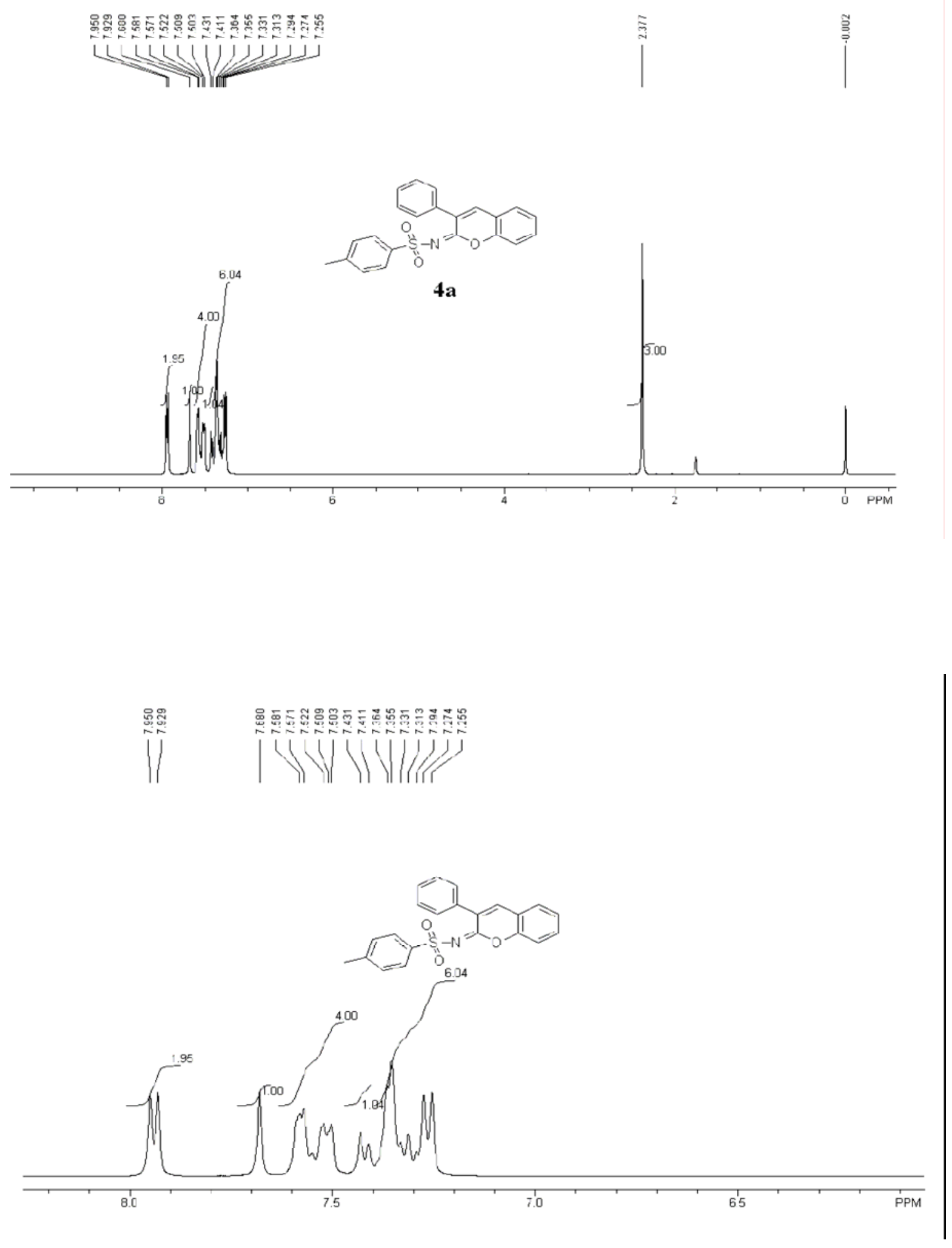

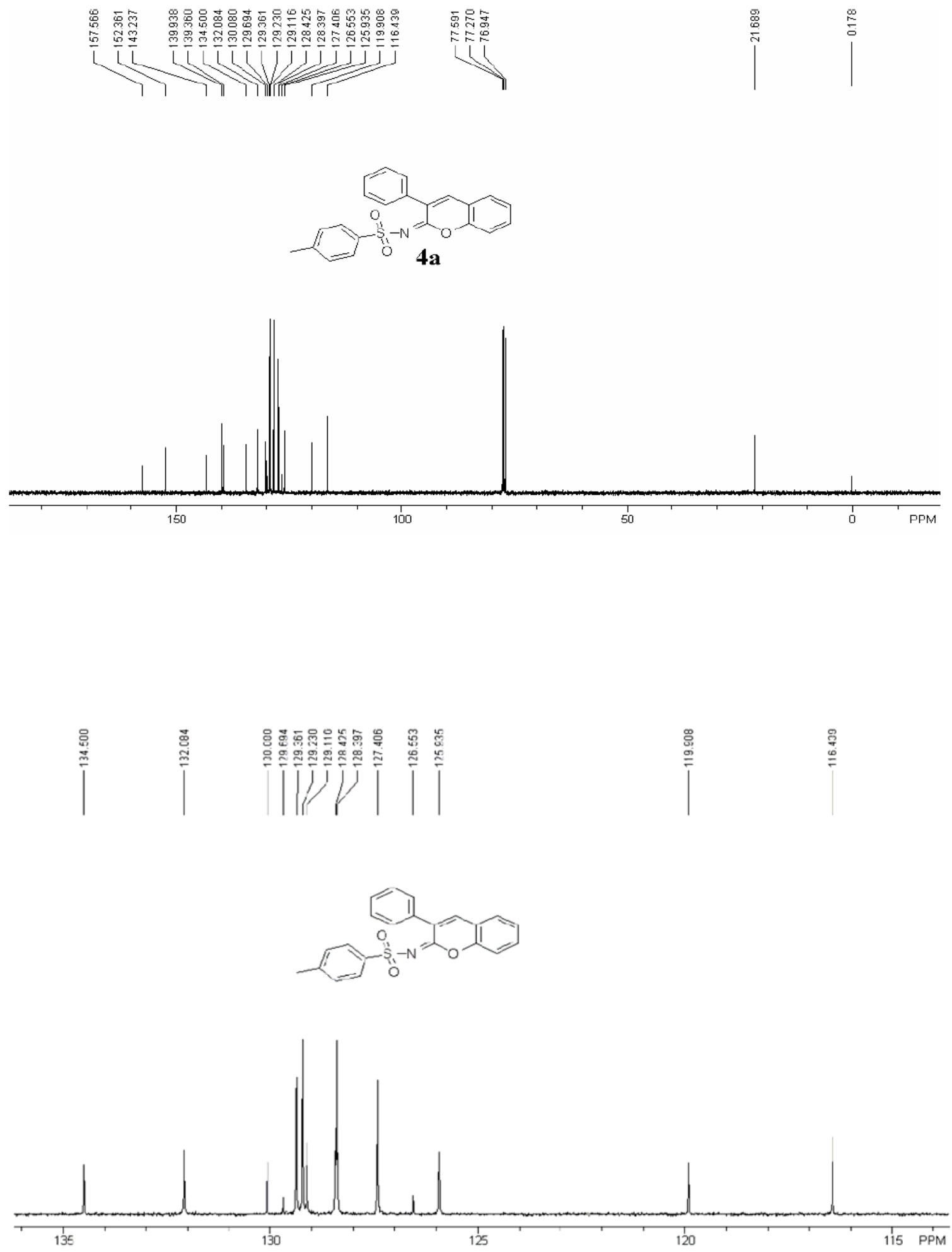


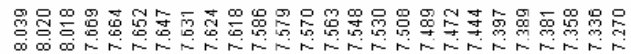

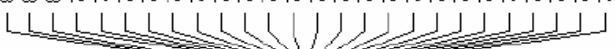

7 iा
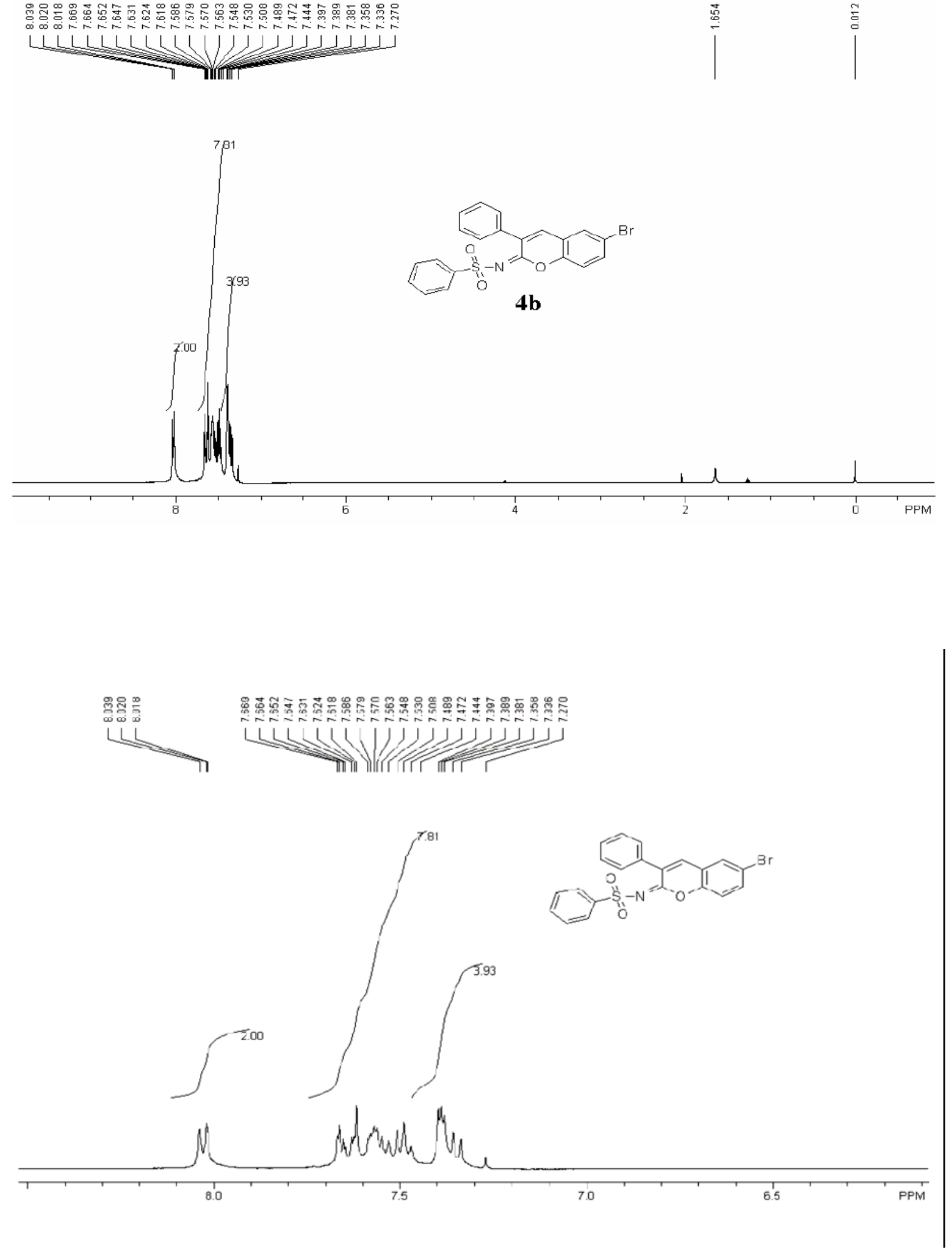

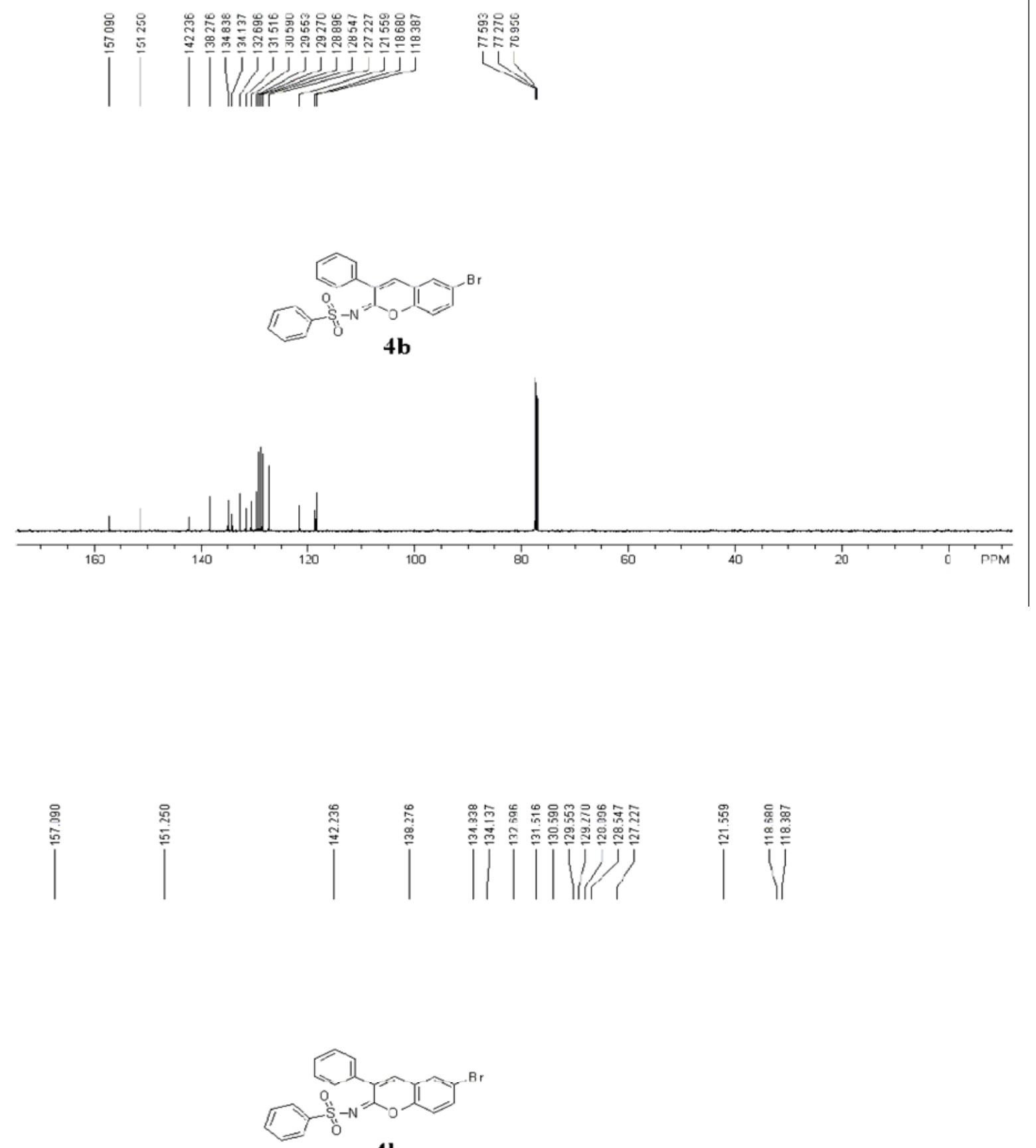

4b

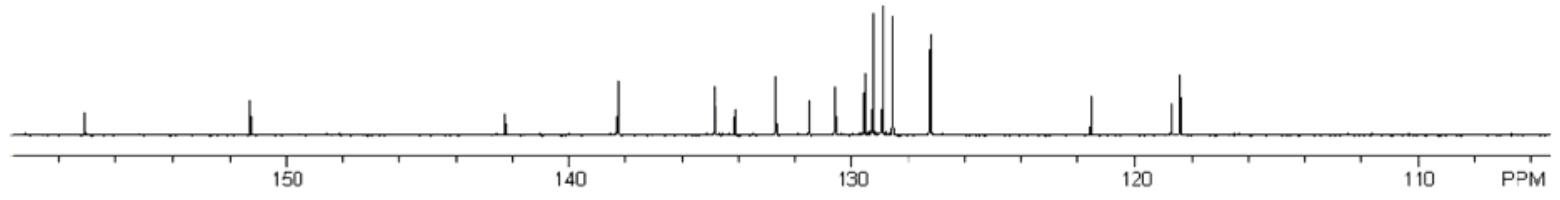




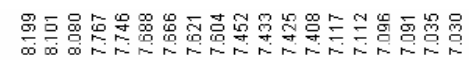

Liviuj
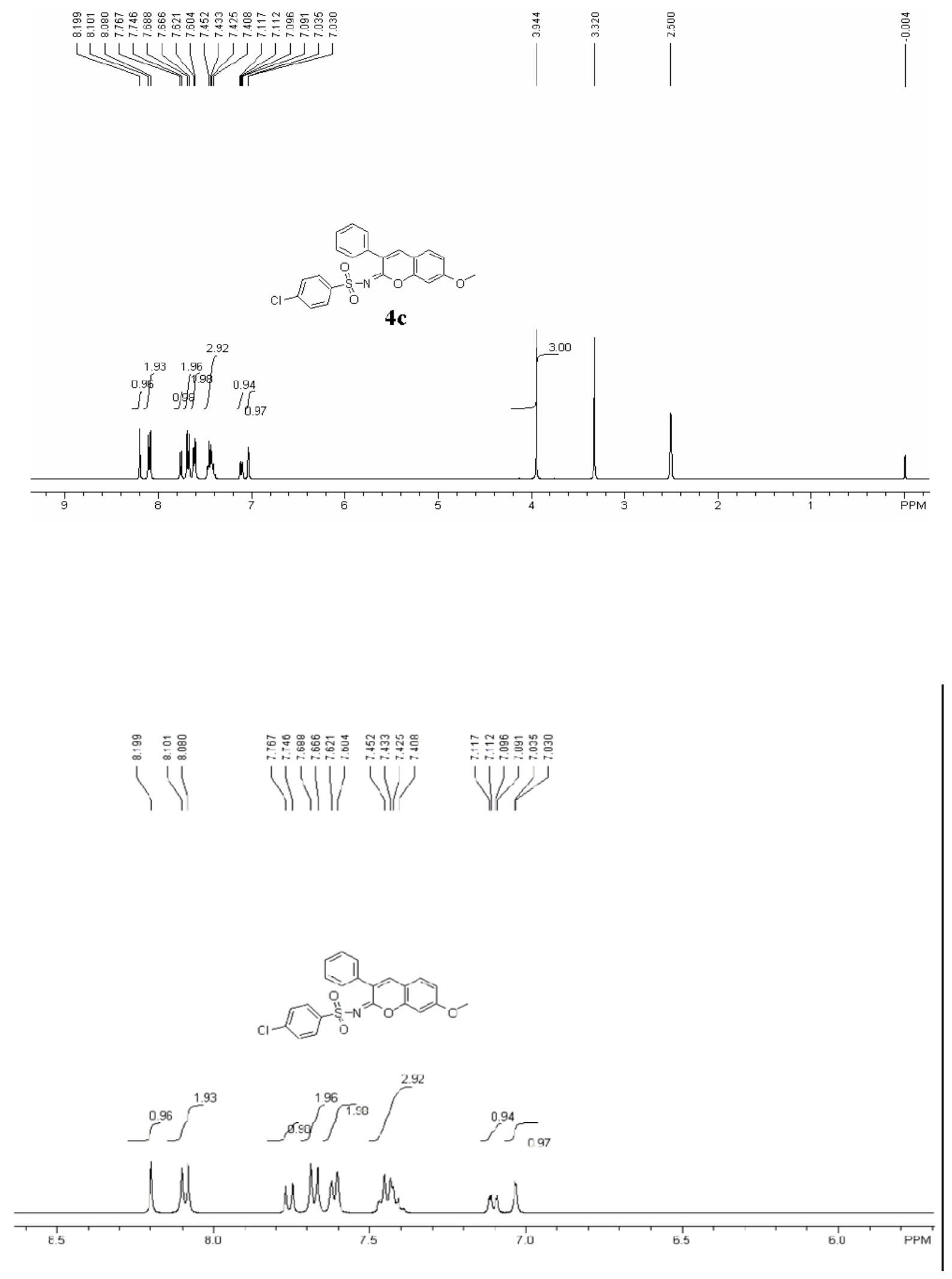


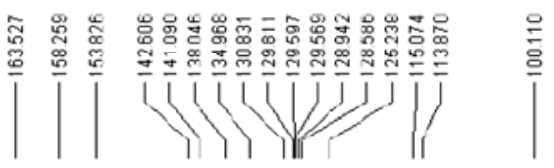
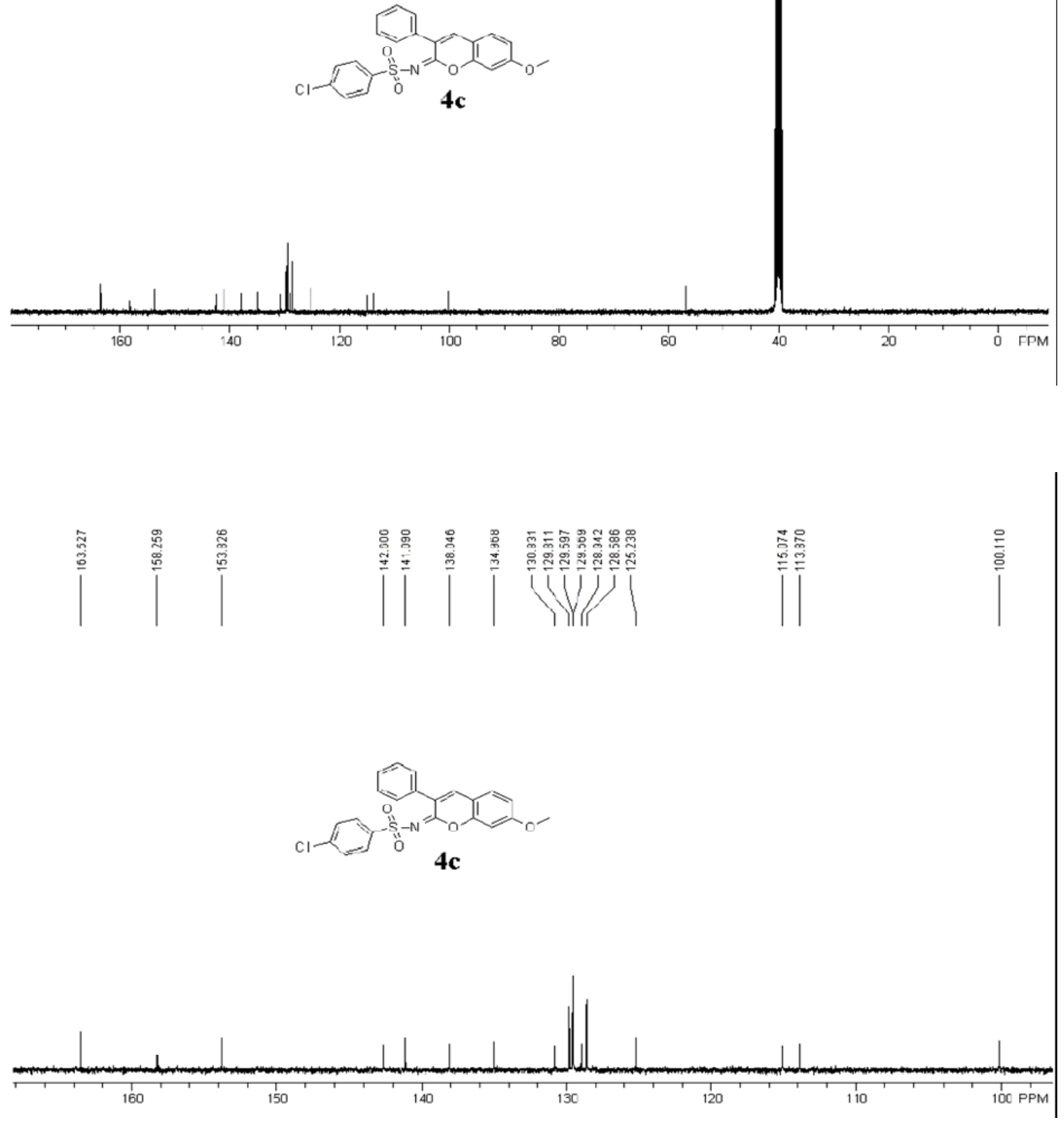


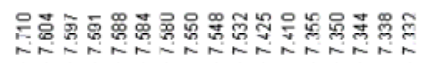

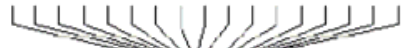

7i i

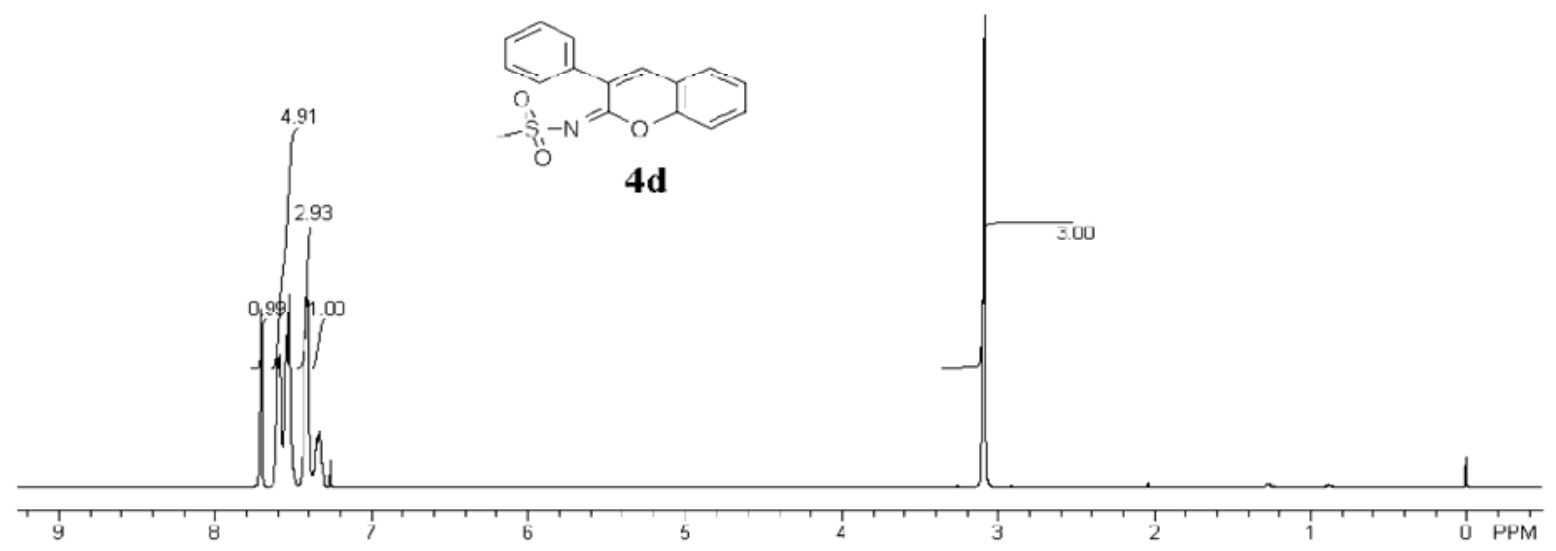

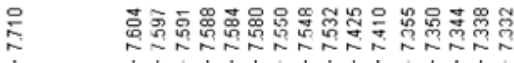

(†
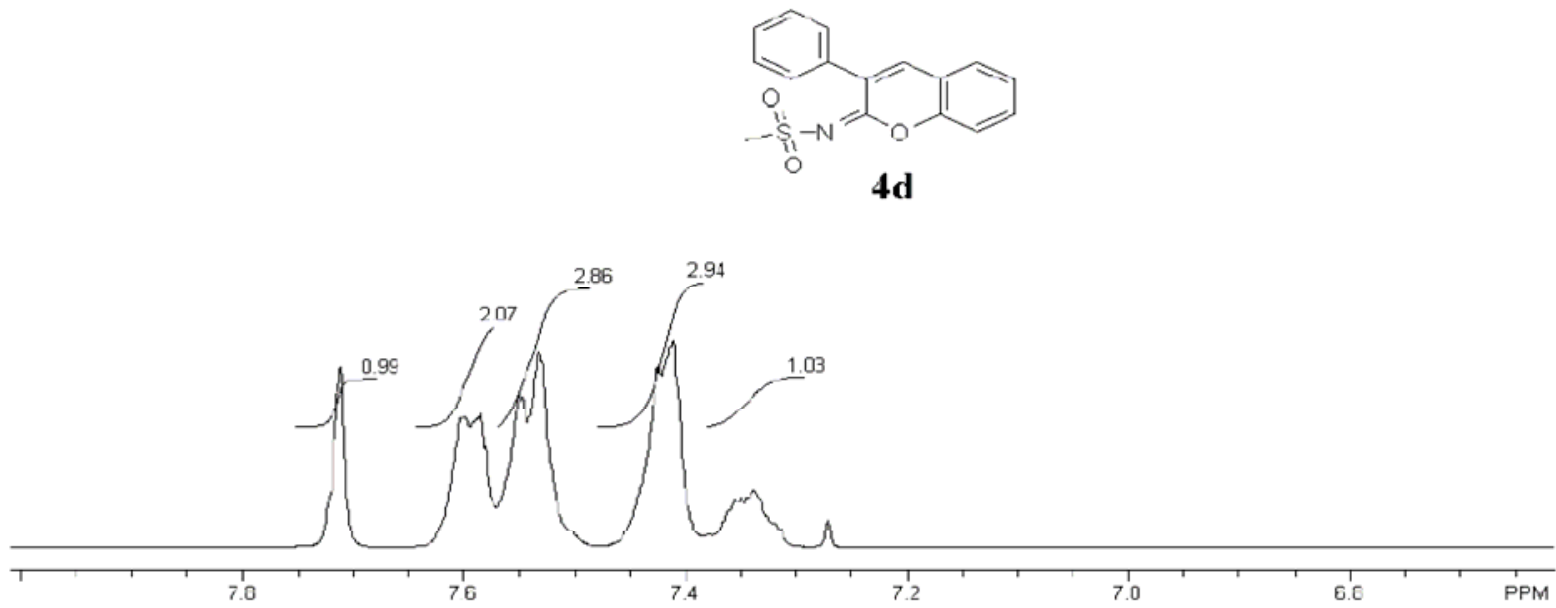

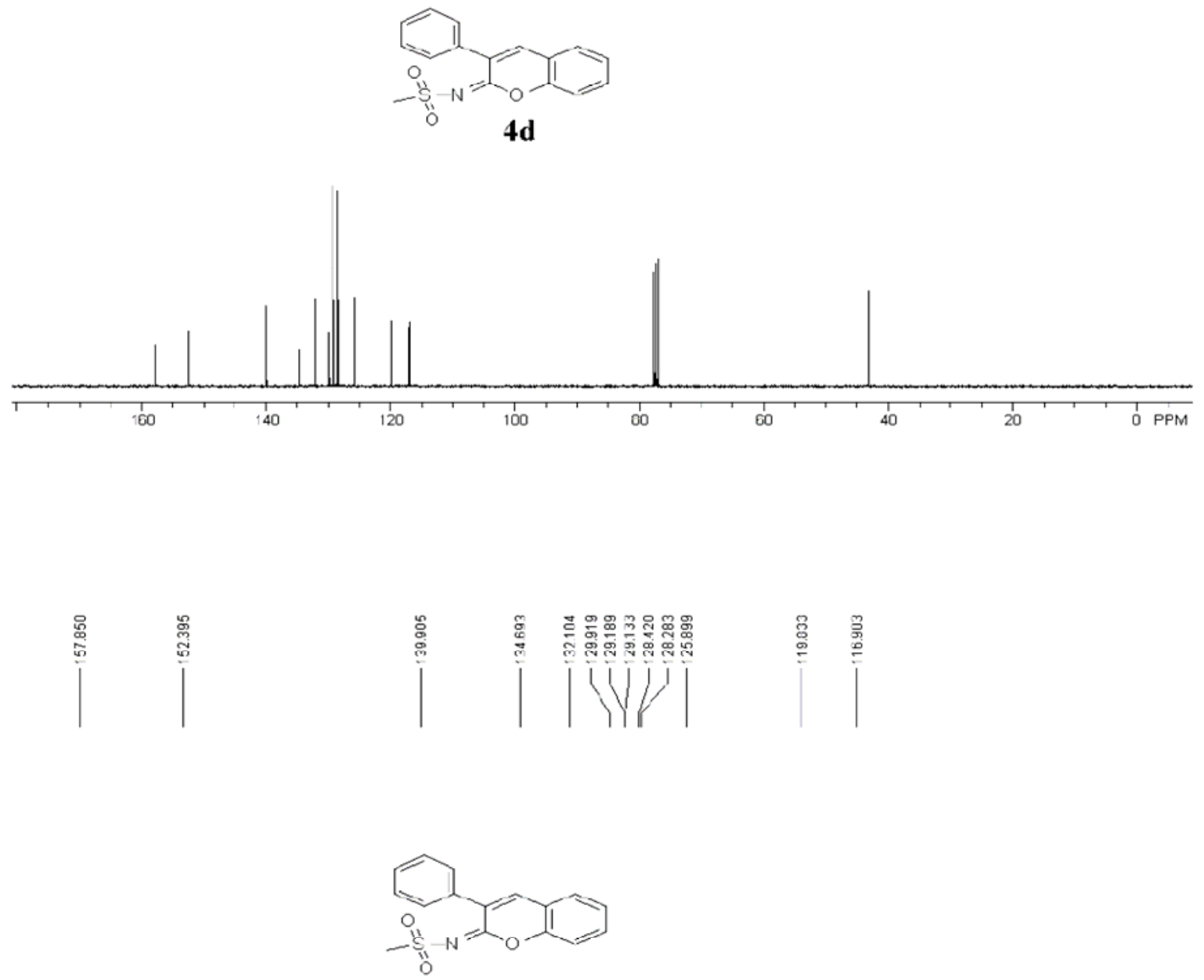

4d

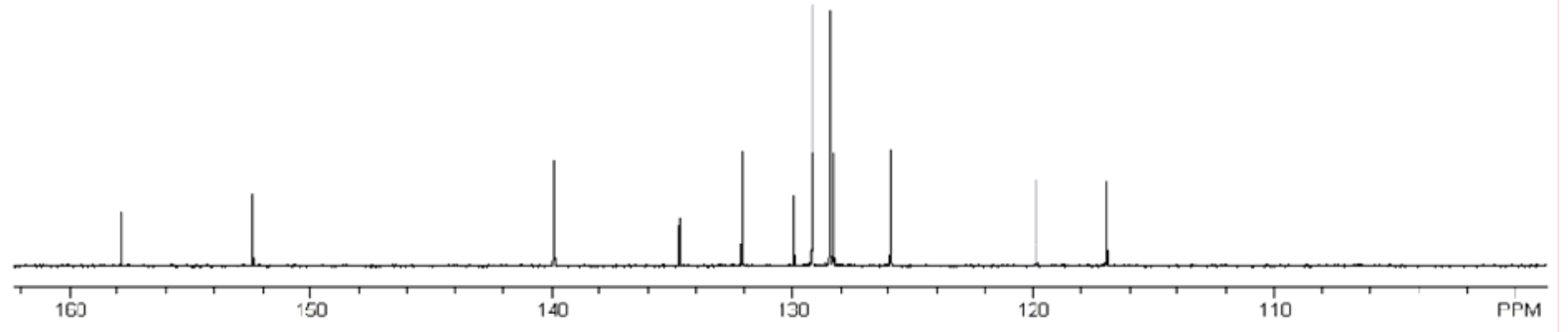




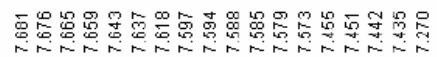

LiiLiMUJjJjJjJ

ili
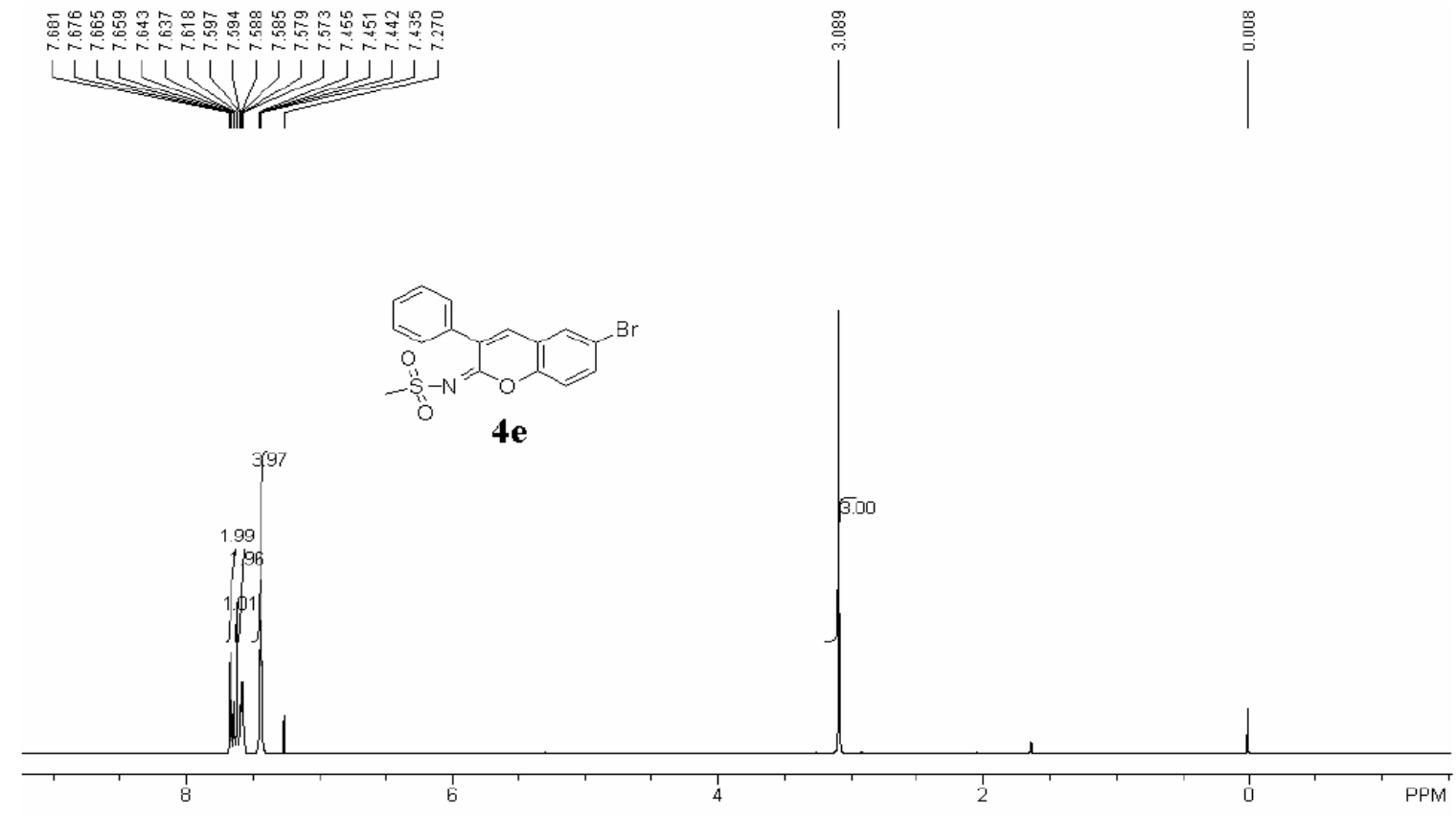

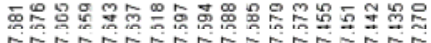

닌
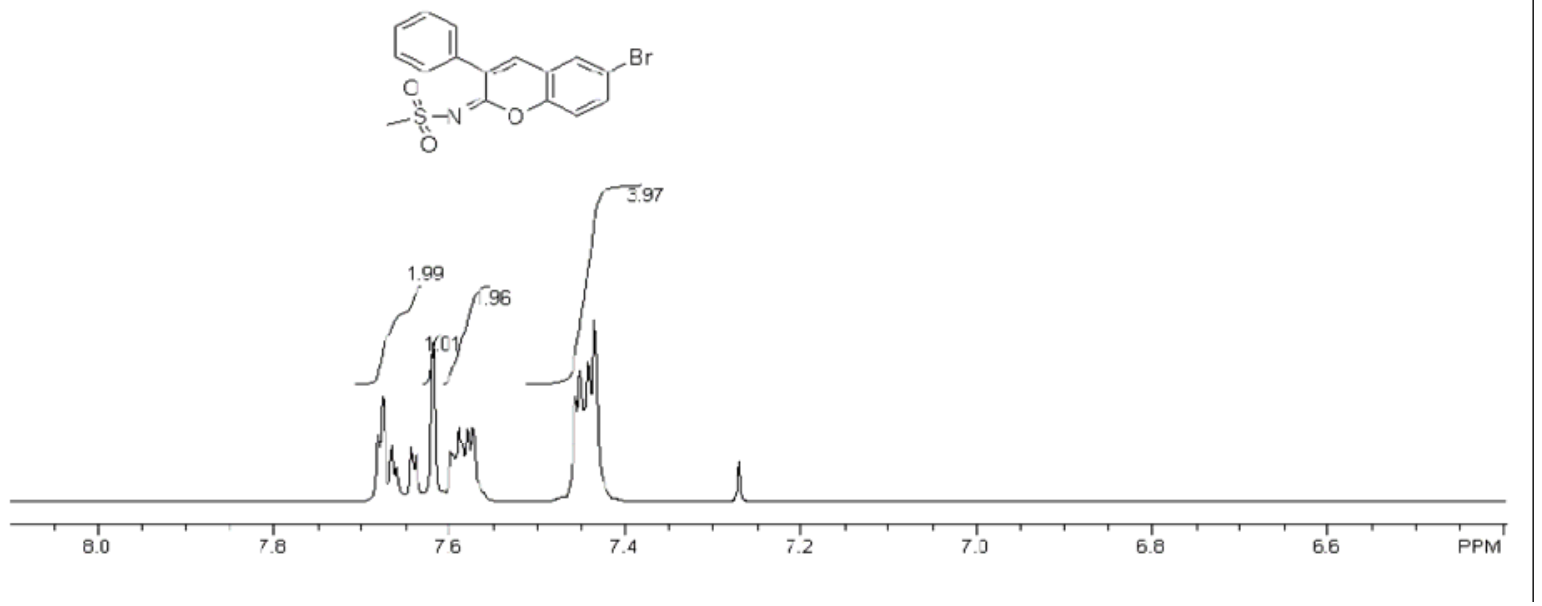

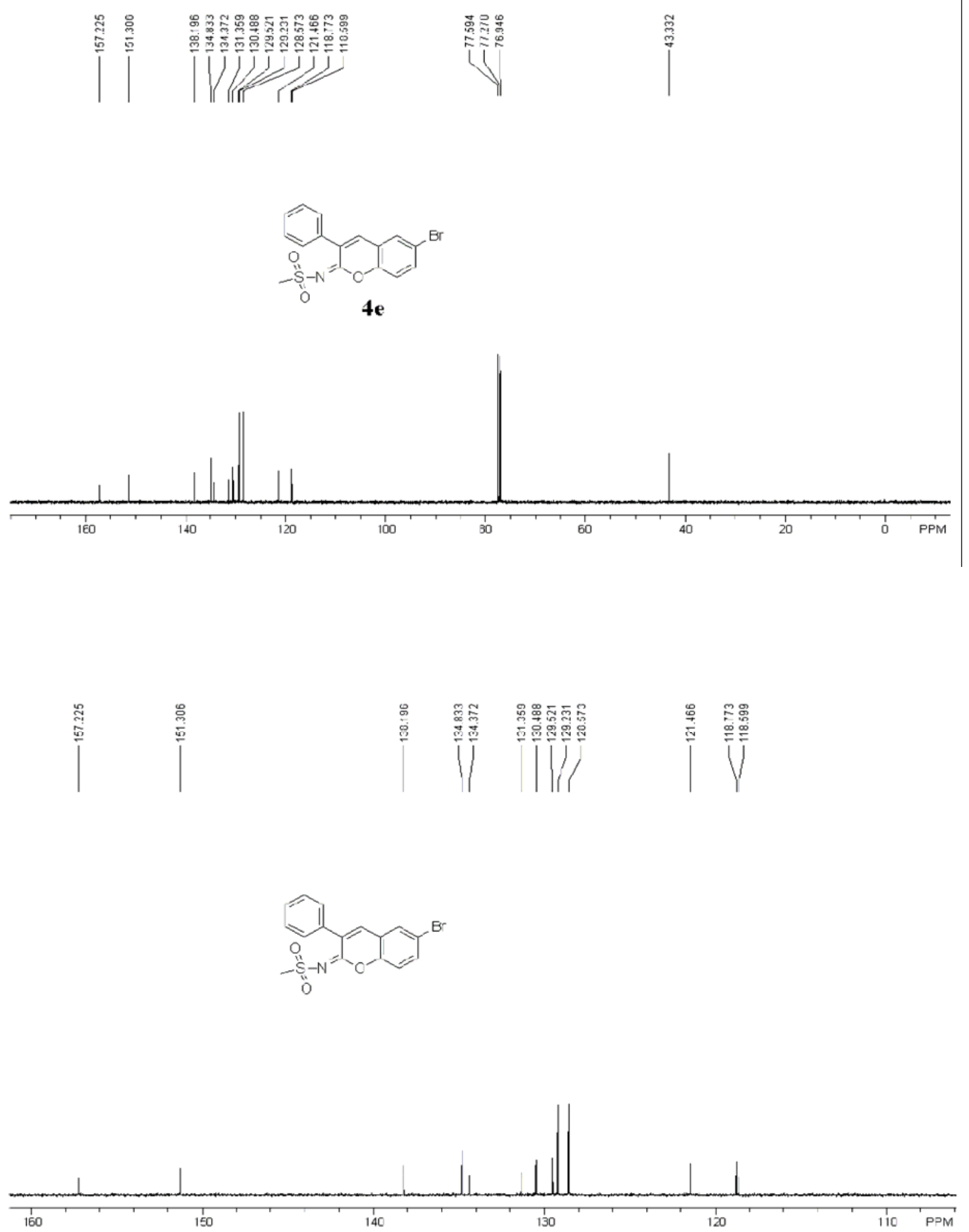


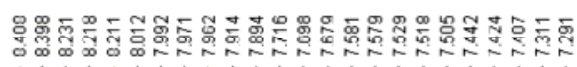

$4+44 / 1 / 11111$

त 7 in
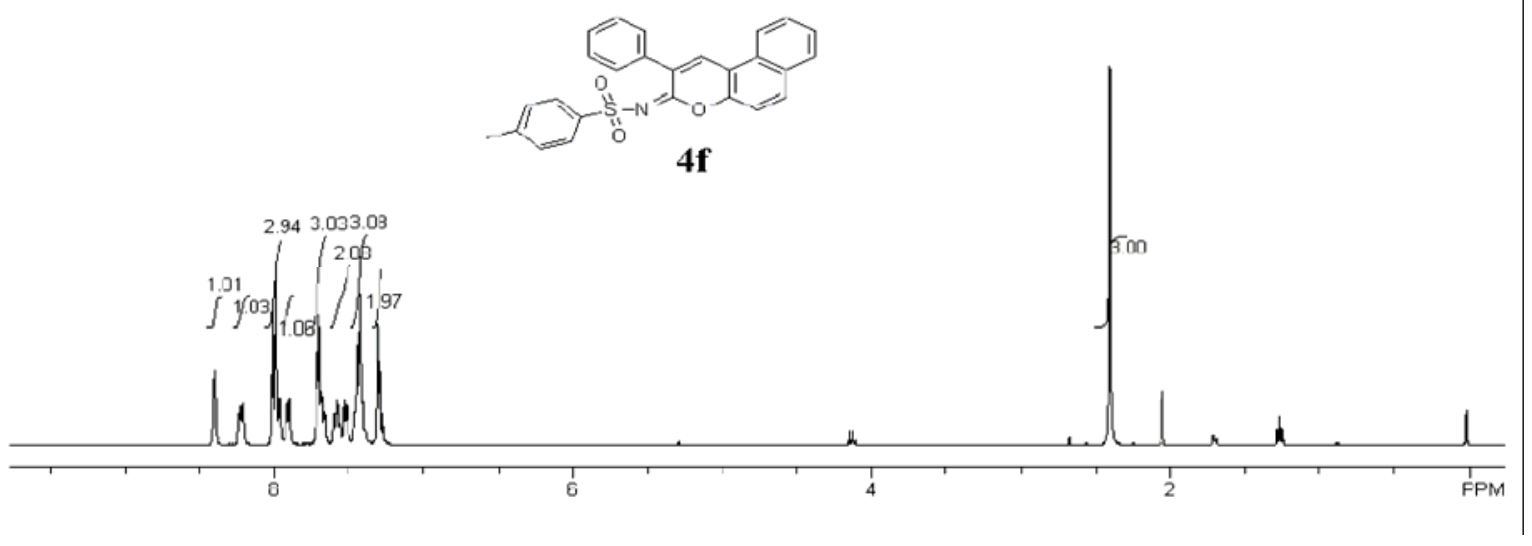

U
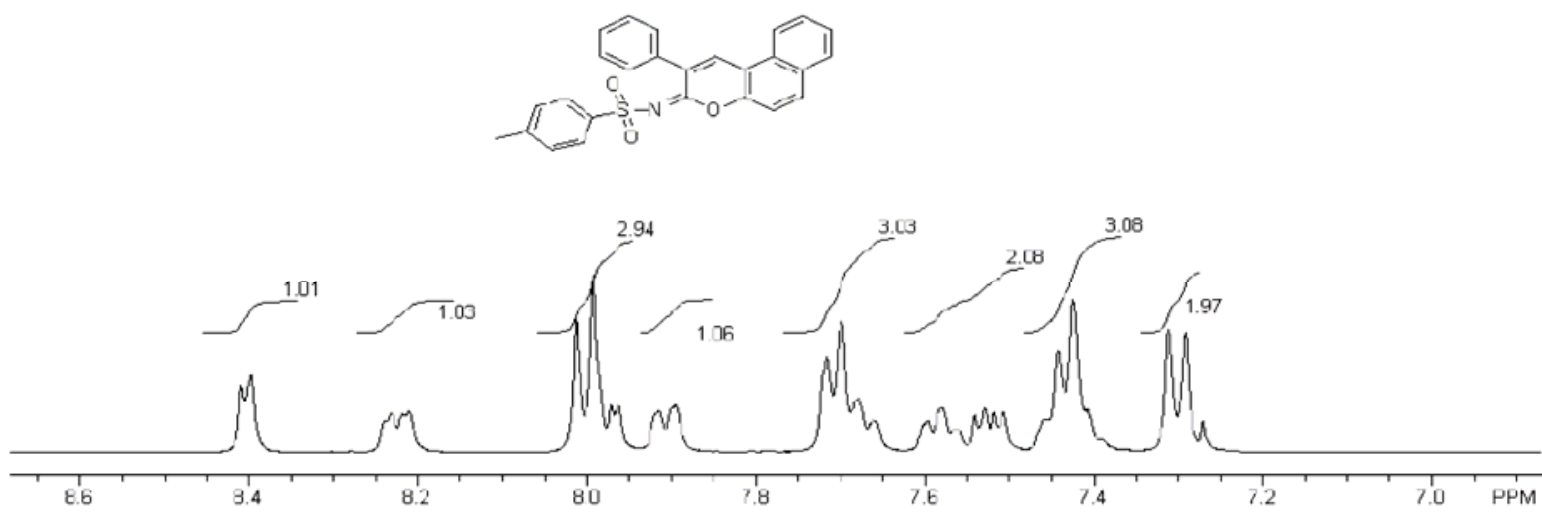

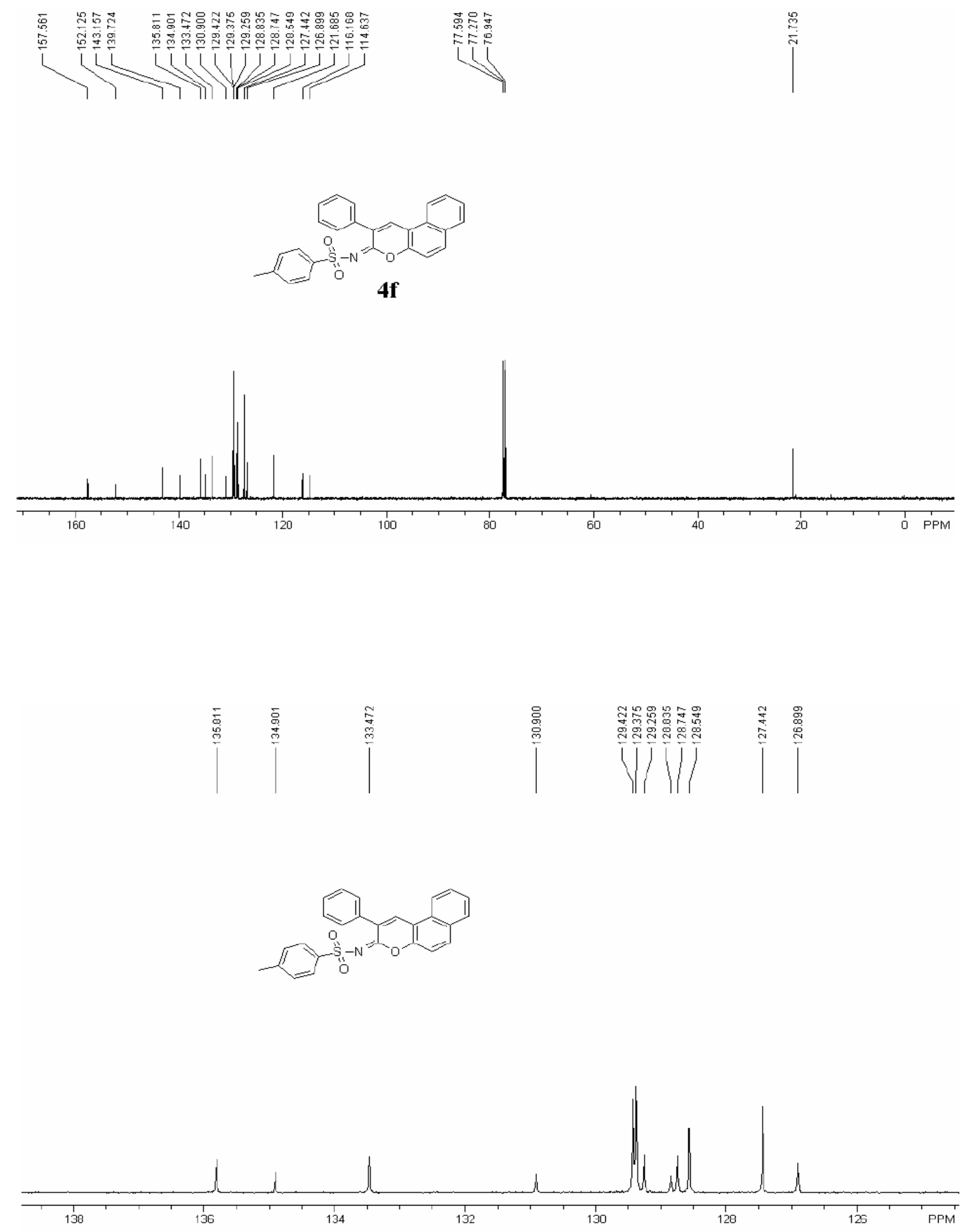


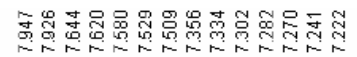

UMUVIDIJ

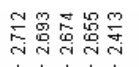

Uy

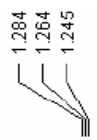

İ
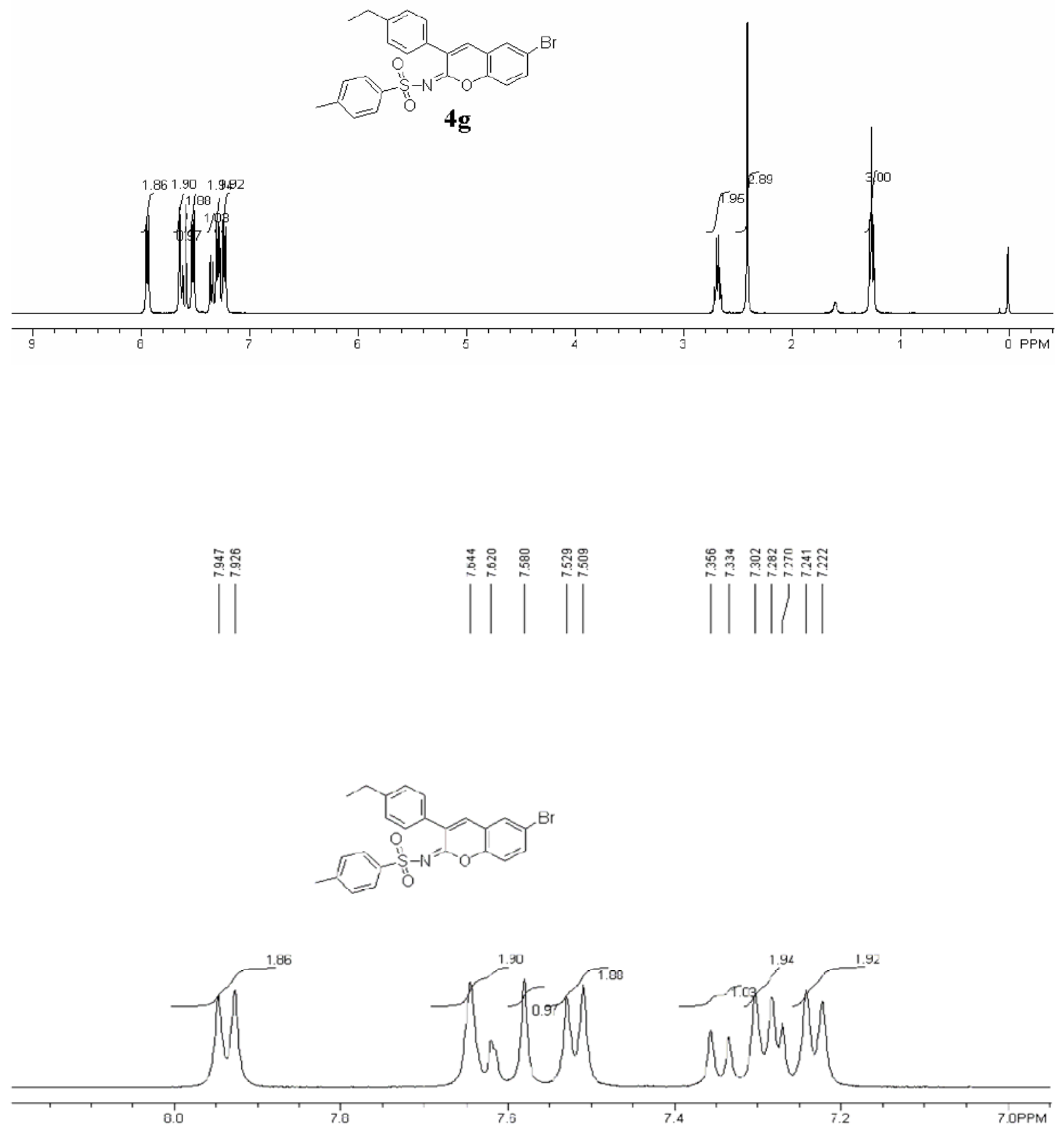

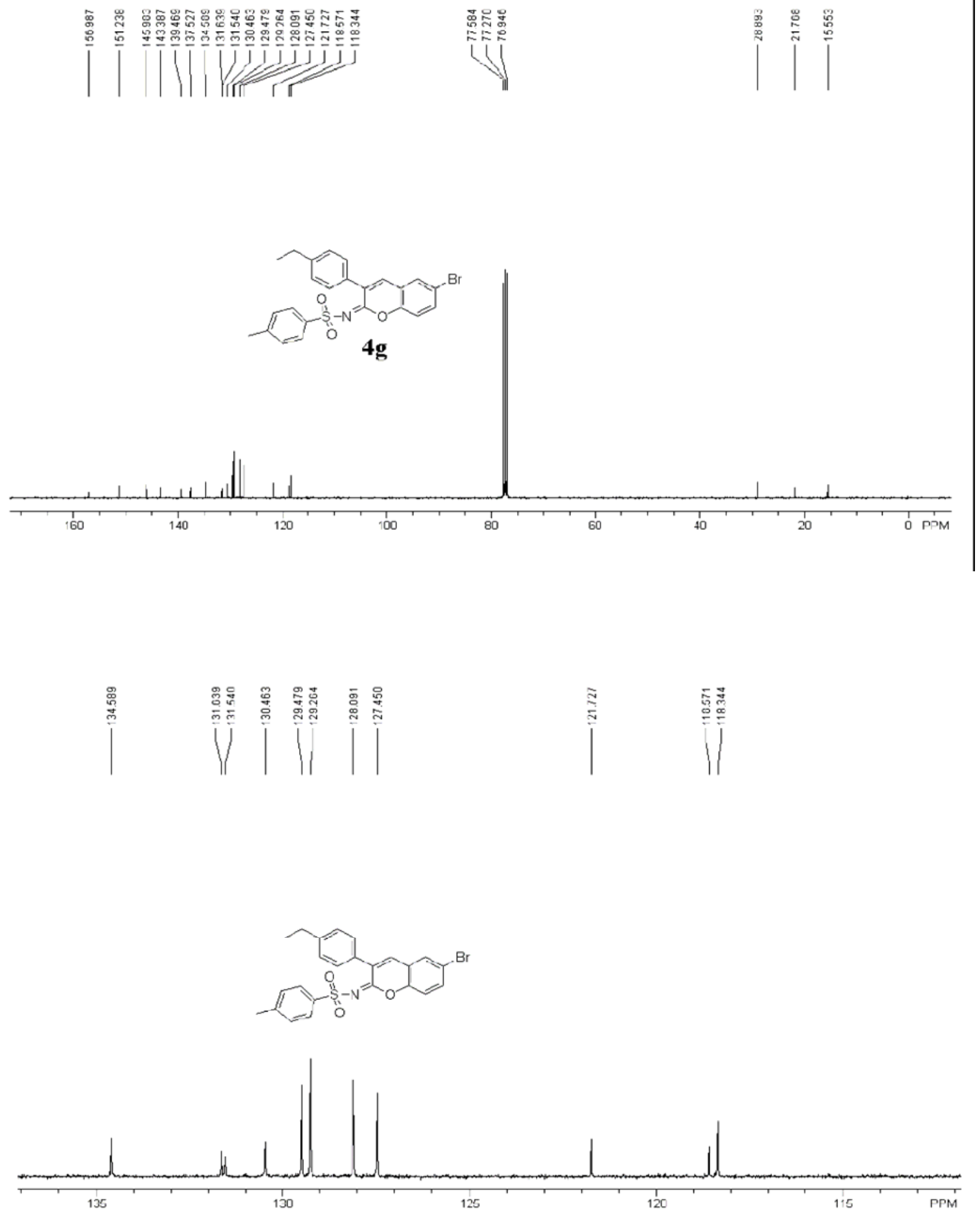


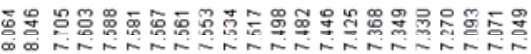

(

7าiा
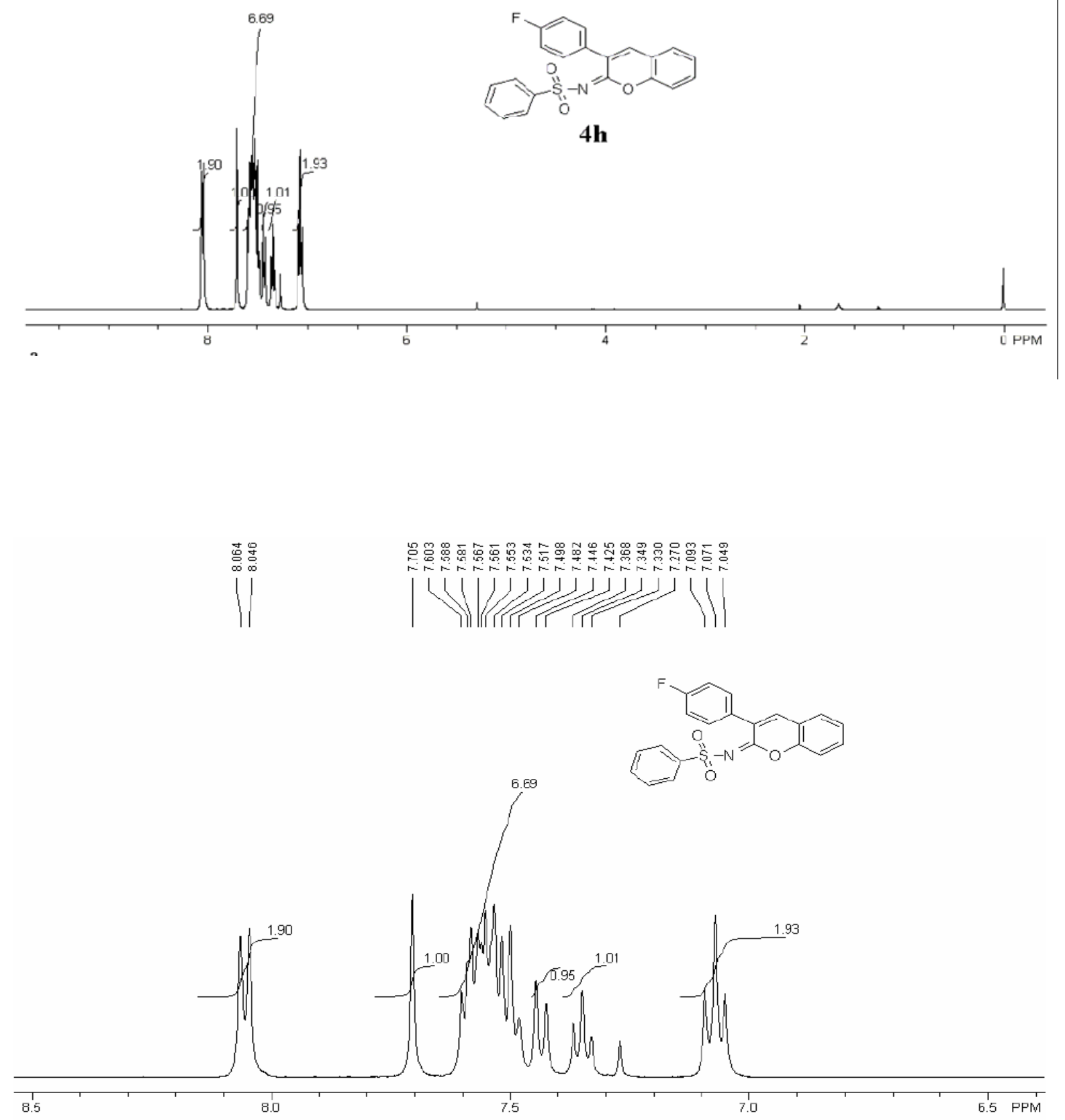


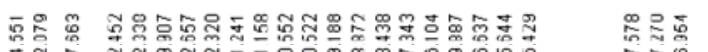

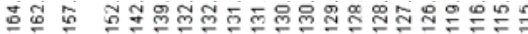

$1+1$
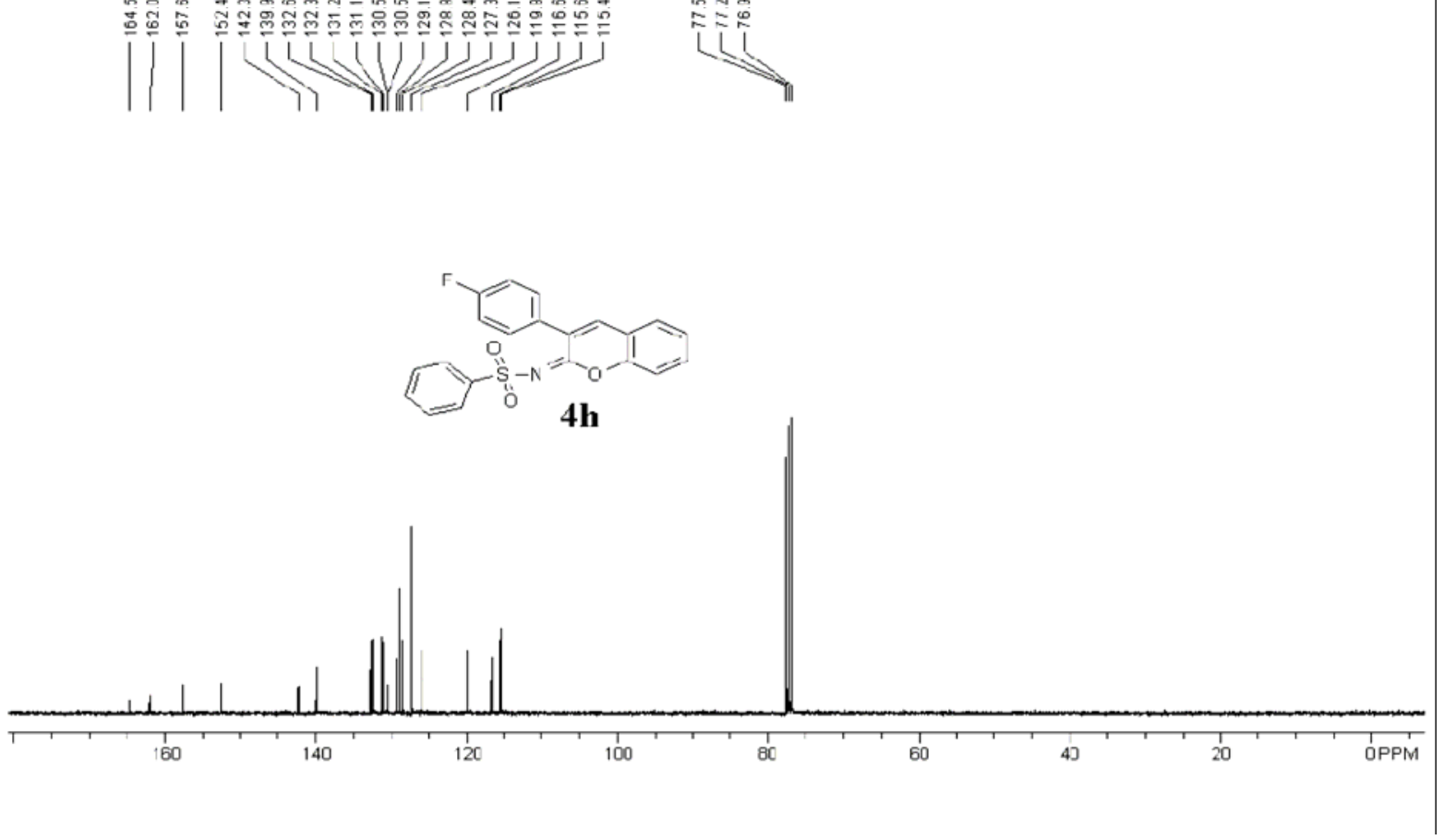

|
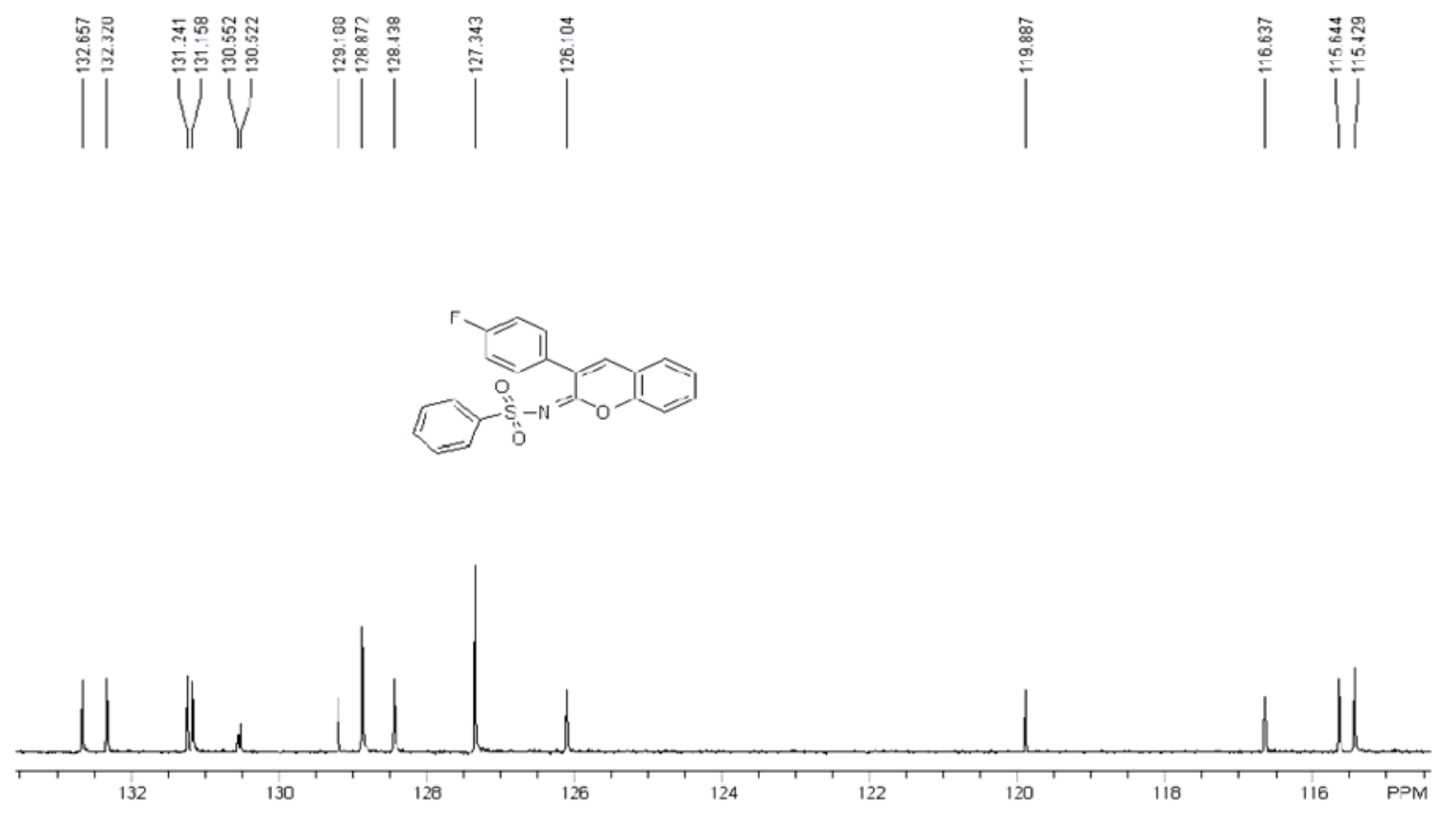


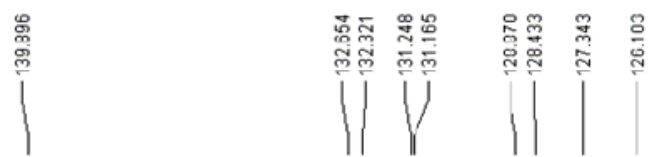

ธ్

||$^{m}||^{\infty}$

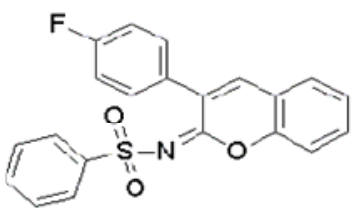

4h-DEPT135
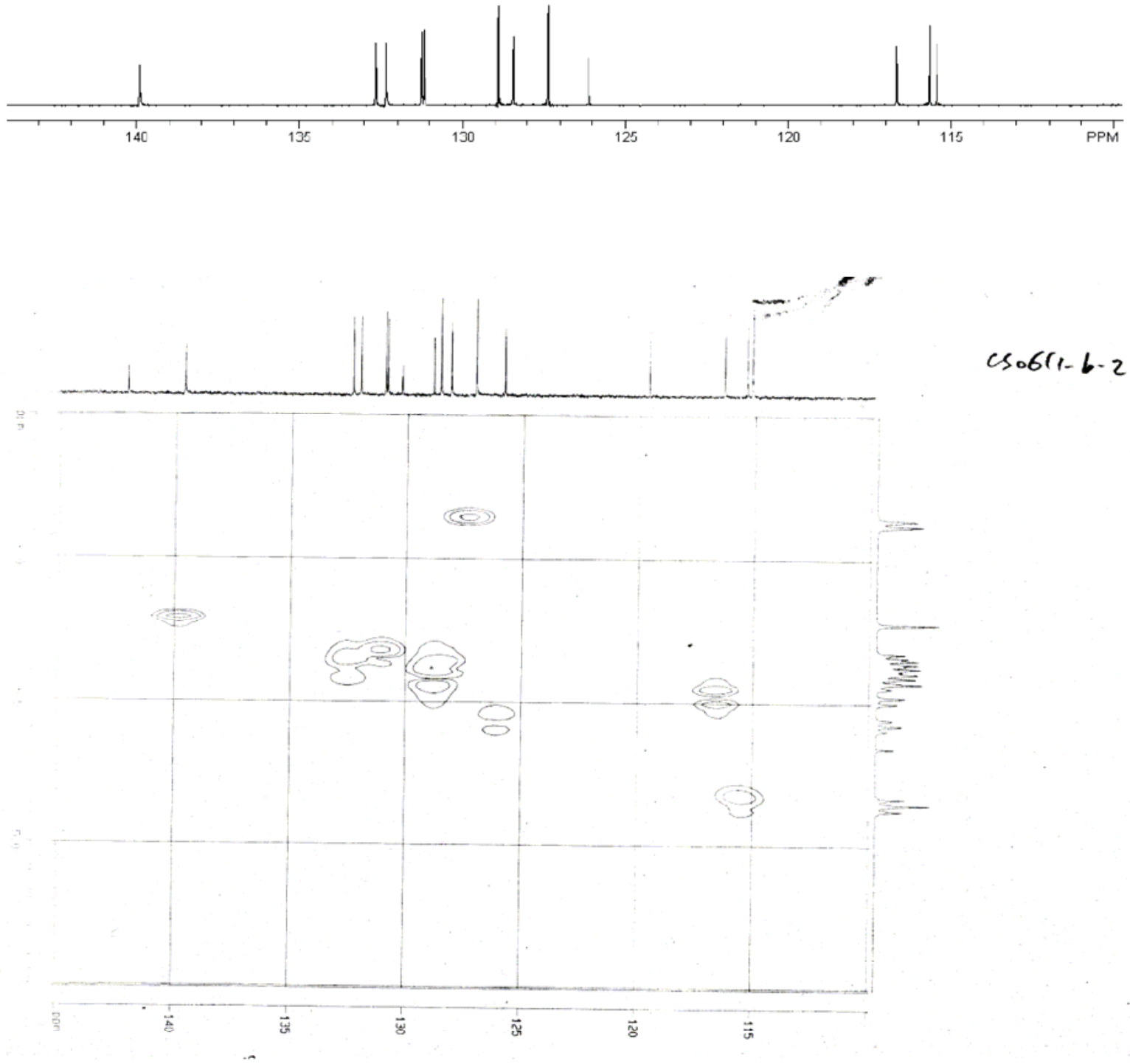

cso6st-b-2 

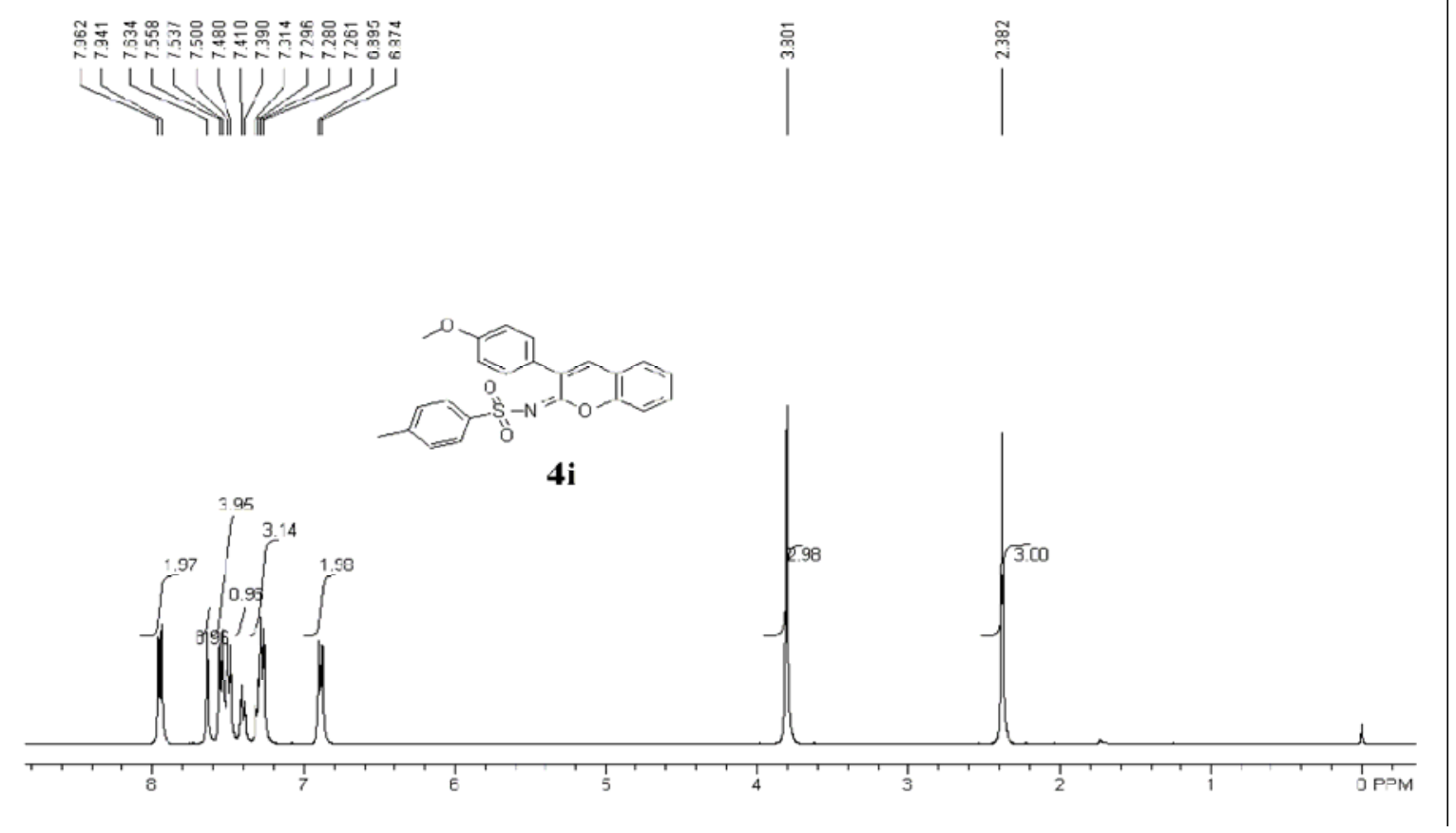

1)

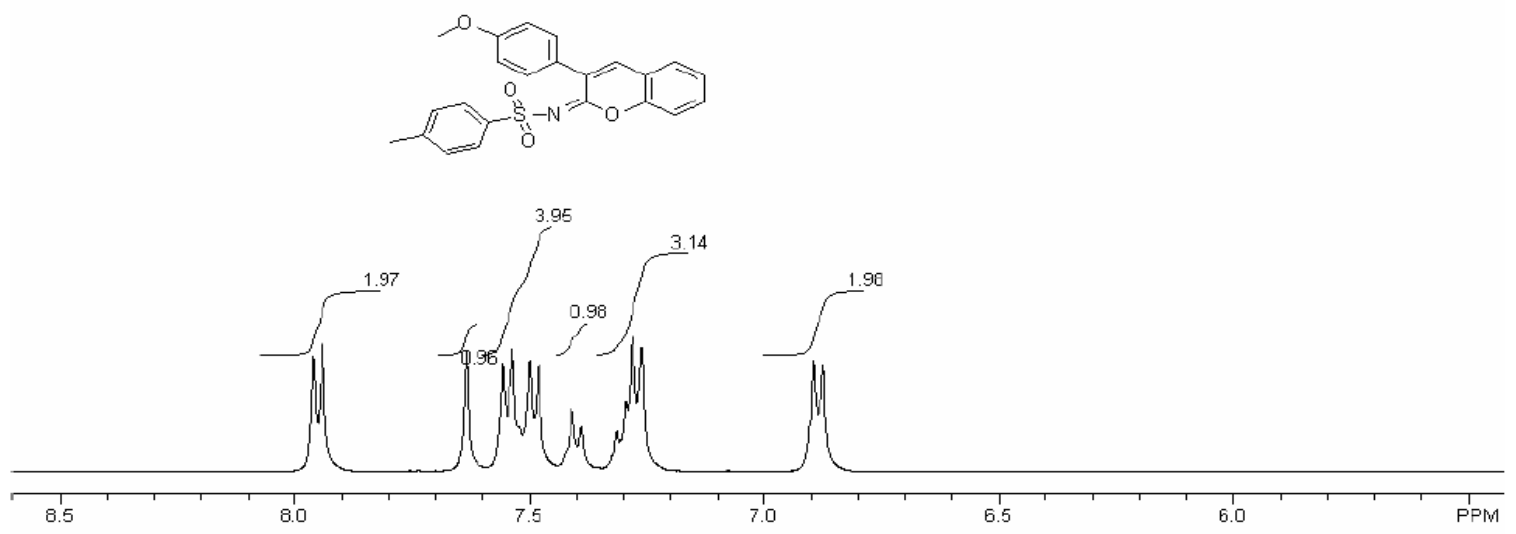



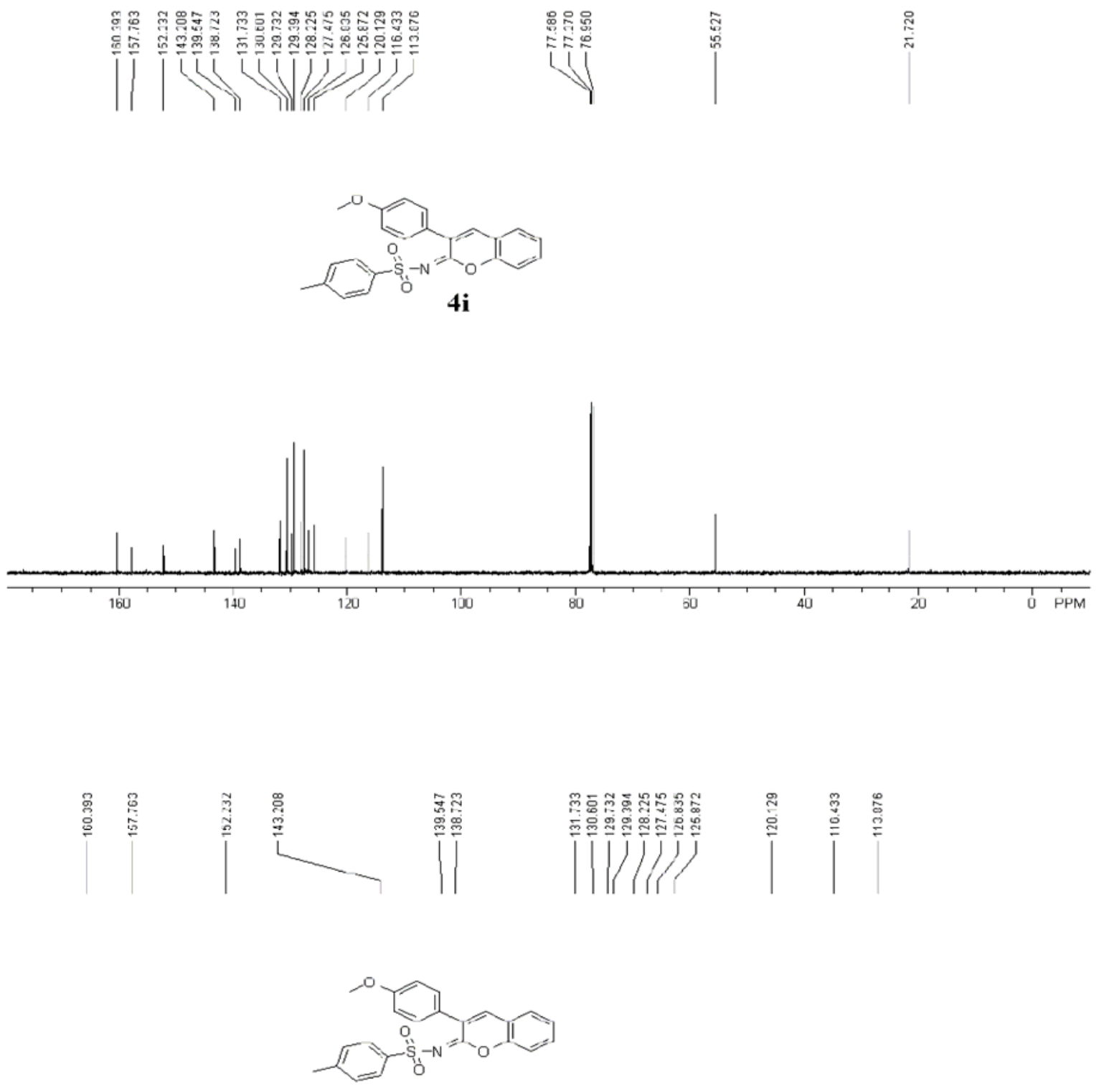

$4 i$

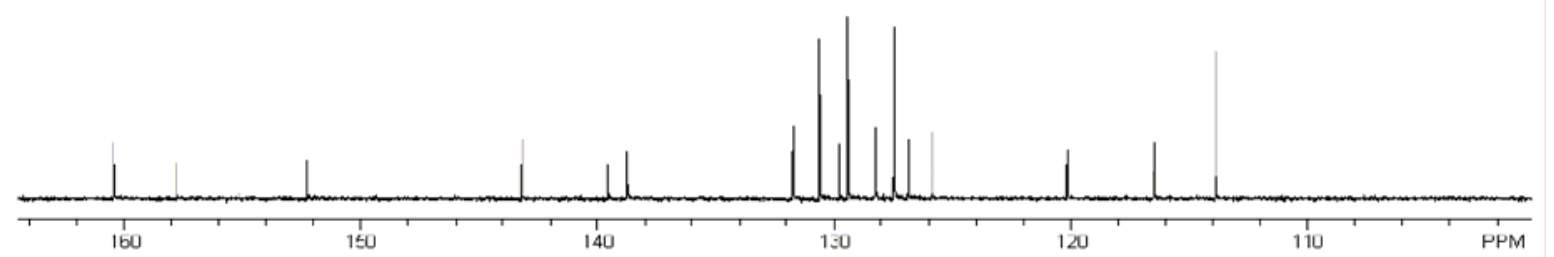



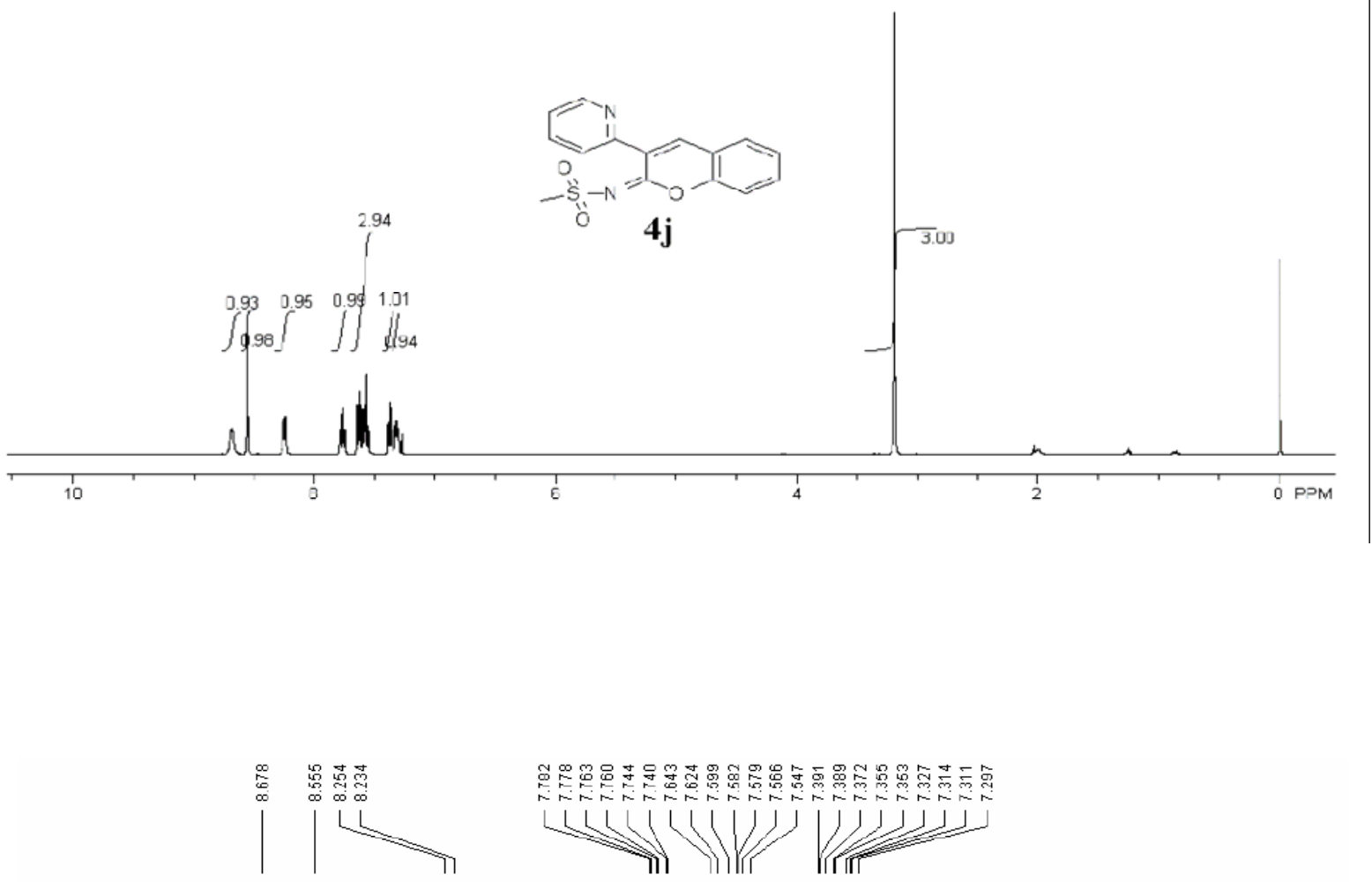

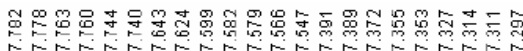

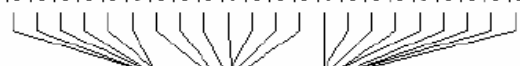

7

iाi

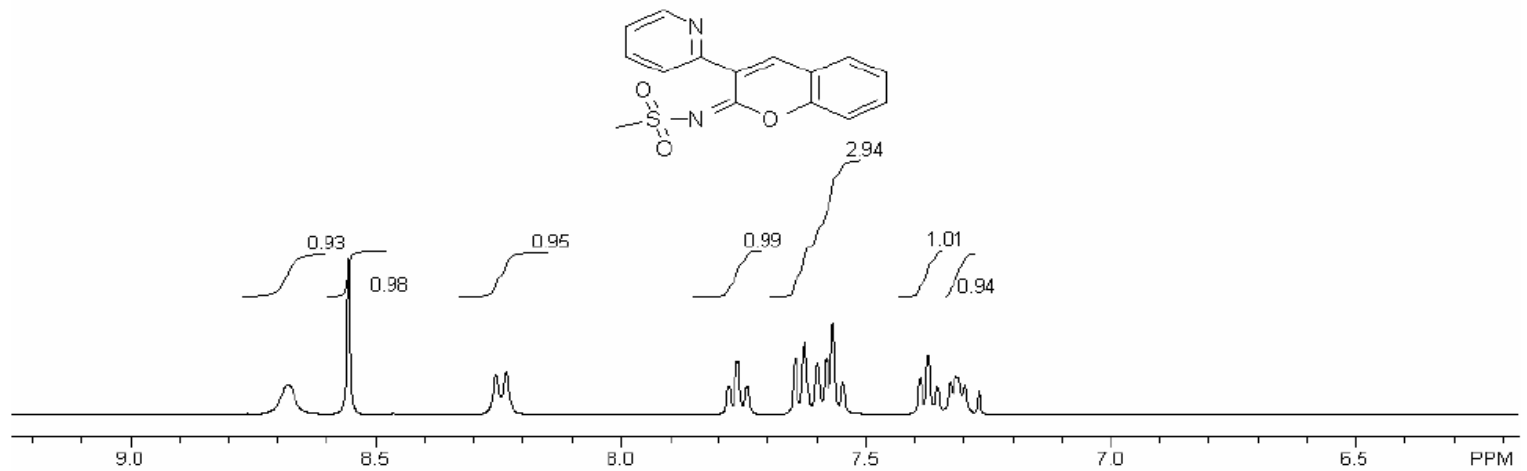


||
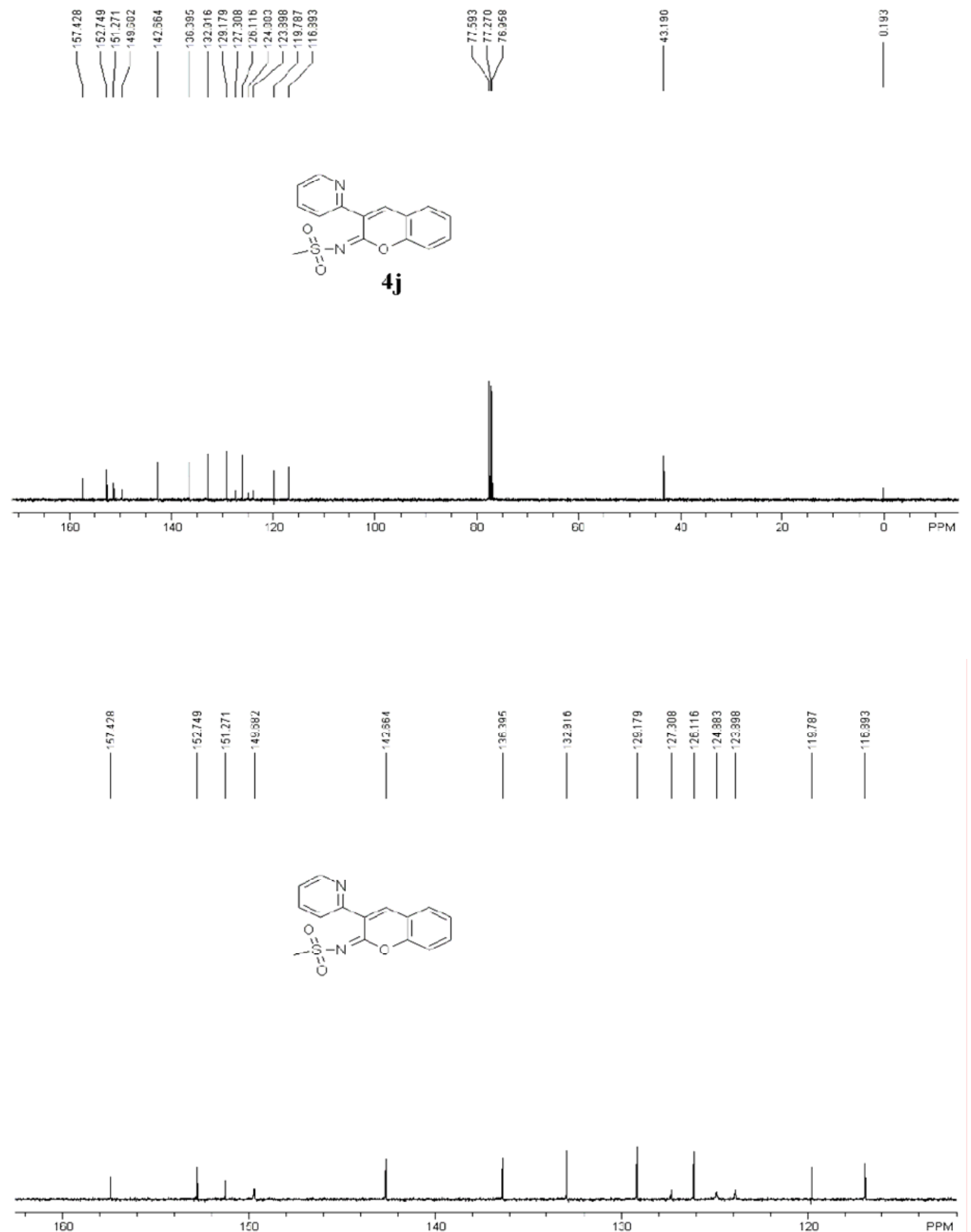

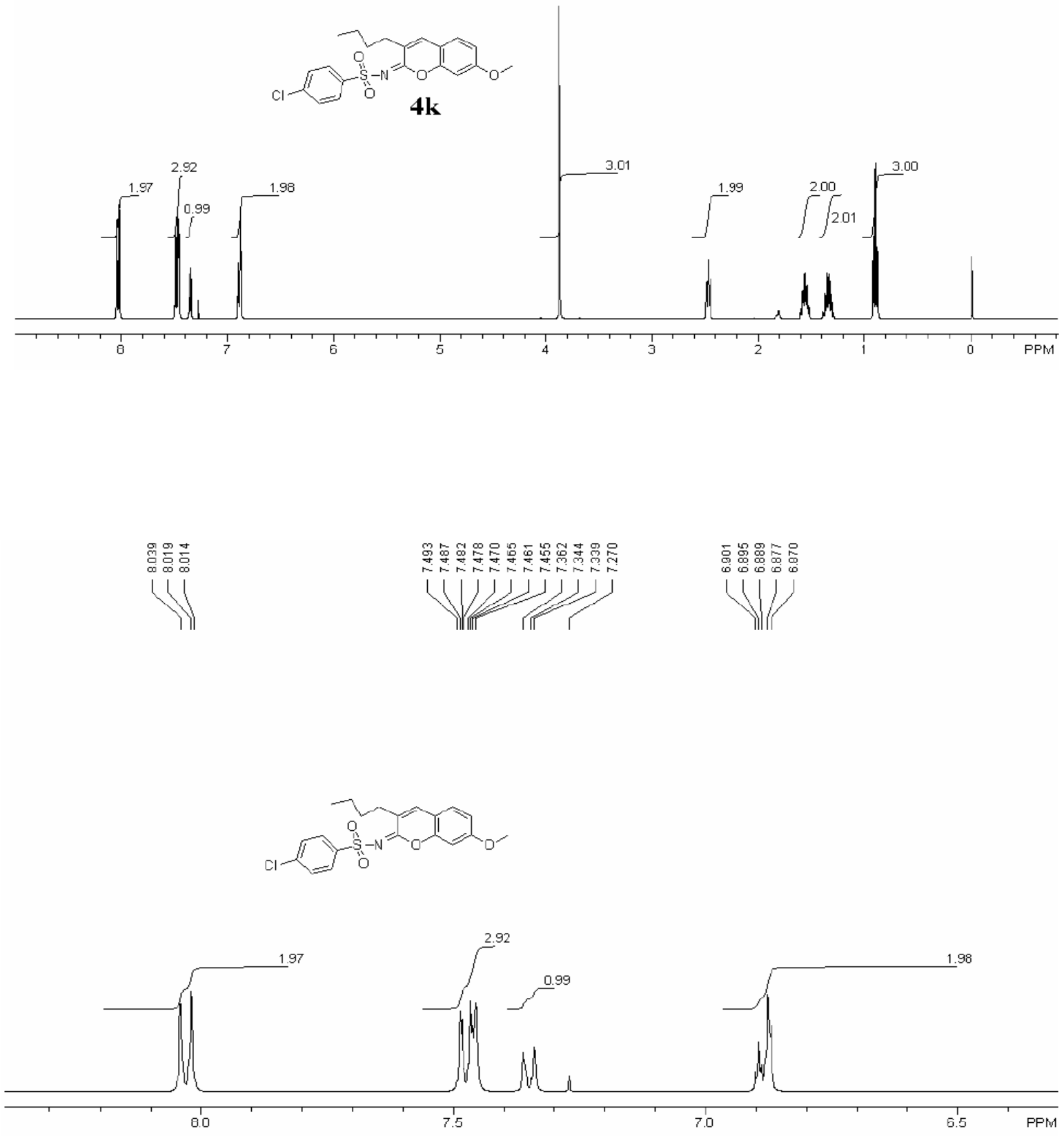


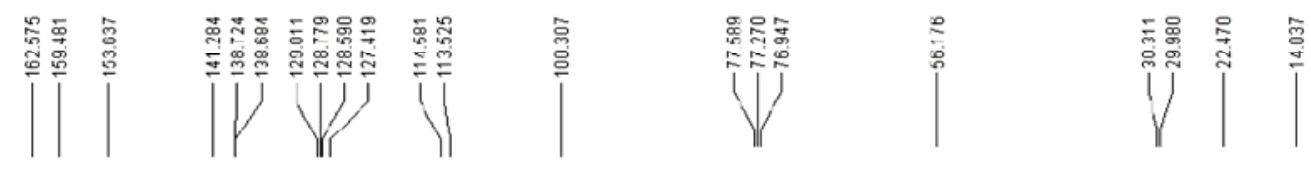

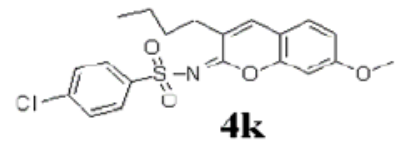

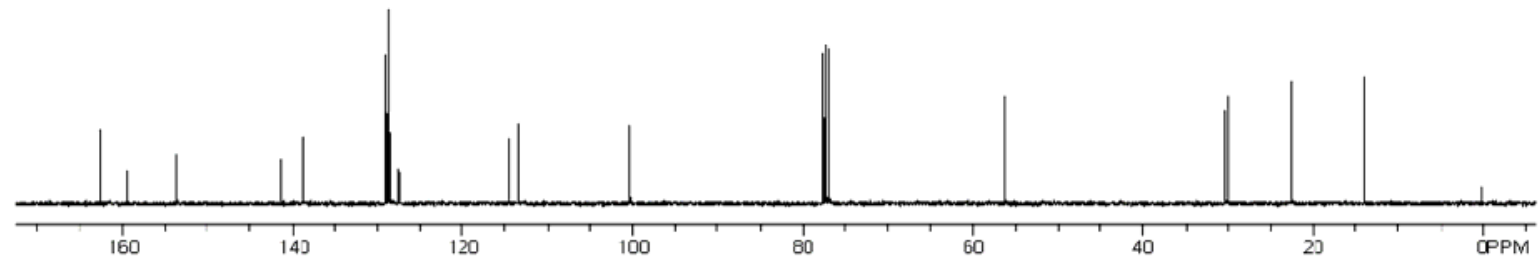

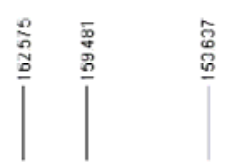

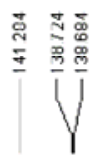

|

$\mid$

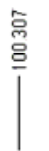

1.

$4 k$

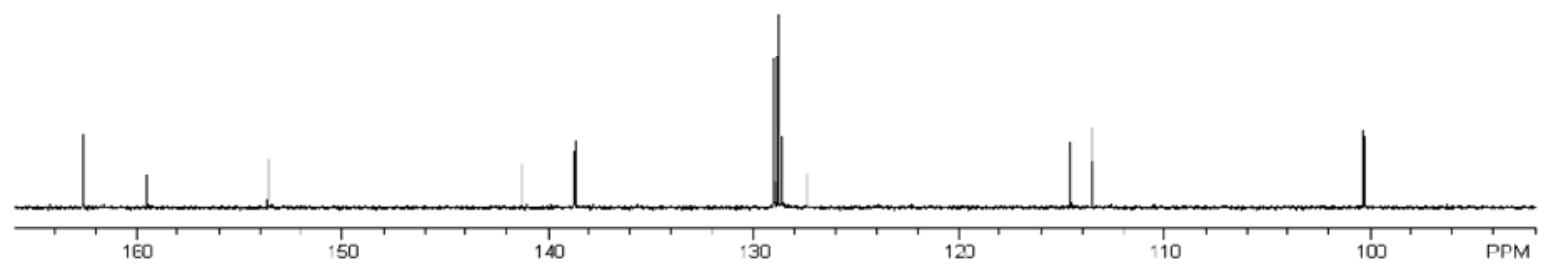



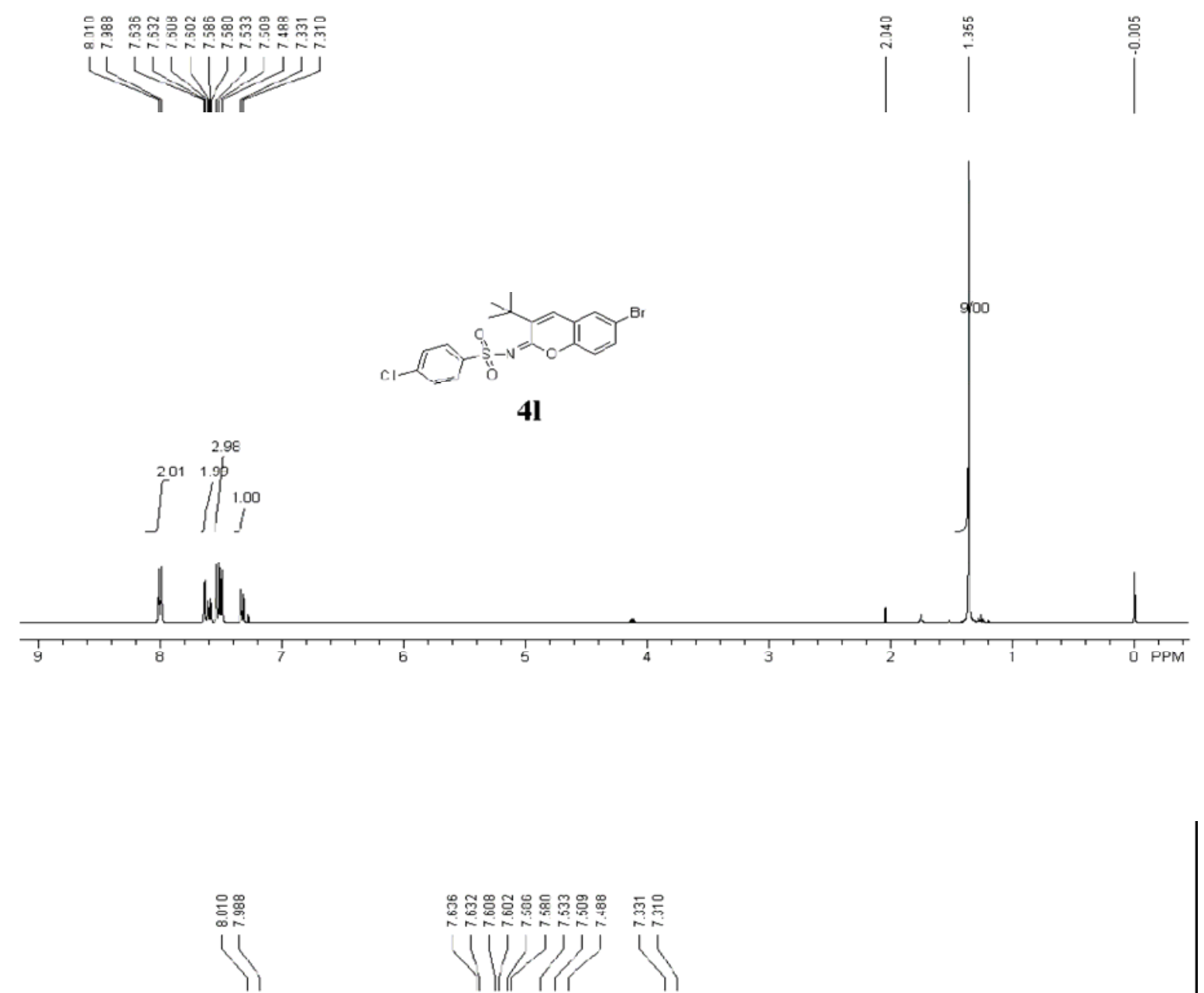

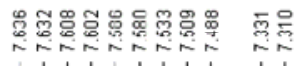

YWIIII

$\overbrace{0}^{B r}$
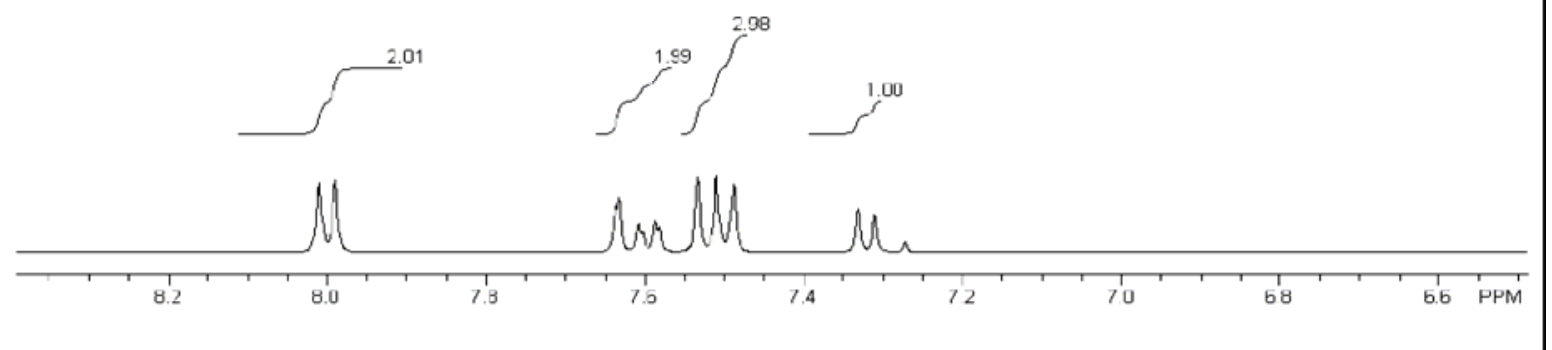

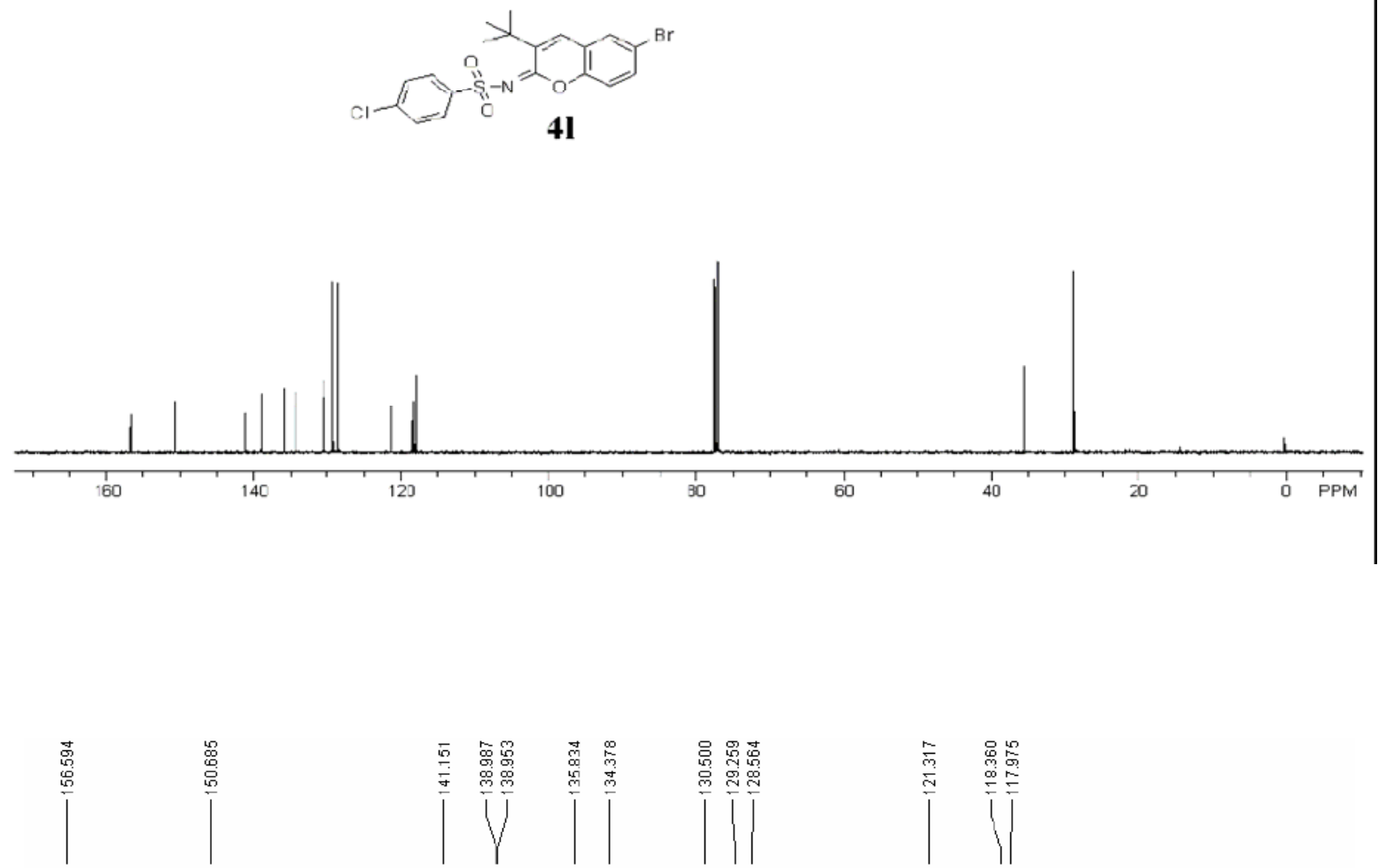

$\overbrace{\mathrm{O}}^{\mathrm{C}} \mathrm{Cr}$

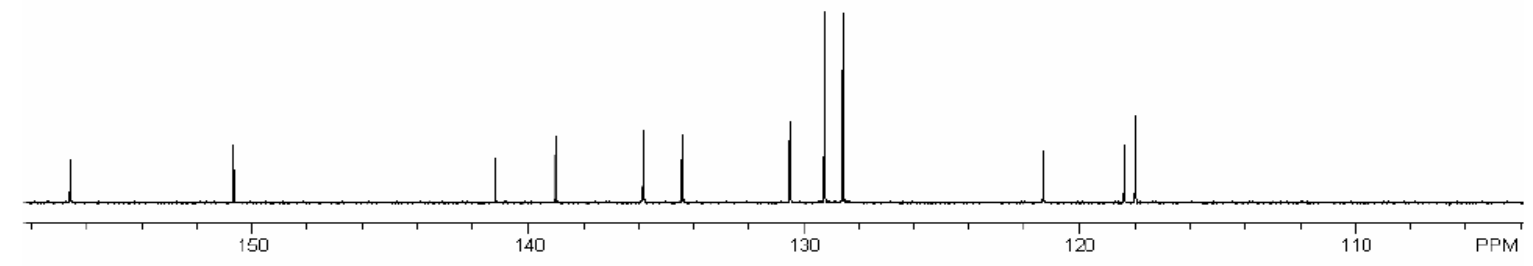




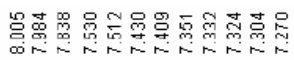

LLLLLLLIII)

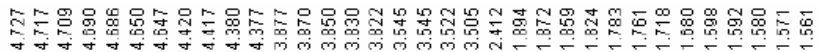

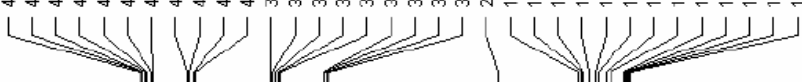

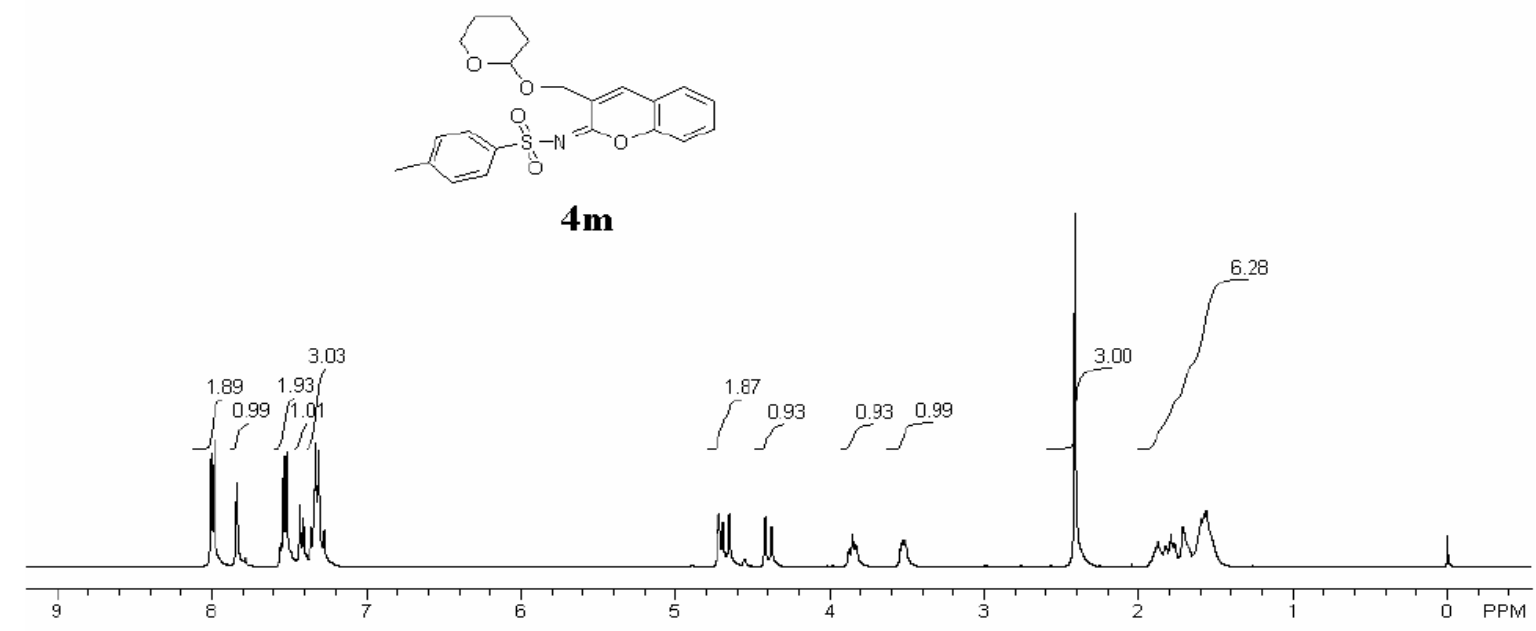

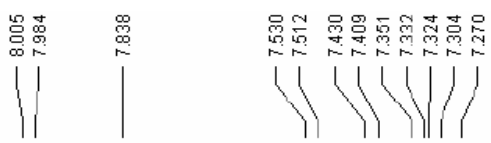
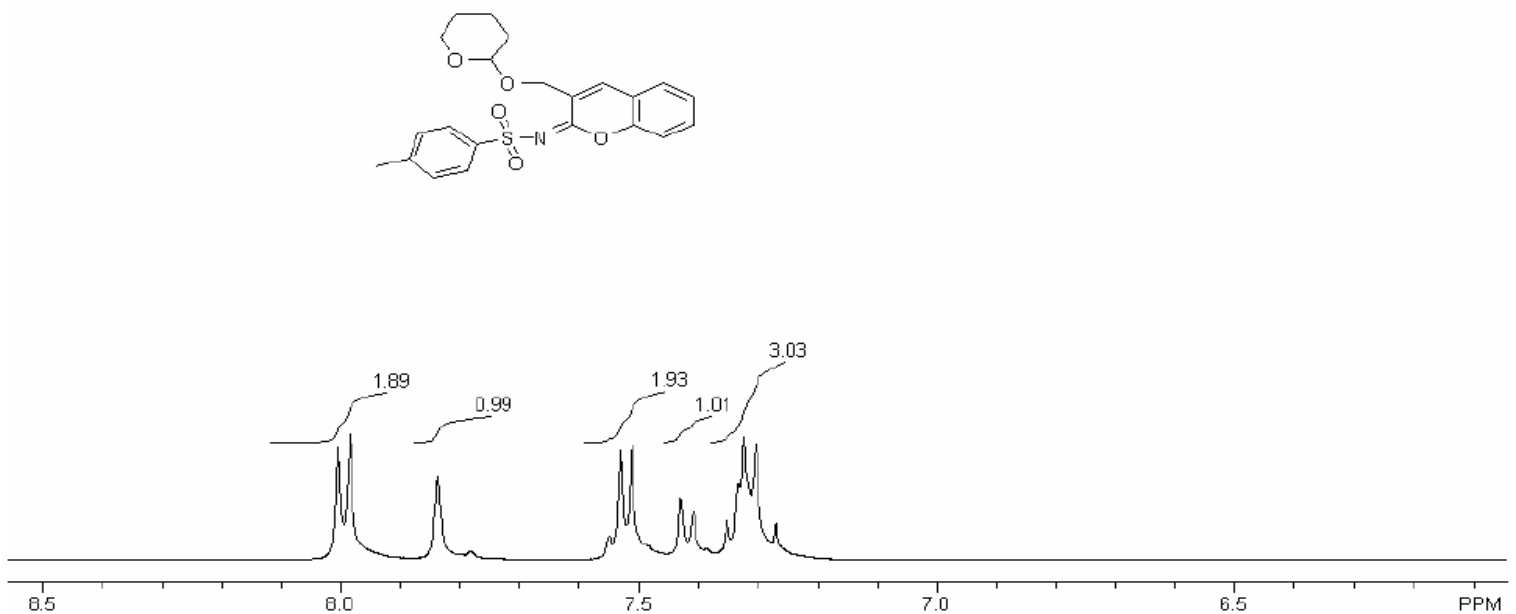

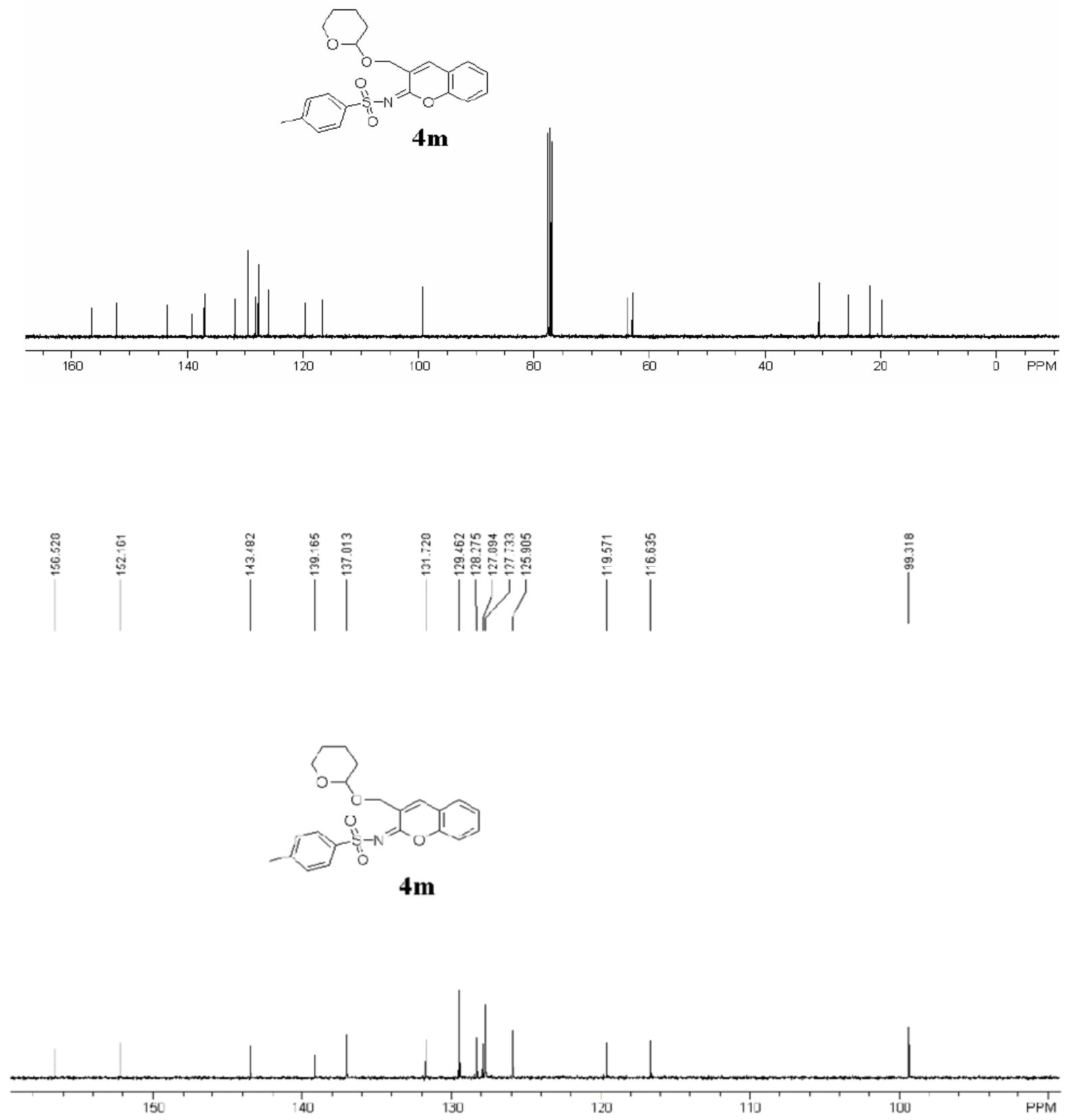


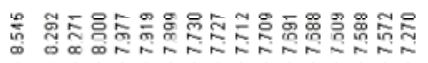

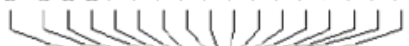

ᄀํㅔ는
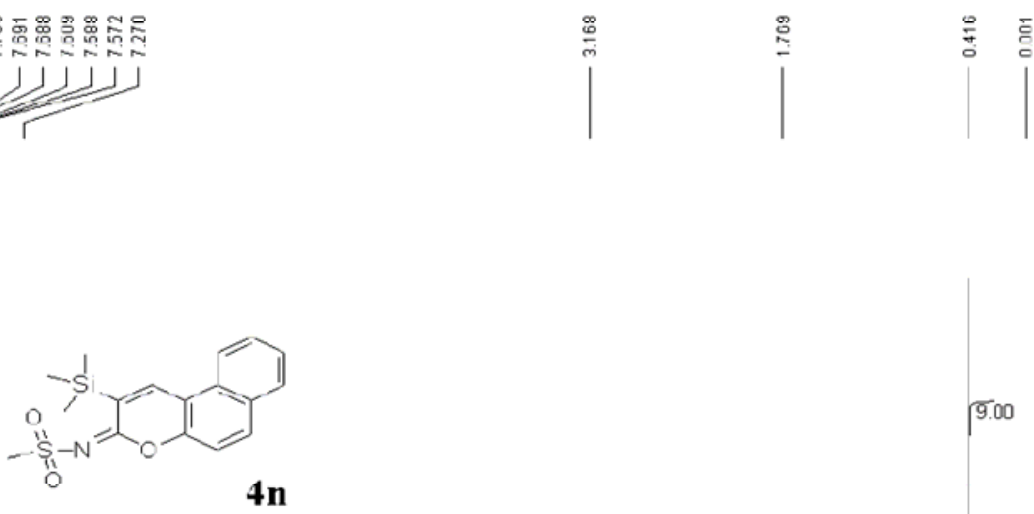

4n

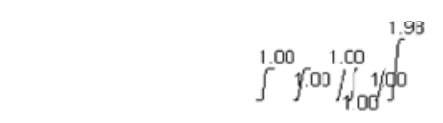

, li d

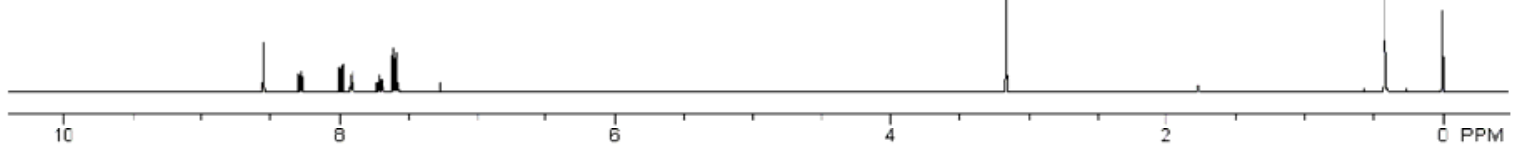

!<smiles>C[Si](C)(C)C1=Cc2c(ccc3ccccc23)OC1N=S(C)(C)=O</smiles>

$\sqrt{1.00} \sqrt{1.00}$

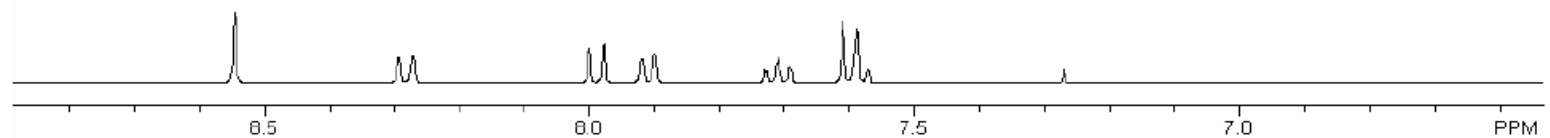



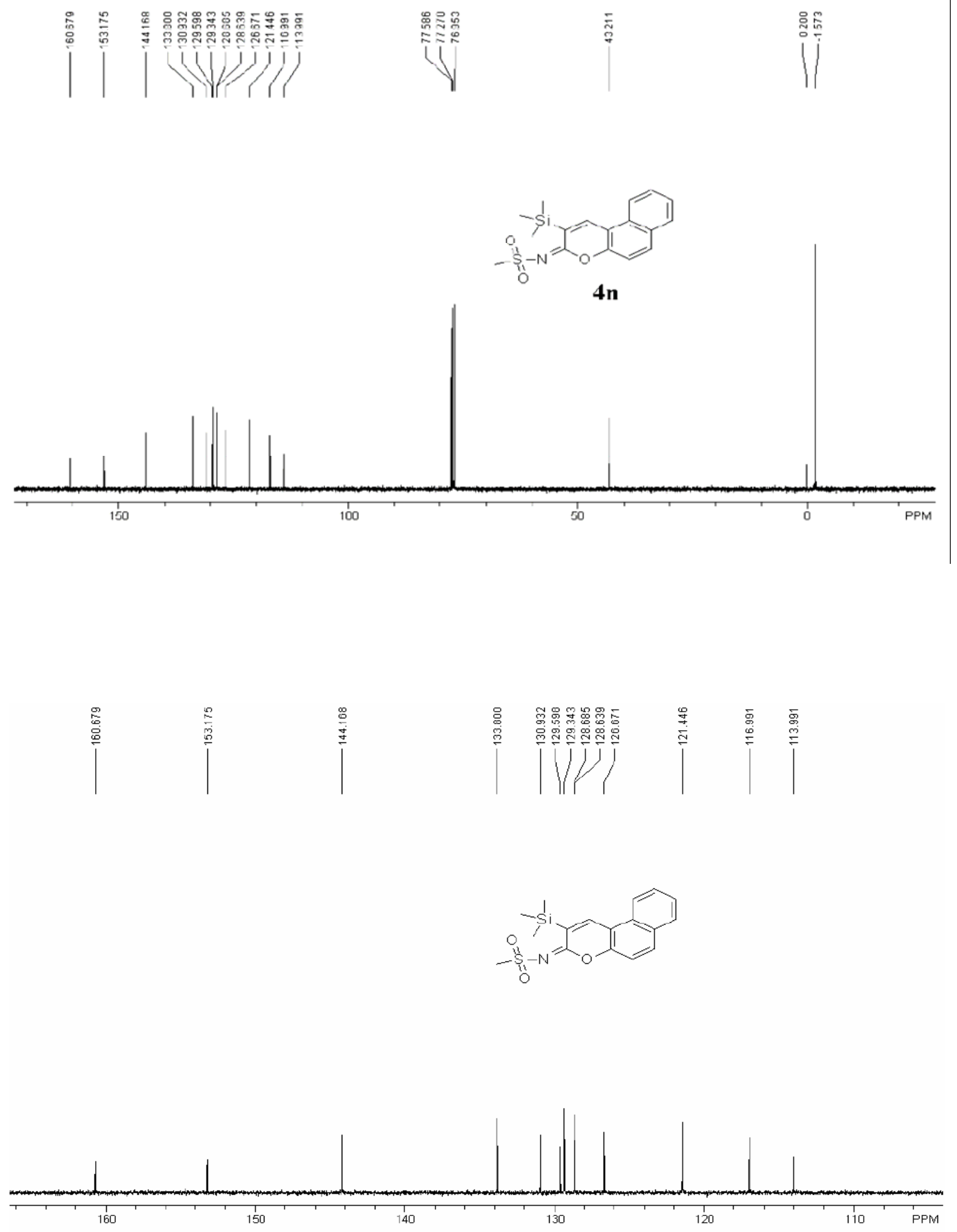


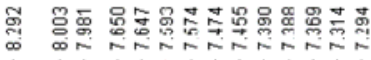

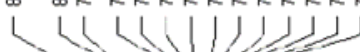

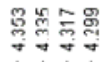

LI)

i
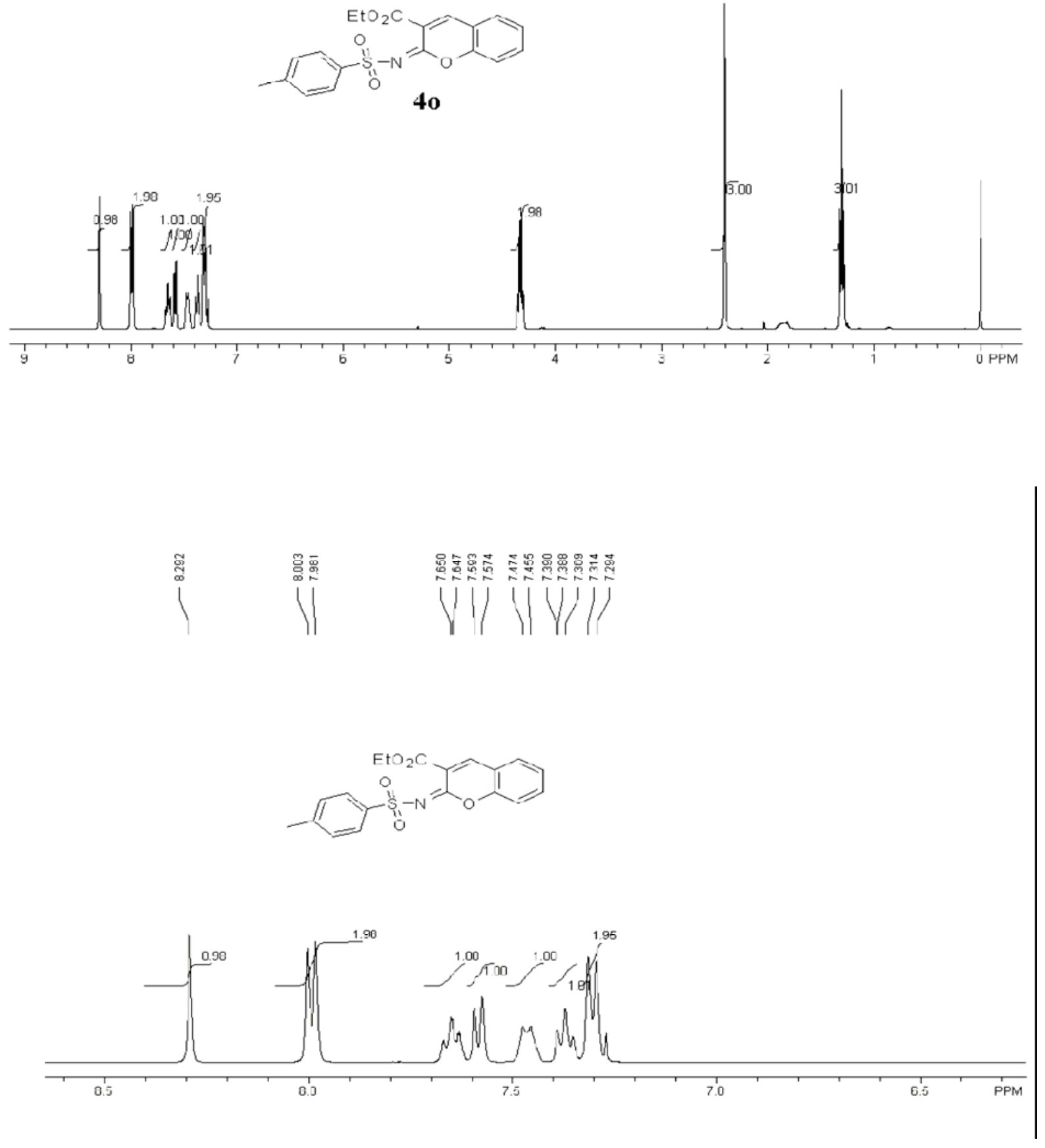

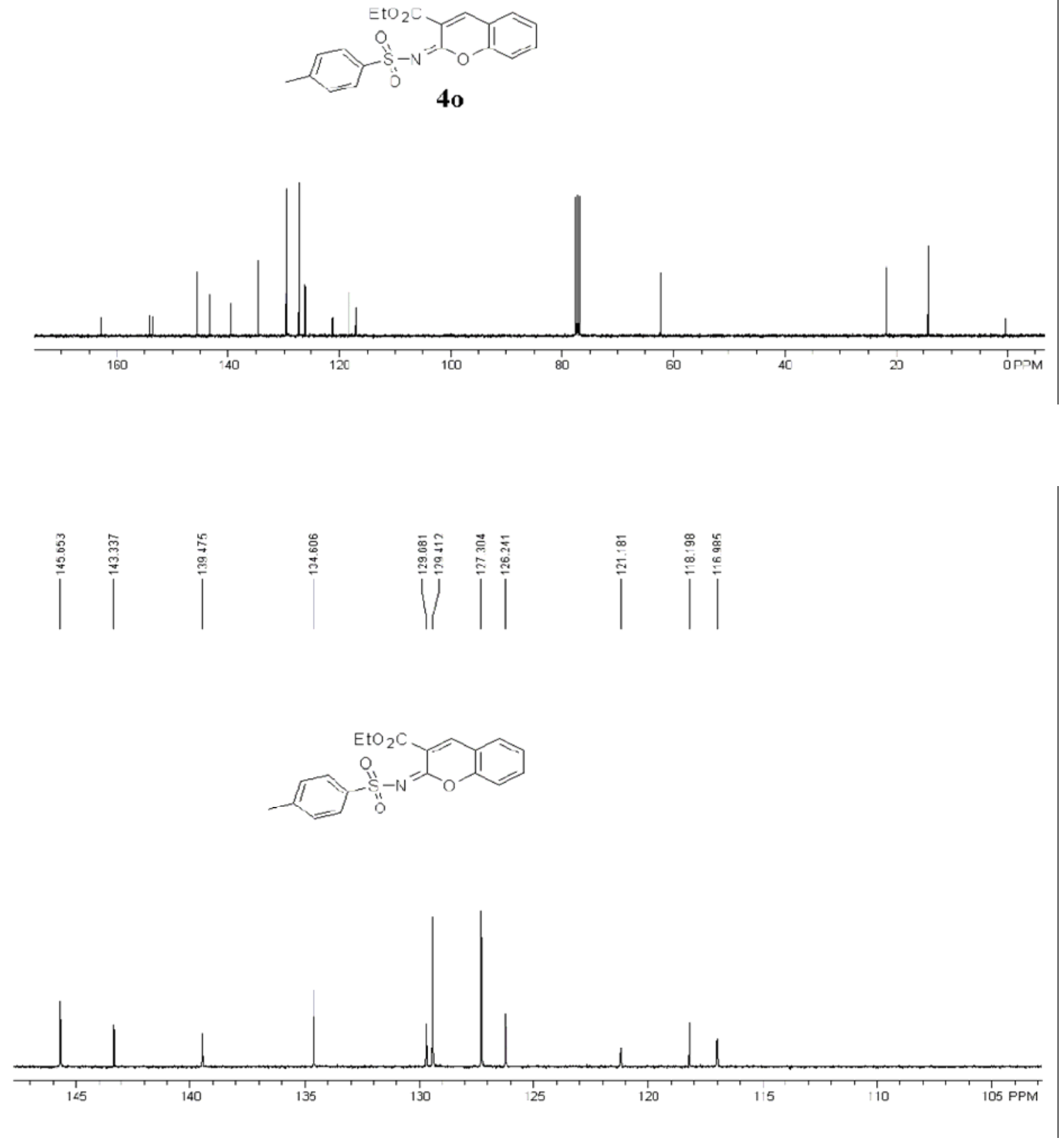


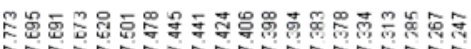

$L L L L L(L) \mid J J J j\rfloor J$

> Thin
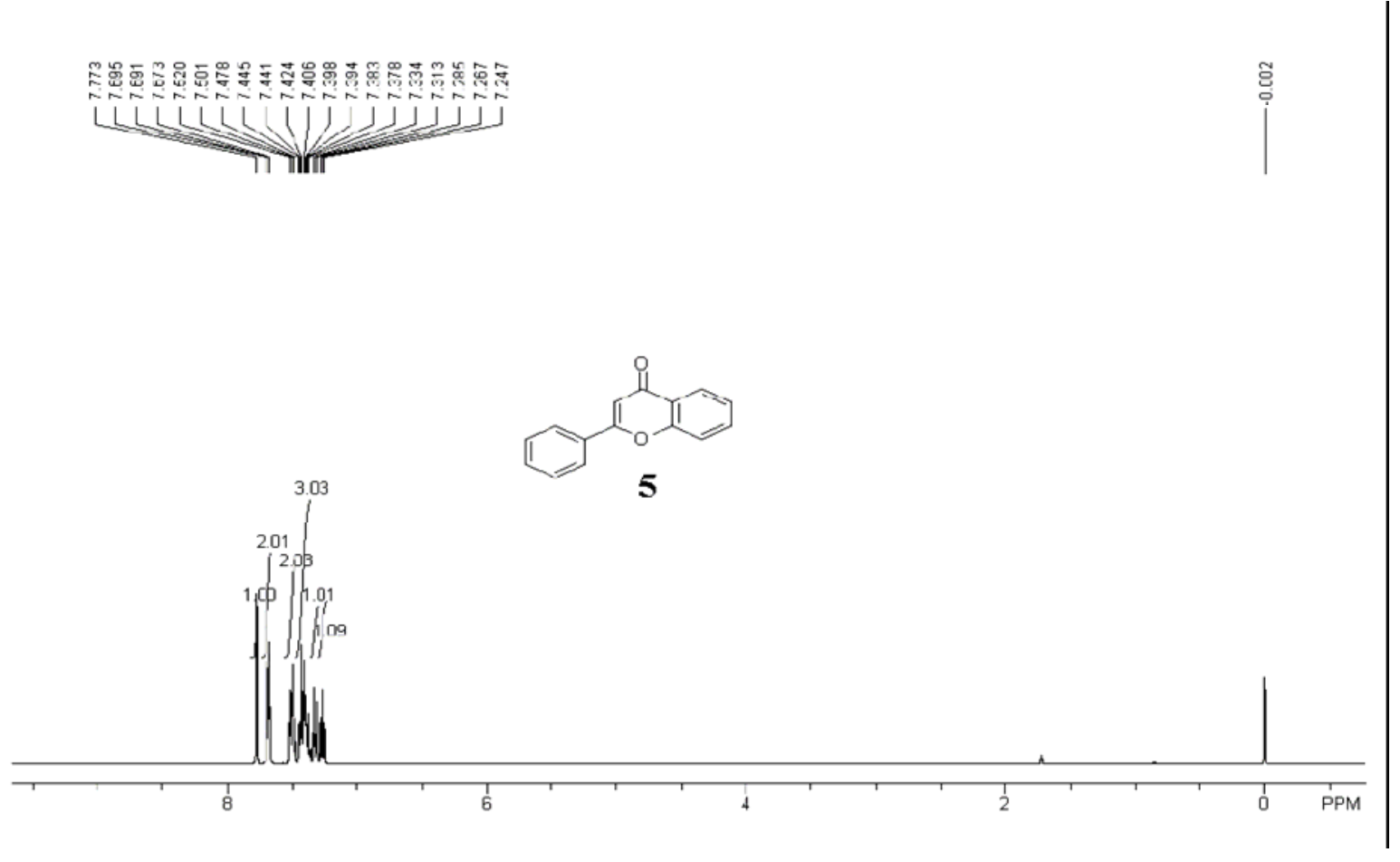

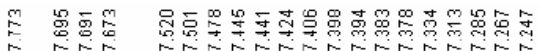

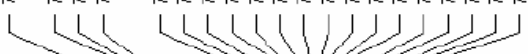

(7) 71 गो

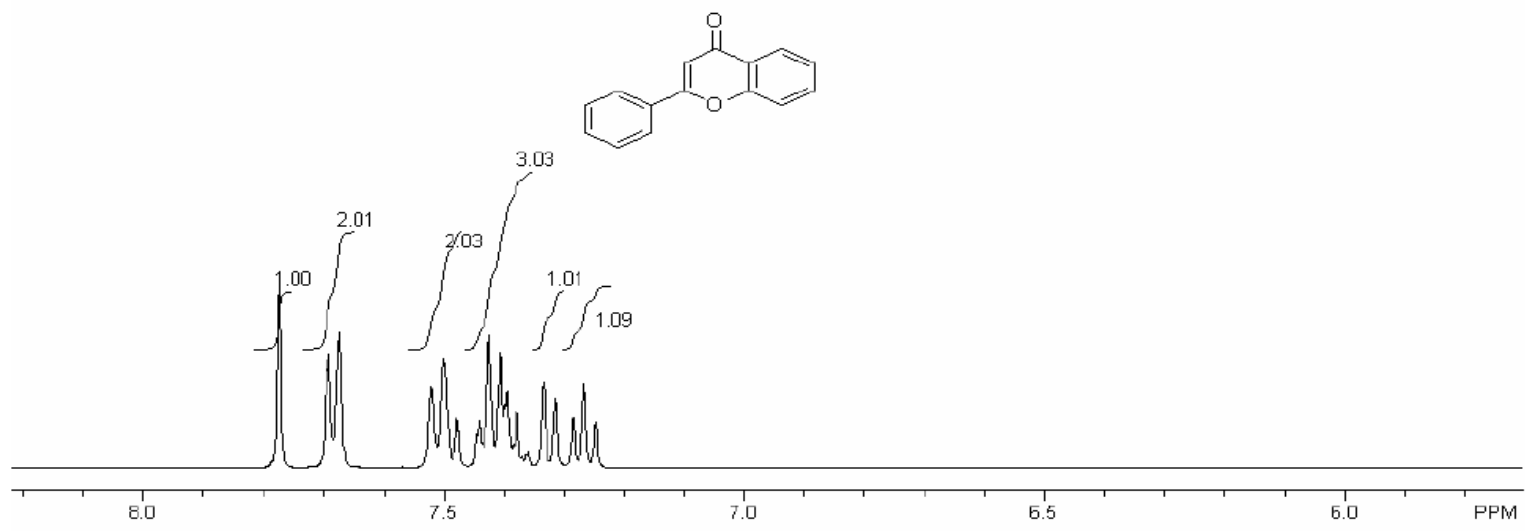



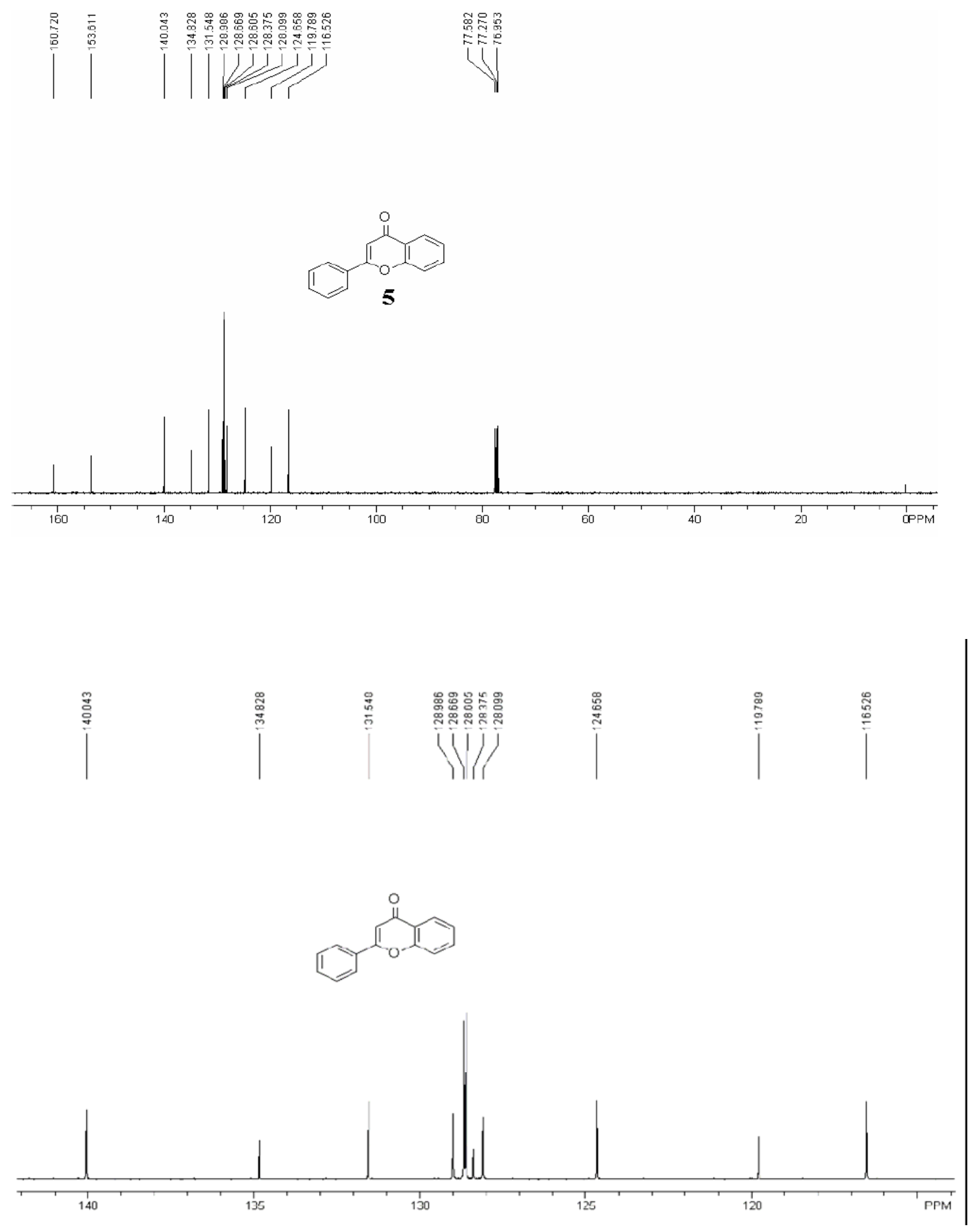


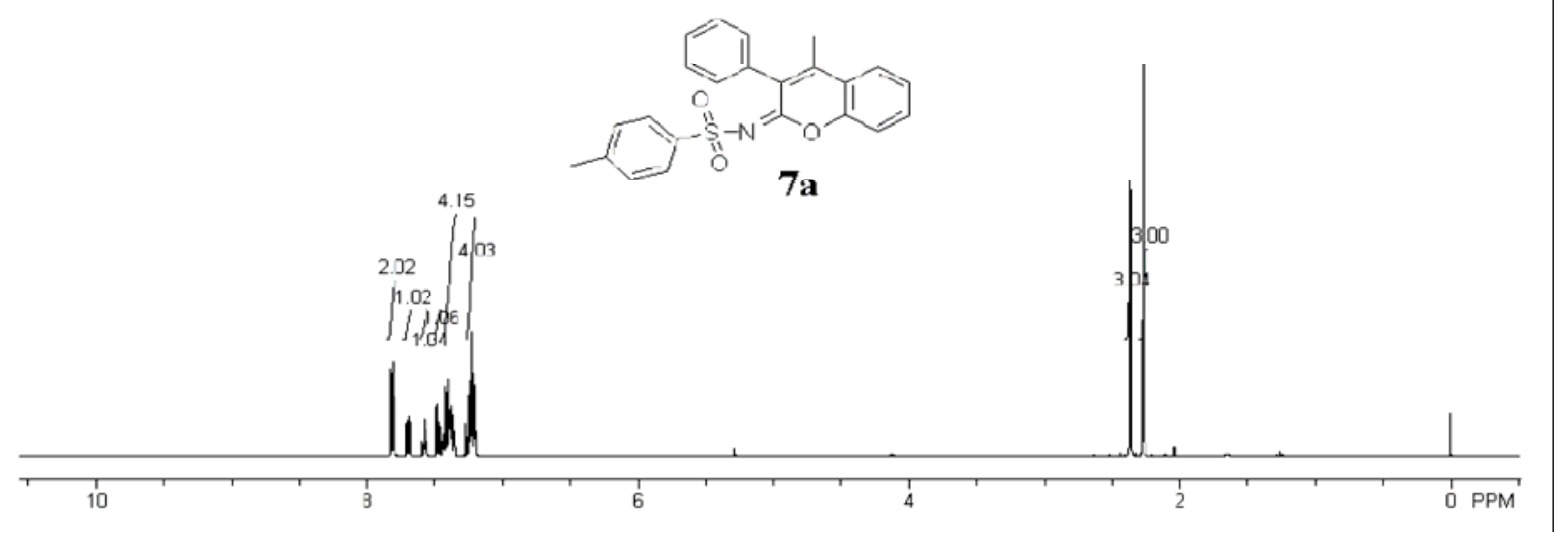

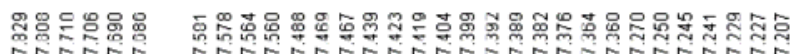

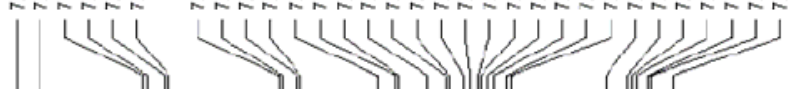

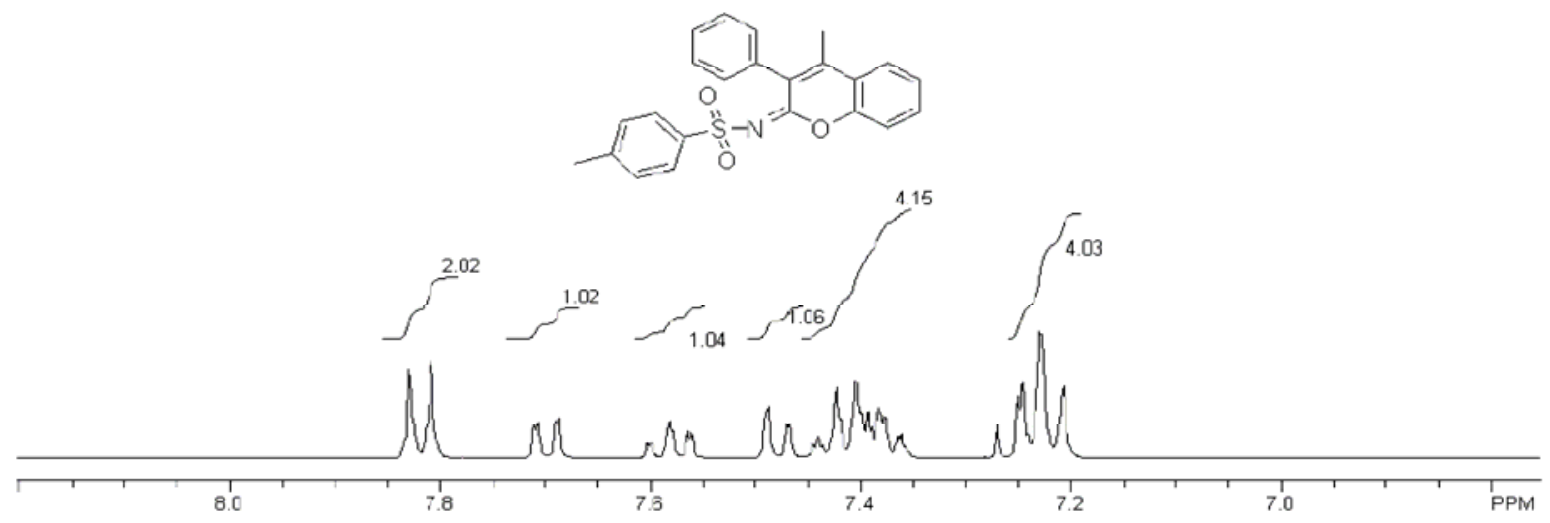



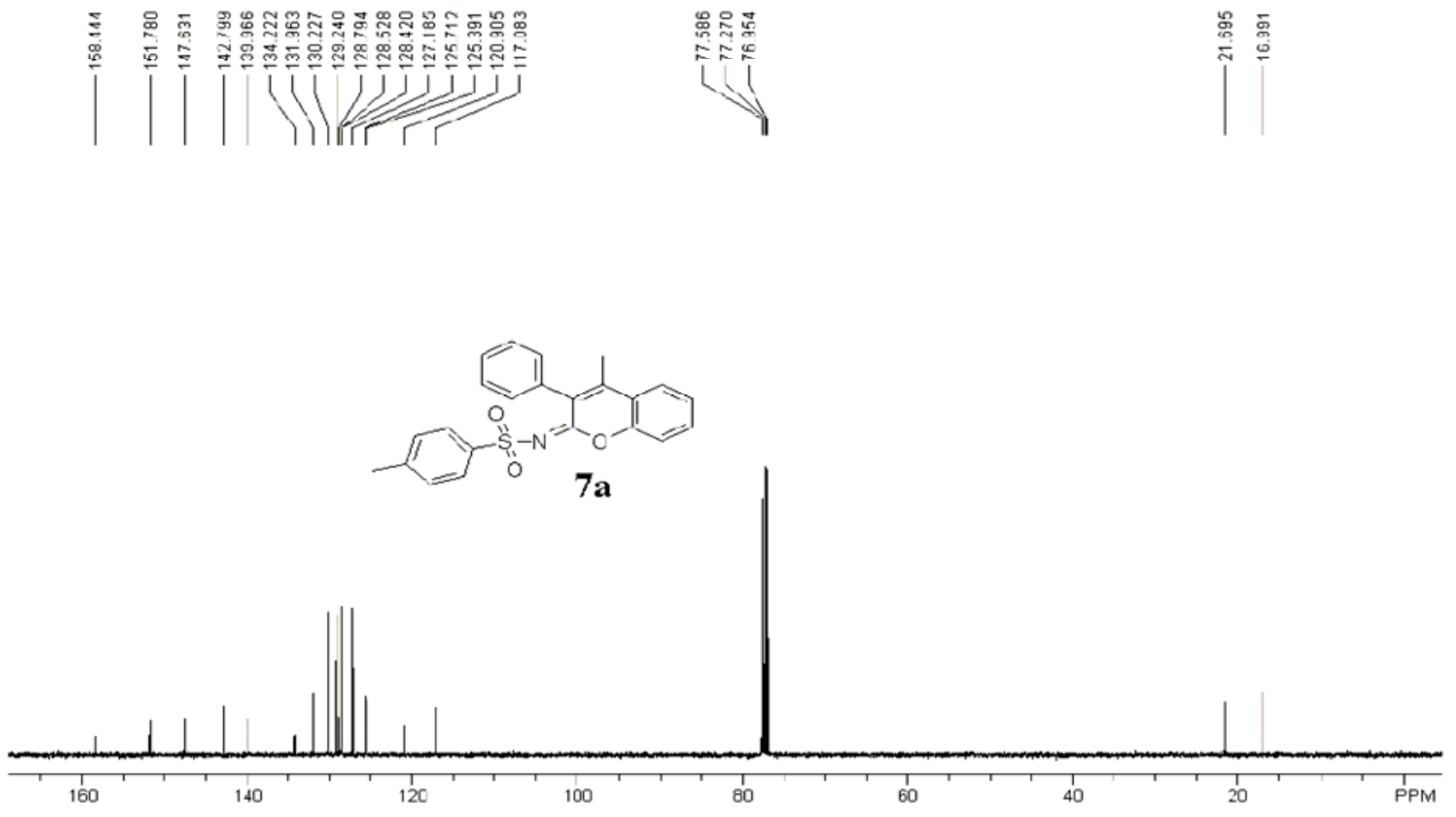

$\left.\right|^{\frac{1}{ \pm 0}}$<smiles>Cc1ccc(S(=O)(=O)N=c2oc3ccccc3c(C)c2-c2ccccc2)cc1</smiles>

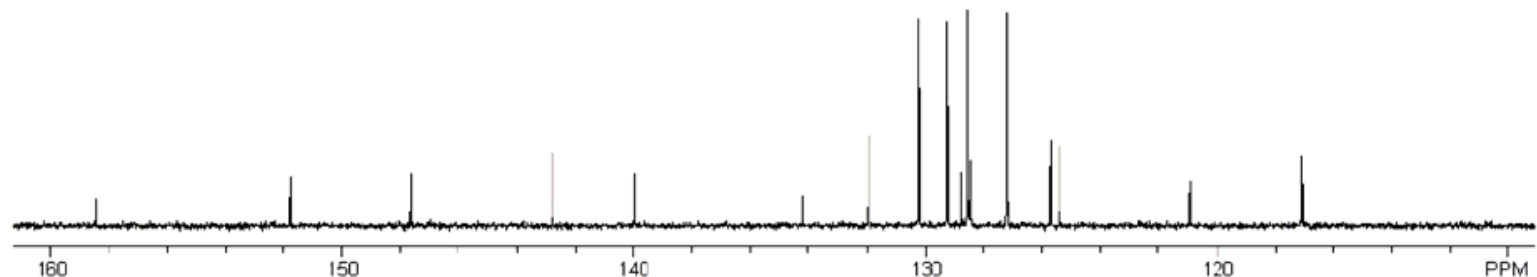




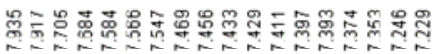

LMUUUJJIJ

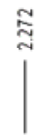

$\stackrel{\circ}{i}$

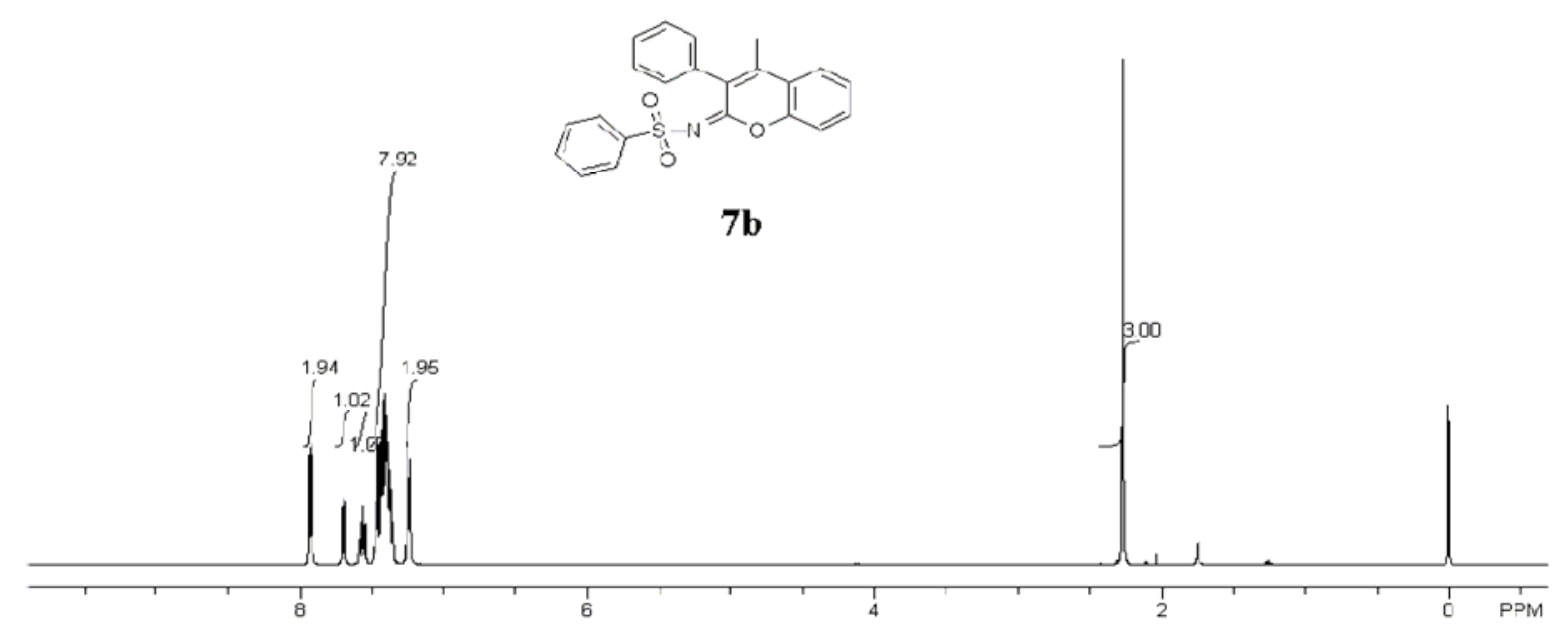

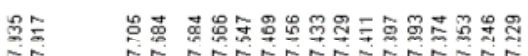

il

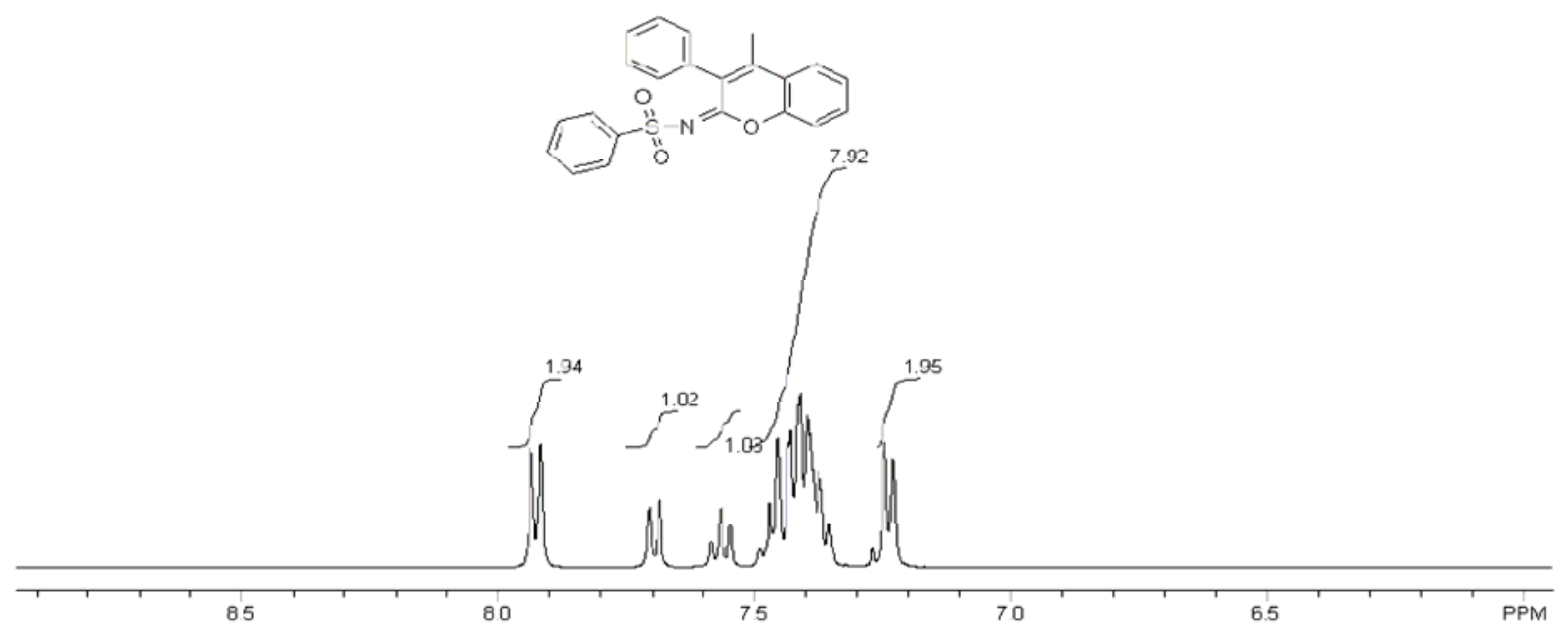



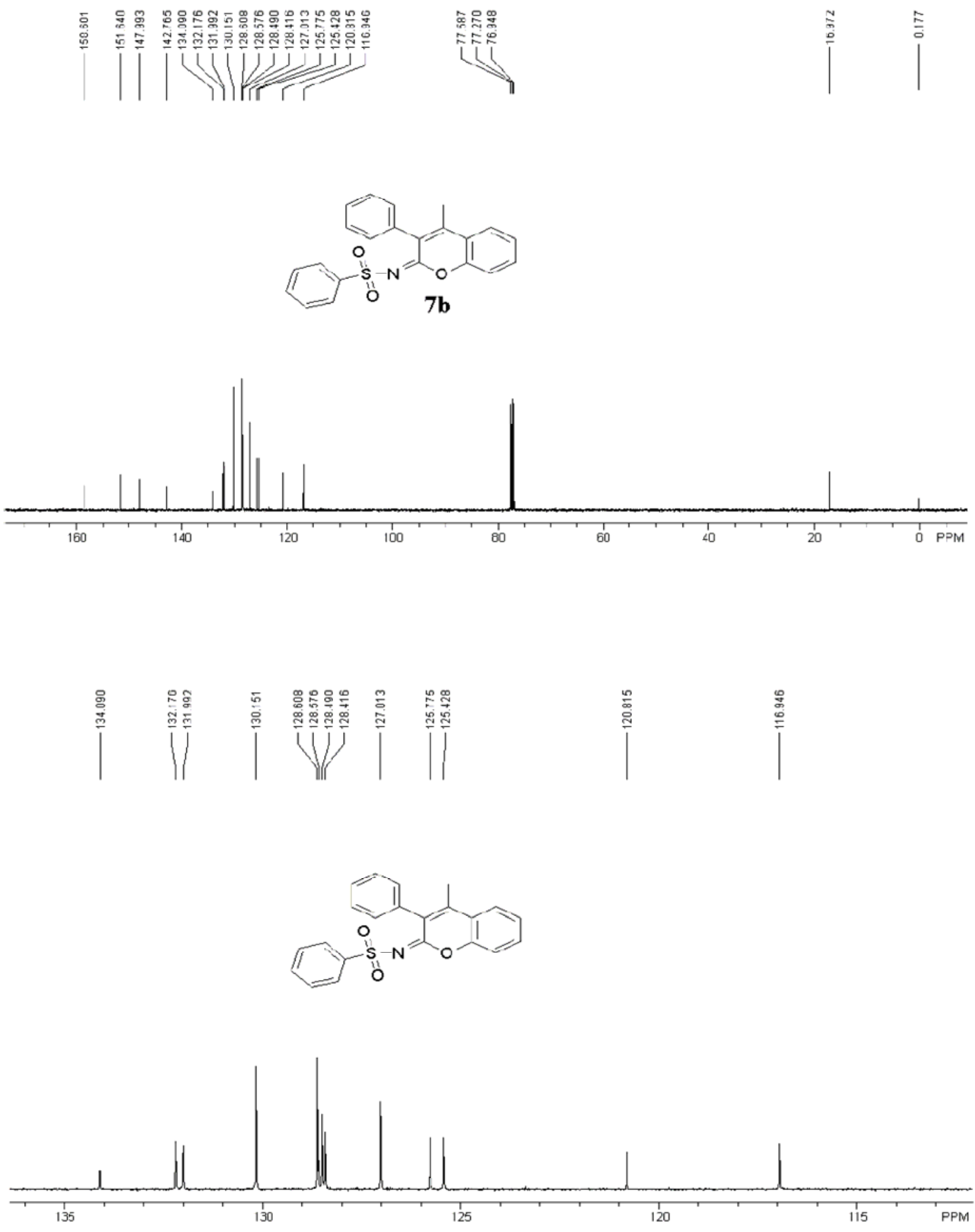


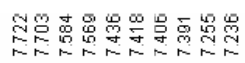

L1)

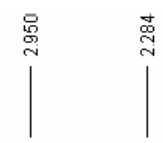

$\int_{1.09}^{4.09} 208$

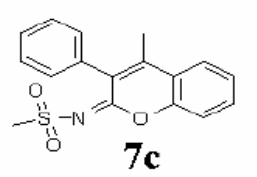

$1.01 \int^{2.08}$

$7 c$

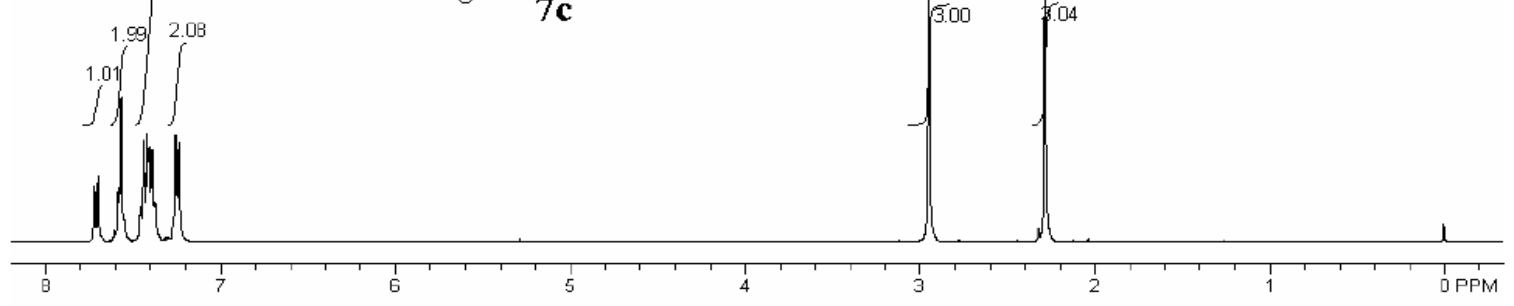

门
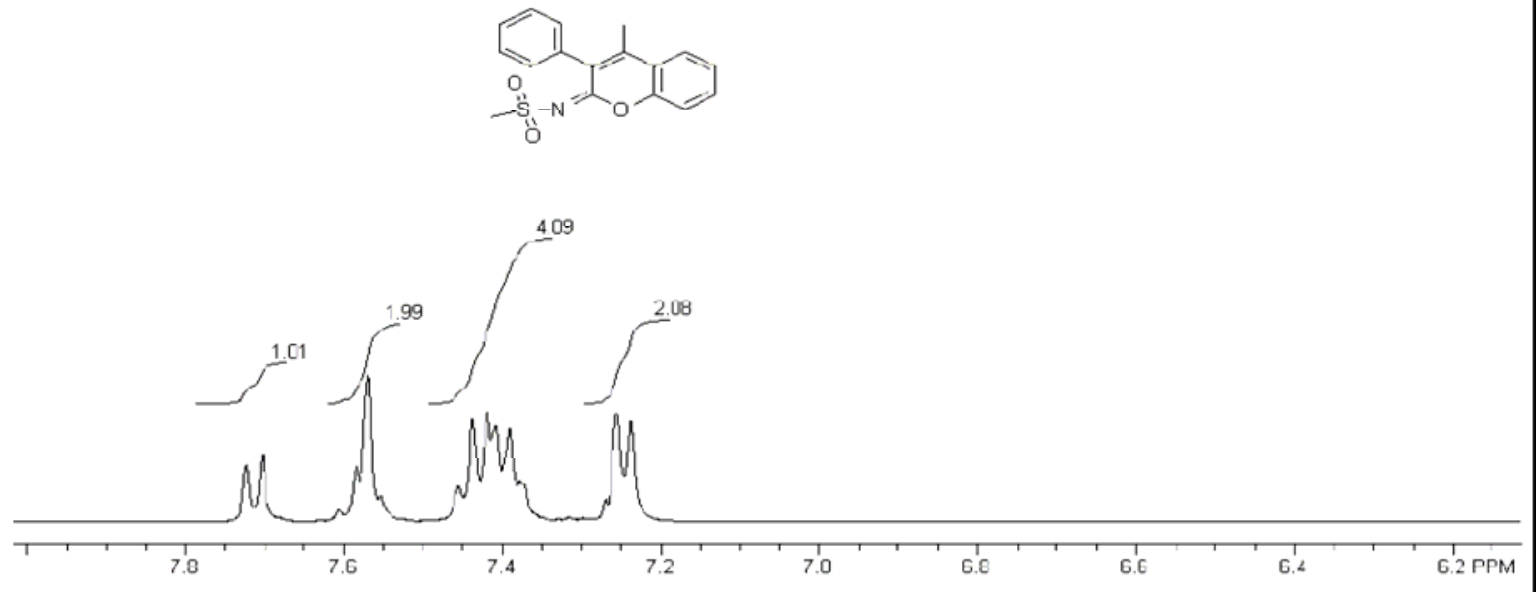

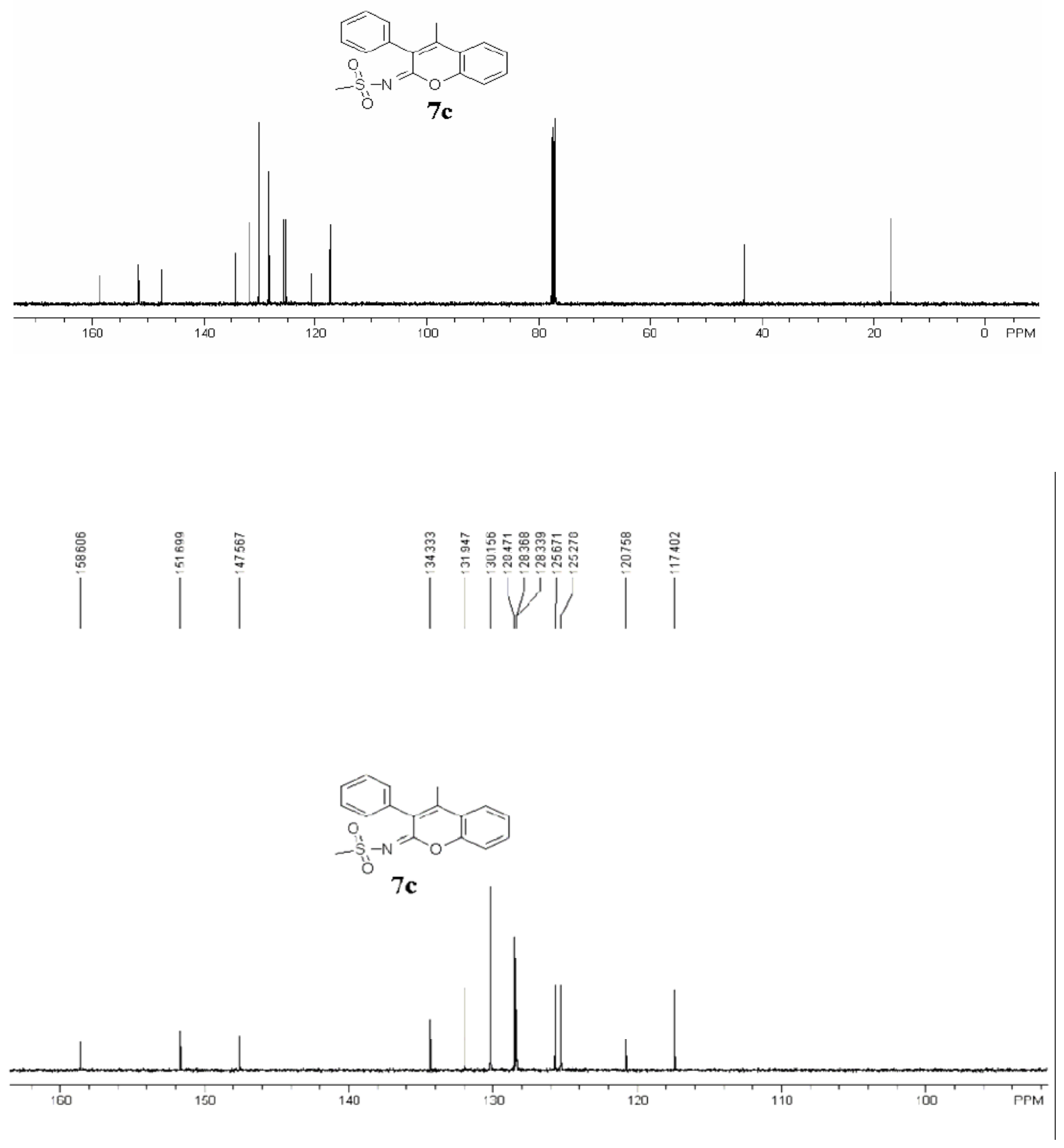

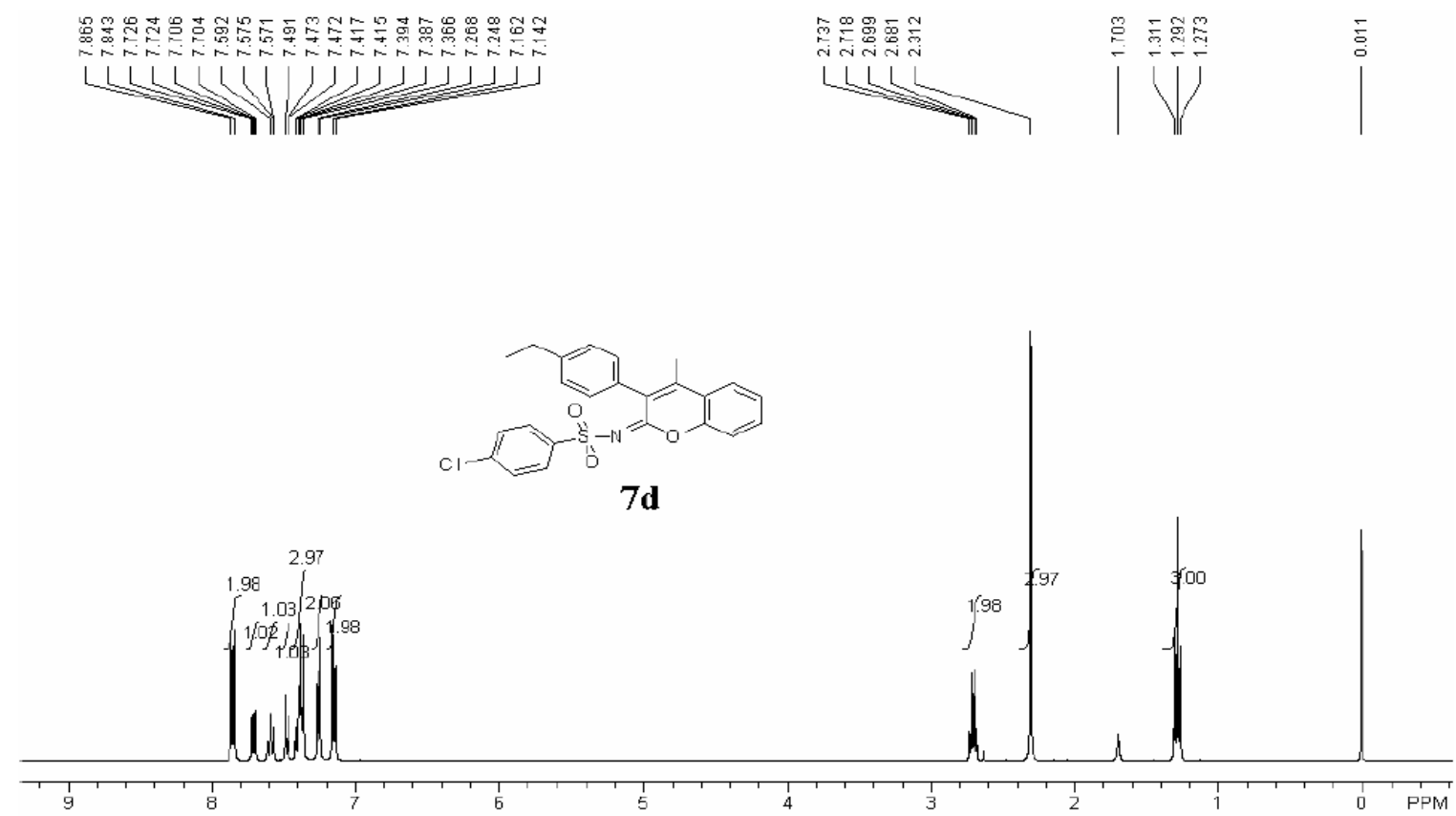

川
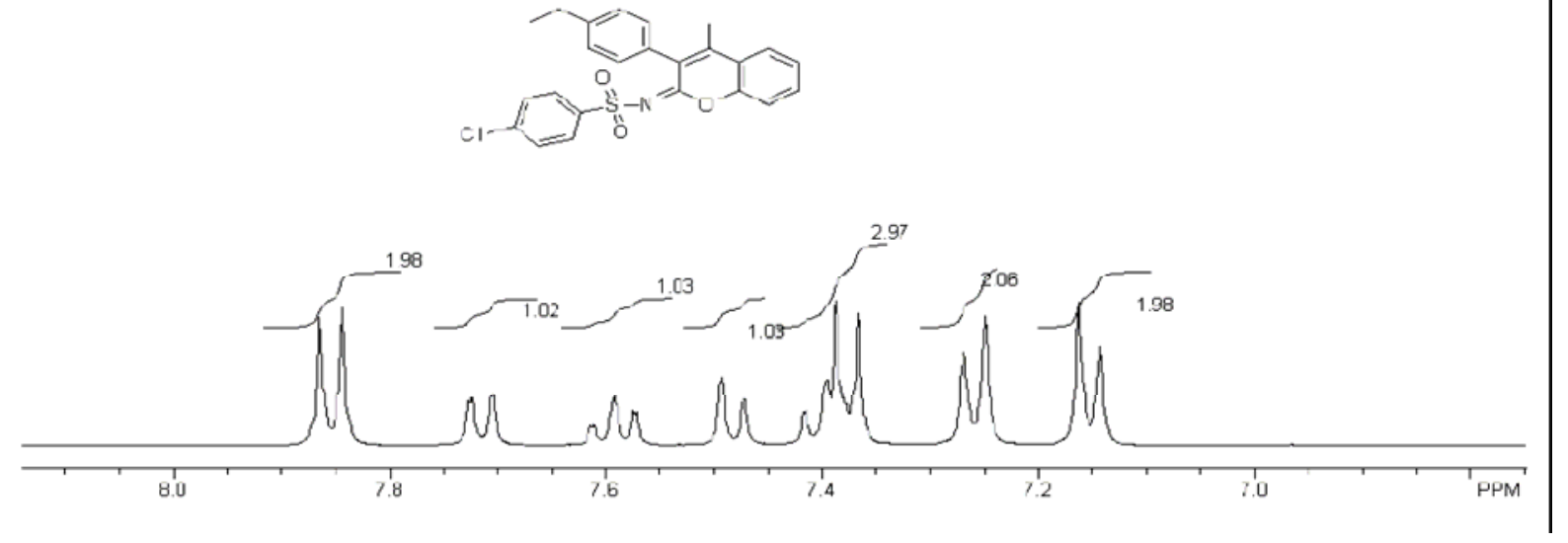


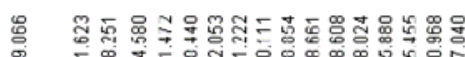

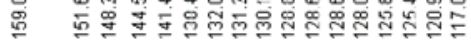

$1 \mid 1$ (1)

||
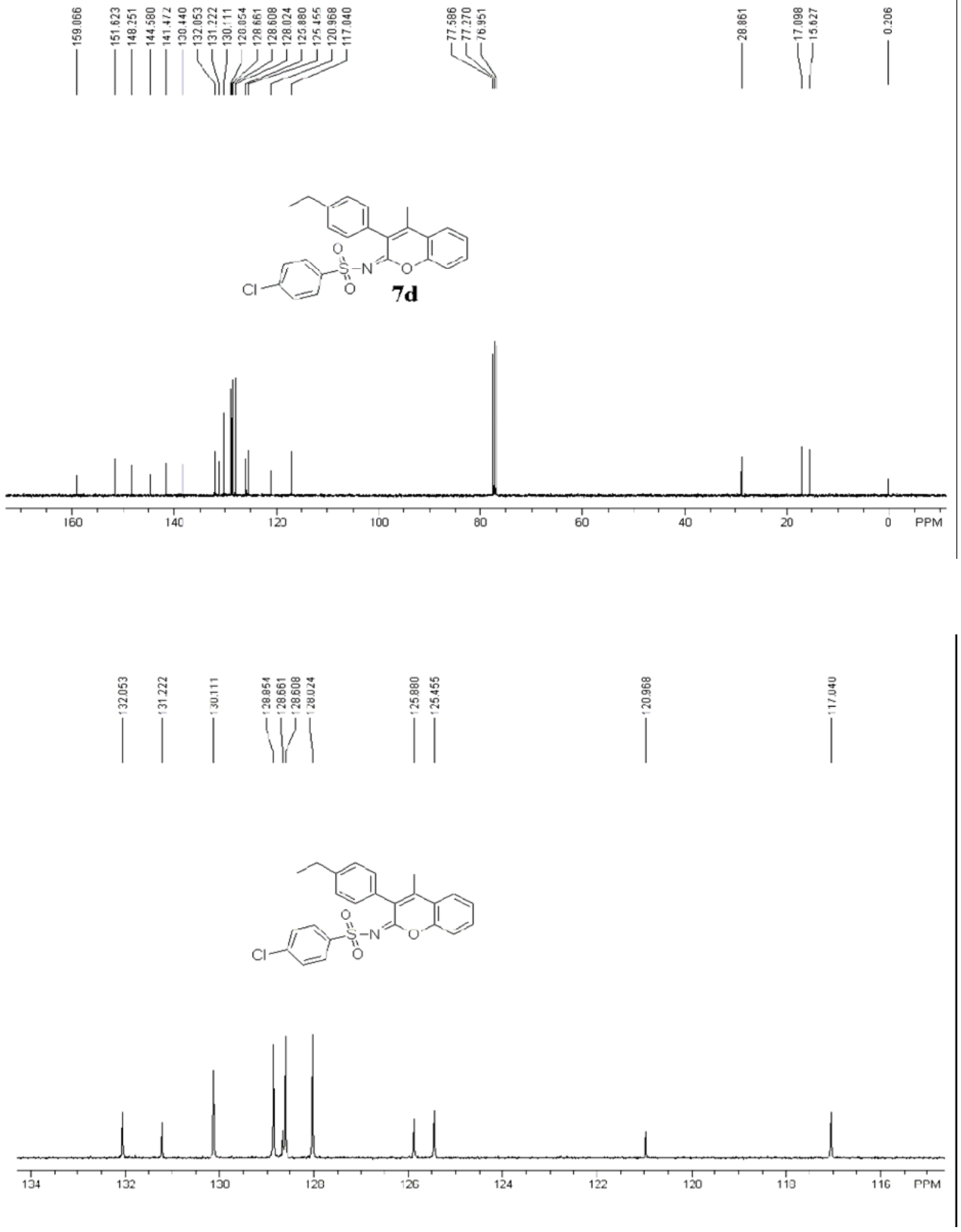


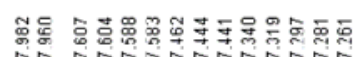

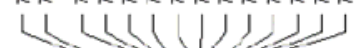

- vilif

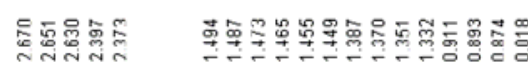

Un wivi
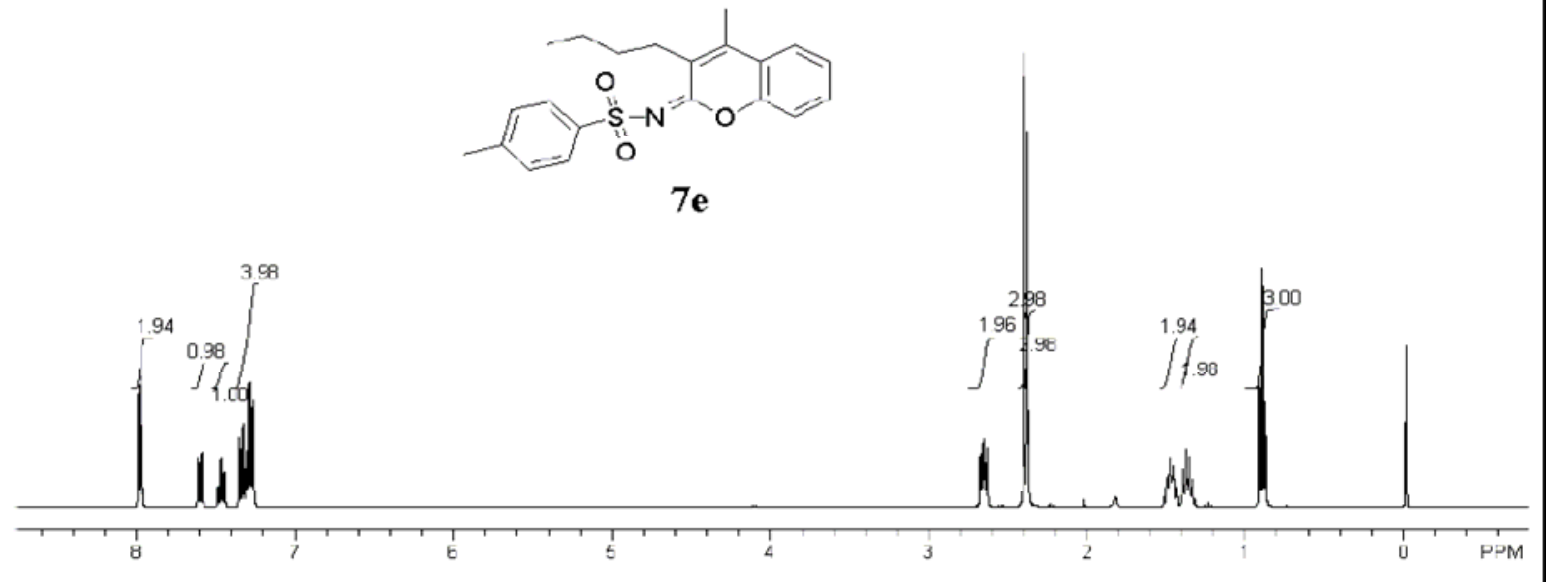

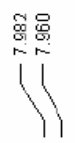

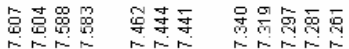

(1) S S S I
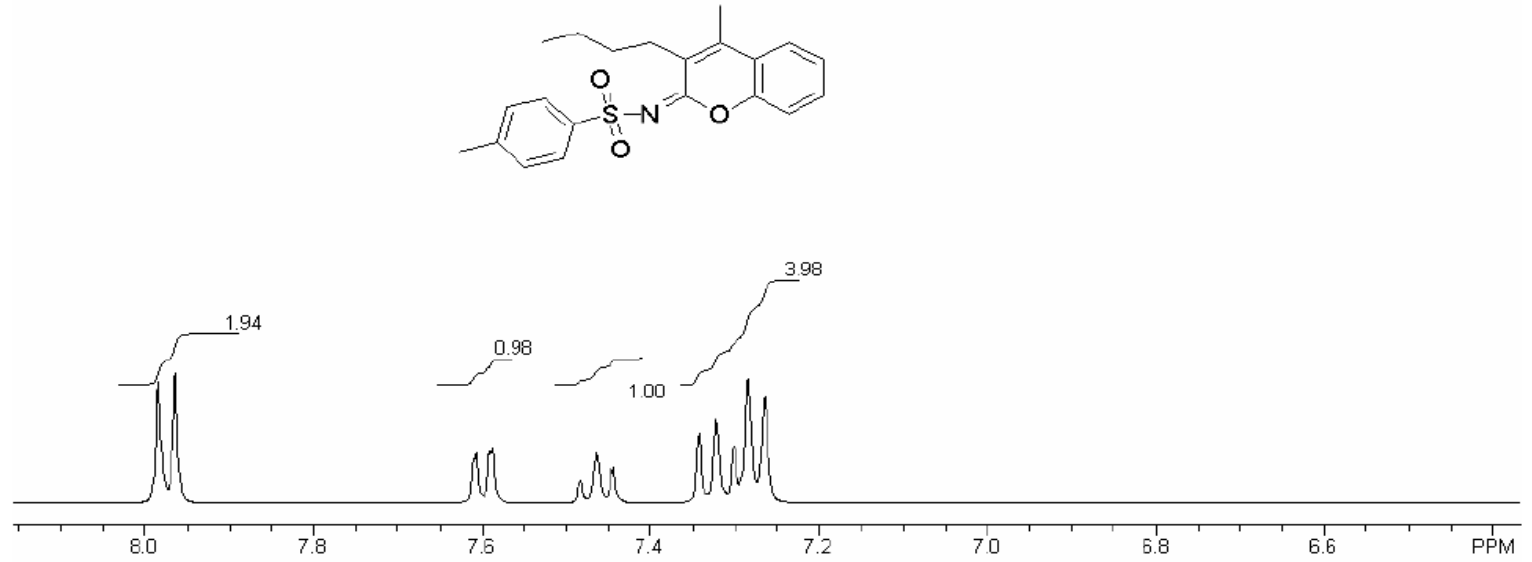

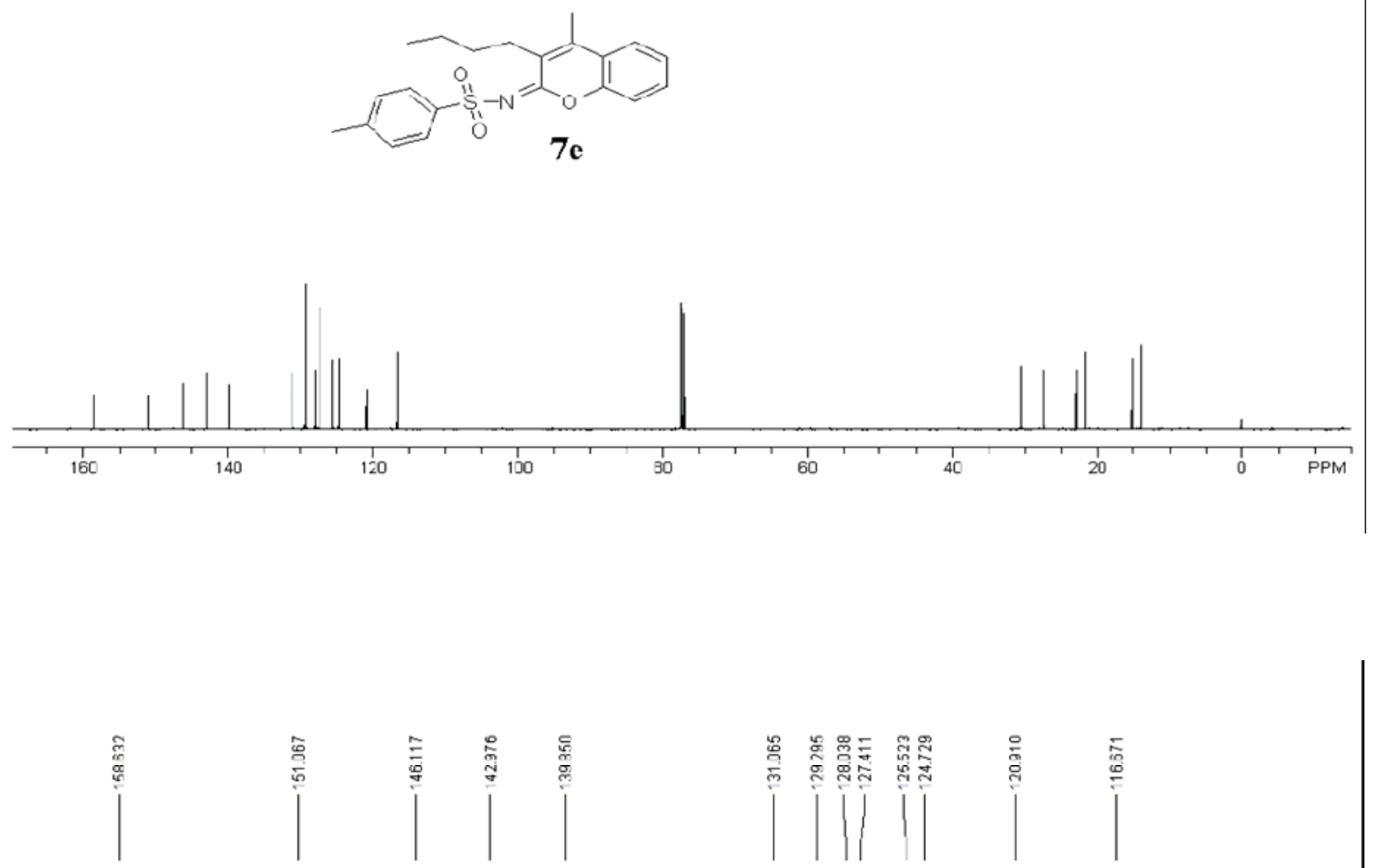<smiles>CCCCc1c(C)c2ccccc2o/c1=N/S(=O)(=O)c1ccc(C)cc1</smiles>

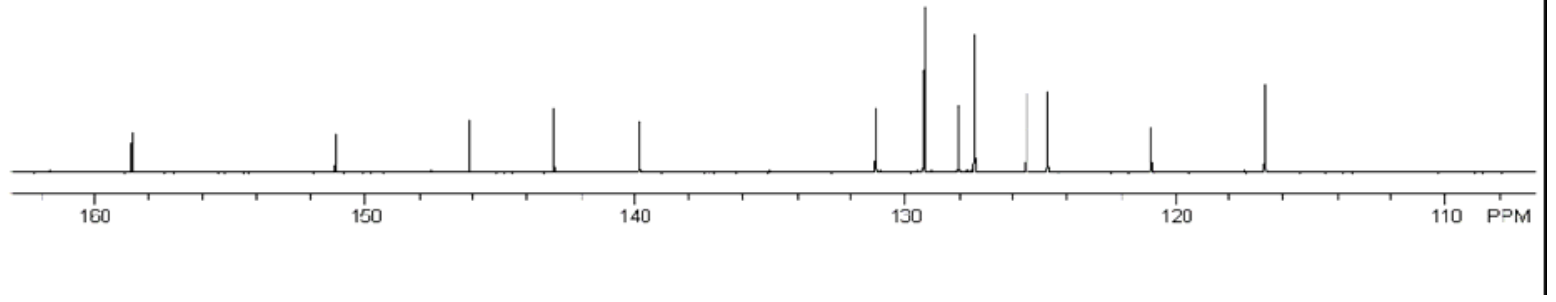




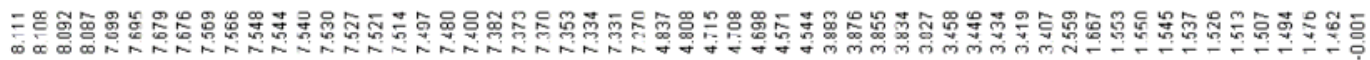

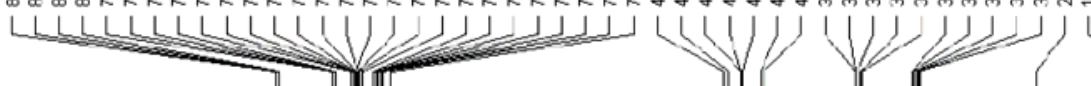

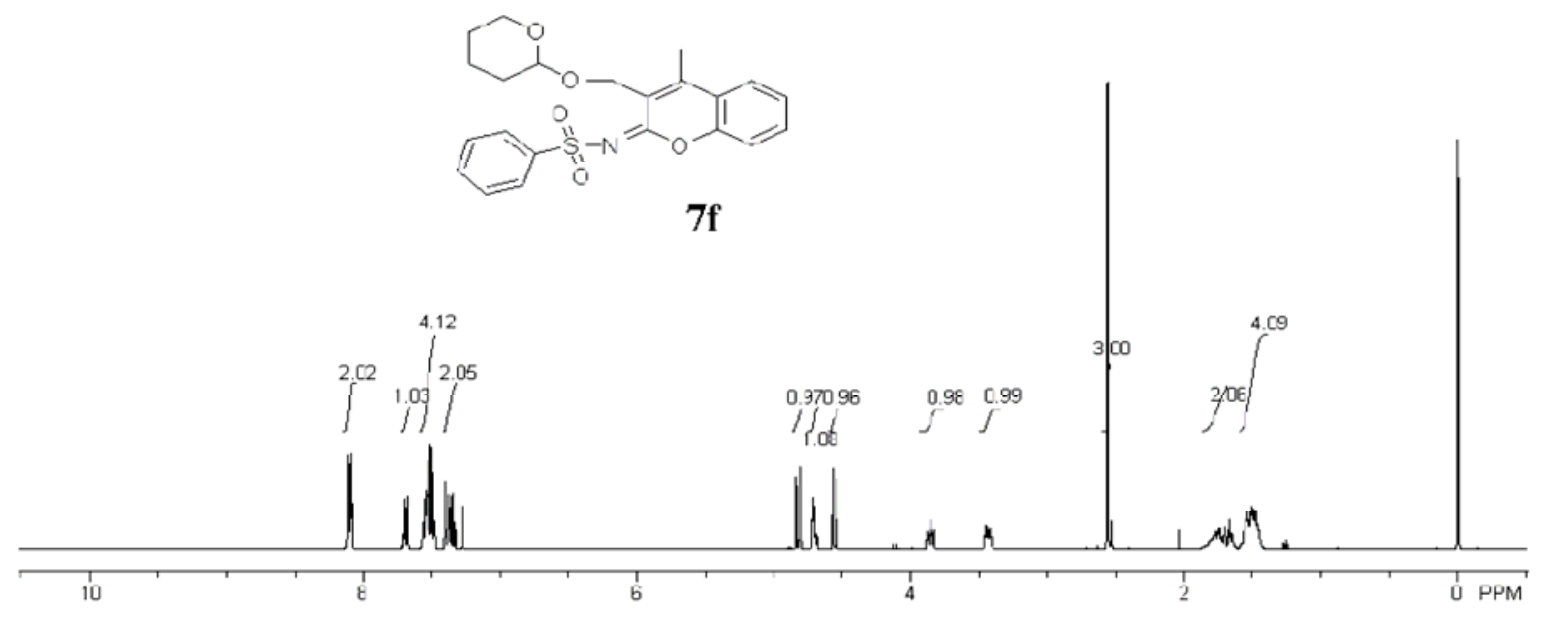

(1)
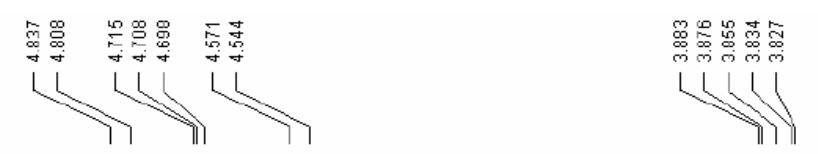

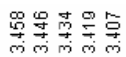

Li!
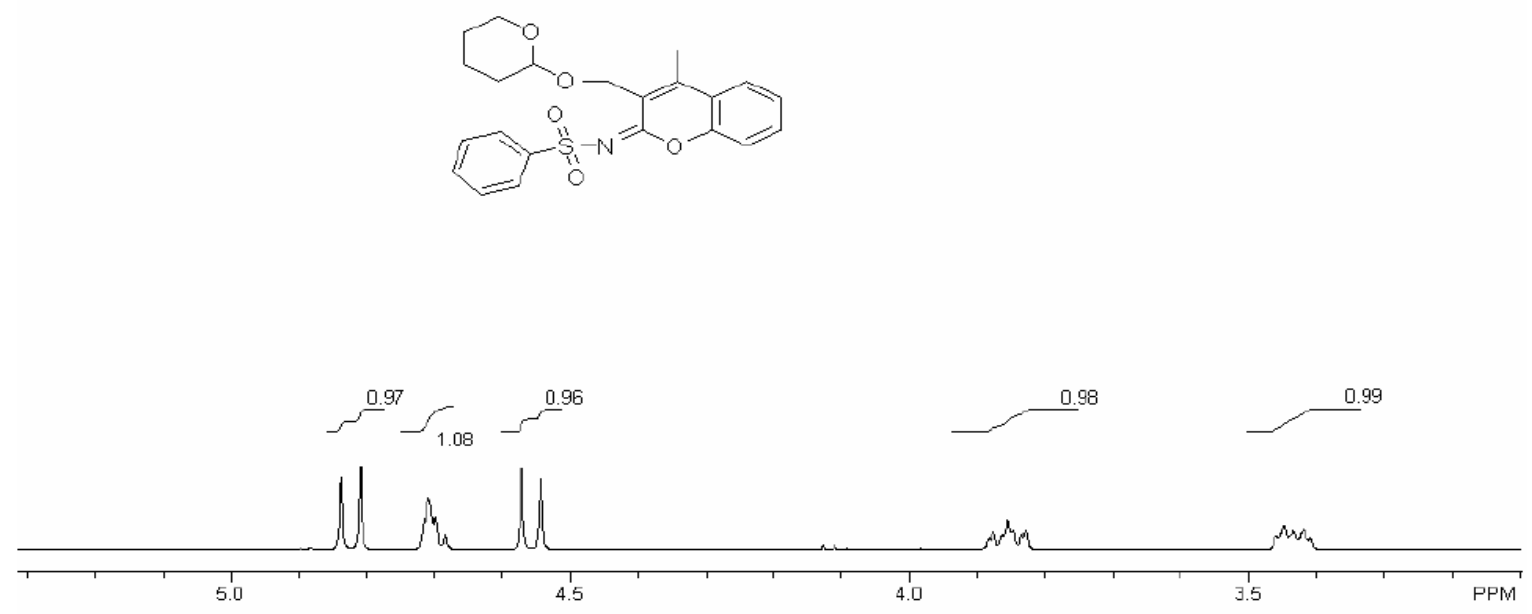

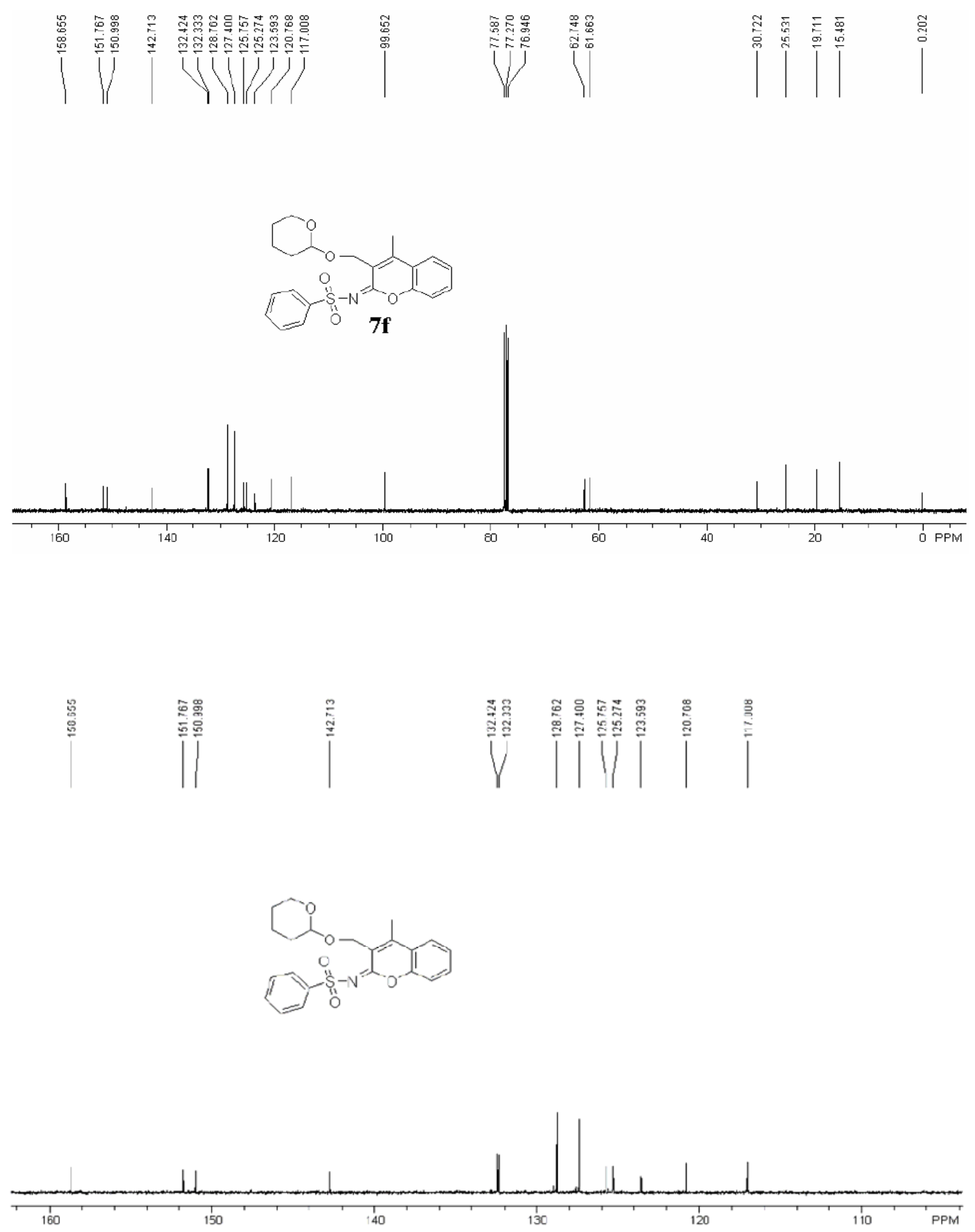